\title{
MIMO ACTIVE VIBRATION CONTROL OF MAGNETICALLY SUSPENDED FLYWHEELS FOR SATELLITE IPAC SERVICE
}

\author{
A Dissertation \\ by \\ JUNYOUNG PARK \\ Submitted to the Office of Graduate Studies of \\ Texas A\&M University \\ in partial fulfillment of the requirements for the degree of \\ DOCTOR OF PHILOSOPHY
}

May 2008

Major Subject: Mechanical Engineering 


\title{
MIMO ACTIVE VIBRATION CONTROL OF MAGNETICALLY SUSPENDED FLYWHEELS FOR SATELLITE IPAC SERVICE
}

\author{
A Dissertation \\ by \\ JUNYOUNG PARK \\ Submitted to the Office of Graduate Studies of \\ Texas A\&M University \\ in partial fulfillment of the requirements for the degree of \\ DOCTOR OF PHILOSOPHY
}

Approved by:

Chair of Committee, Alan B Palazzolo

Committee Members, Moo-Hyun Kim

Make McDermott

Alexander Parlos

Head of Department, Dennis O'Neal

May 2008

Major Subject: Mechanical Engineering 


\begin{abstract}
MIMO Active Vibration Control of Magnetically Suspended Flywheels for Satellite

IPAC Service. (May 2008)

Junyoung Park,

B.S., Kyung Hee University, South Korea;

M.S., University of Southern California

Chair of Advisory Committee: Dr. Alan B. Palazzolo
\end{abstract}

Theory and simulation results have demonstrated that four, variable speed flywheels could potentially provide the energy storage and attitude control functions of existing batteries and control moment gyros (CMGs) on a satellite. Past modeling and control algorithms were based on the assumption of rigidity in the flywheel's bearings and the satellite structure.

This dissertation provides simulation results and theory which eliminates this assumption utilizing control algorithms for active vibration control (AVC), flywheel shaft levitation and integrated power transfer and attitude control (IPAC) that are effective even with low stiffness active magnetic bearings (AMB), and flexible satellite appendages.

The flywheel AVC and levitation tasks are provided by a multi input multi output (MIMO) control law that enhances stability by reducing the dependence of the forward and backward gyroscopic poles with changes in flywheel speed.

The control law is shown to be effective even for (1) Large polar to transverse 
inertia ratios which increases the stored energy density while causing the poles to become more speed dependent and, (2) Low bandwidth controllers shaped to suppress high frequency noise. These two main tasks could be successfully achieved by MIMO (Gyroscopic) control algorithm, which is unique approach.

The vibration control mass (VCM) is designed to reduce the vibrations of flexible appendages of the satellite. During IPAC maneuver, the oscillation of flywheel spin speeds, torque motions and satellite appendages are significantly reduced compared without VCM. Several different properties are demonstrated to obtain optimal VCM.

Notch, band-pass and low-pass filters are implemented in the AMB system to reduce and cancel high frequency, dynamic bearing forces and motor torques due to flywheel mass imbalance. The transmitted forces and torques to satellite are considerably decreased in the present of both notch and band-pass filter stages.

Successful IPAC simulation results are presented with a 12 [\%] of initial attitude error, large polar to transverse inertia ratio $\left(I_{P} / I_{T}\right)$, structural flexibility and unbalance mass disturbance.

Two variable speed control moment gyros (VSCMGs) are utilized to demonstrate simultaneous attitude control and power transfer instead of using four standard pyramid configurations. Launching weights including payload and costs can be significantly reduced. 


\section{DEDICATION}

To my family 


\section{ACKNOWLEDGMENTS}

I would like to appreciate Dr. Palazzolo for his immeasurable considerations and concerns during my graduate study in the Vibration Control Electromachanical Lab at Texas A\&M University. I also would like to thank Dr. Make McDermott, Dr. Alexander Parlos and Dr. Moo-Hyun Kim for serving on my advisory committee.

I am grateful to my colleagues, Dr. Andrew Kenny, Randall Tucker, Clinton Johnson and Dr. Ming-Hsui Li, for their priceless help and friendship. I also express my sincere gratitude to NASA Center for Space Power at Texas A\&M and NASA Glenn for their helpful opinion and financial support.

I may not have finished this small achievement without encouragement, patient of my parents, wife and my children. I will love them forever. 


\section{TABLE OF CONTENTS}

Page

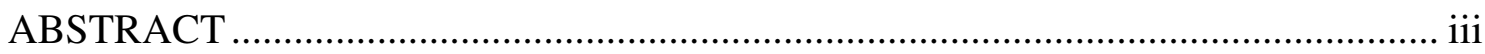

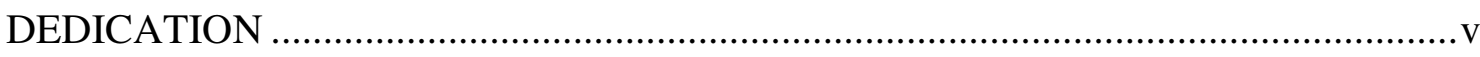

ACKNOWLEDGMENTS ..............................................................................

TABLE OF CONTENTS .............................................................................. vii

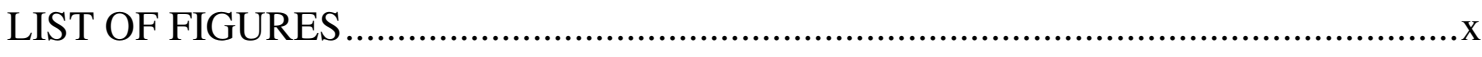

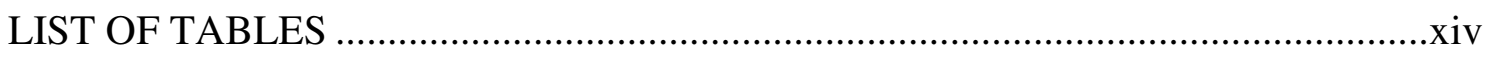

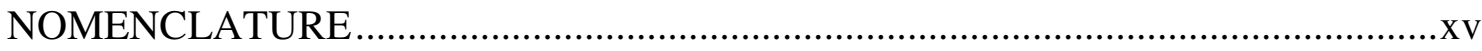

CHAPTER

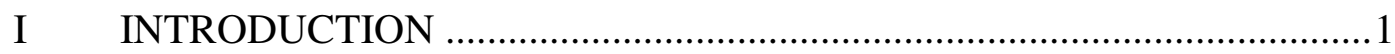

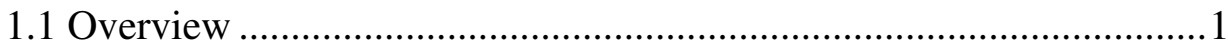

1.2 Literature Review ......................................................................... 5

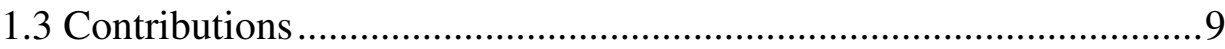

II SYSTEM MODEL AND STRUCTURAL DYNAMICS ........................ 10

2.1 Overview ............................................................................. 10

2.2 System Model Coordinates ........................................................ 11

2.3 Translational Motions of Flexible Flywheel and Appendage

Models ..................................................................................... 12

2.3.1 Translational Equation of Motion for a Rigid Flywheel Model [29].....................................................15

2.3.2 Translational Equation of Motion for a Pair of Neighboring Rigid Disks in the Flexible Flywheel and Appendage Models ...............................15

2.4 Rotational Motion of Flexible Flywheel and Appendage Models .... 16

2.4.1 Rotational Equation of Motion for a Rigid Flywheel Model 17

2.4.2 Rotational Equation of Motion for a Pair of Neighboring Rigid Disks in the Flexible 
Flywheel and Appendage Models ...................................17

2.5 Satellite Rotational and Translational Equations of Motions.............17

2.5.1 Rotational Motion (No External Torques) .....................18

2.5.2 Translation Motion (No External Forces) ......................18

III SATELLITE IPAC MANEUVER AND FEEDBACK CONTROL ............19

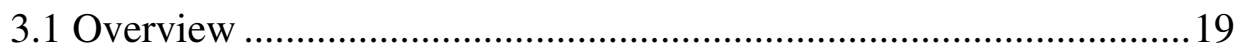

3.2 Satellite Reference Motion Design …………………………….....20

3.3 Flywheel Speed Control for IPAC ……………….........................22

3.3.1 IPAC Control Law......................................................22

3.3.2 Flywheel Motor Control Gain Selection .......................25

3.3.3 Torque Distributions and Power Tracking ……….........27

IV MAGNETIC BEARING SUSPENSION SYSTEM WITH MIMO

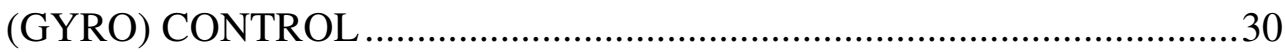

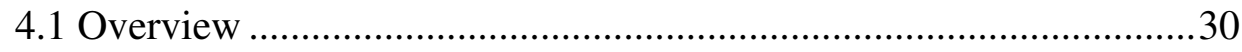

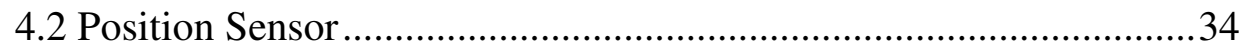

4.2.1 Voltage and Displacement Error at Position Sensor .....34

4.2.2 Motion Coordinate Transformation.................................35

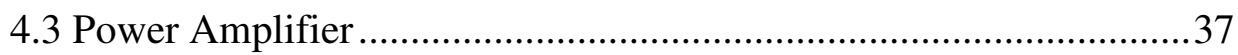

4.3.1 Control Currents ..........................................................

4.4 Magnetic Bearing - Actuator ……………………...............................

4.4.1 Control Currents Forces ...............................................41

4.4.2 Transformation Matrix …………………………….......42

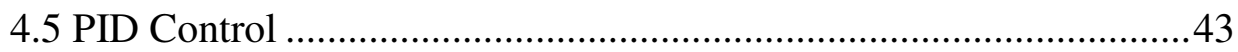

4.5.1 PD Transfer Function with Unity Gain ...........................43

4.5.2 PD Gain Stage for MIMO (GYRO) Control ..................44

4.6 Output Coordinate Transformation Stage ............................................46

4.7 Effective AMB Stiffness, Damping and Gyro Cancellation

Torque Coefficients .............................................................................. 48

$\mathrm{V}$ VIBRATION CONTROL OF FLEXIBLE APPENDAGES AND FLYWHEEL UNBALANCE ISOLATION ..............................................51

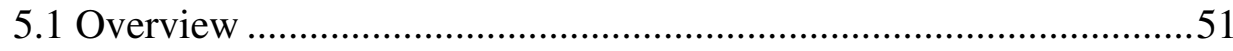

5.2 Vibration Control Mass (VCM) to Suppress the Oscillation of the Satellite's Flexible Appendages ...............................................51

5.3 Flywheel Unbalance Isolation ..........................................................54 
CHAPTER Page

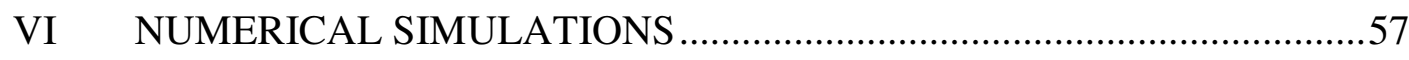

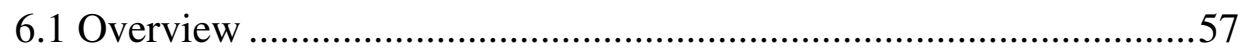

6.2 Model Description...................................................................59

6.3 Validation of Finite Element Model for Satellite Flexibility ...........62

6.4 Satellite Responses Including MB Suspension and Flexibility.........67

6.5 Comparison of SISO and MIMO AMB Suspension Control.............70

6.6 VCM Effects on Flywheel and Flexible Appendages Motions......... 74

6.6.1 VCM Effects on Flywheel Power Charging Case .........75

6.6.2 VCM Effects on Flywheel Power Delivery Case..........79

6.6.3 VCM Effects on Flexible Appendages Motion ..............84

6.7 Isolation of the Satellite from the Flywheel's Mass Imbalance

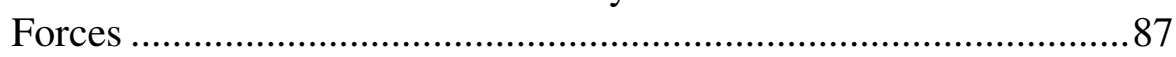

VII IPAC WITH TWO VARIABLE SPEED CONTROL MOMENT

GYROS ........................................................................................

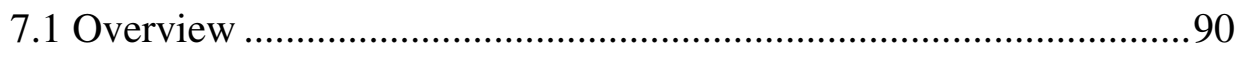

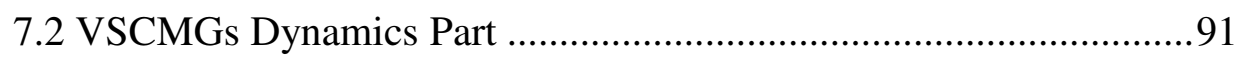

7.3 VSCMGs Control Law Part .......................................................95

7.4 VSCMGs Torque Distributions Part ..............................................97

7.5 VSCMGs Simulation Results .......................................................99

7.5.1 IPAC Simulation Results with Two VSCMGs ...........101

7.5.2 IPAC Simulation Results with Four VSCMGs ...........106

VIII CONCLUSION AND FUTURE WORK ......................................... 110

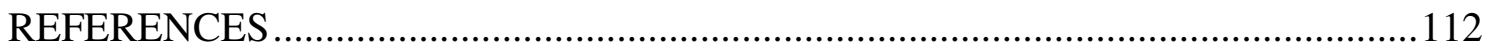

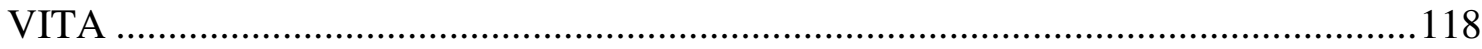




\section{LIST OF FIGURES}

FIGURE

Page

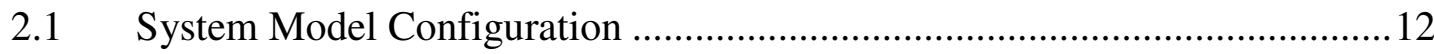

2.2 Inertial, Satellite, Housing, Flywheel and Appendage Coordinate Systems .. 13

2.3 Nodal Degrees of Freedom for a 3D Beam Type Finite Element .................. 14

3.1 Root-Locus Plot of the Decoupled, Linearized Error Dynamics ..................27

4.1 MB Suspension System Feedback Control Diagram for MIMO(GYRO) .....32

4.2 Flywheel System with Magnetic Bearing(MB) Suspension ..........................32

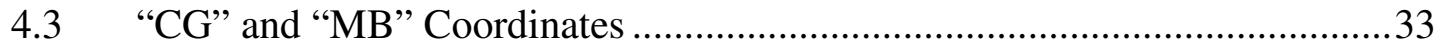

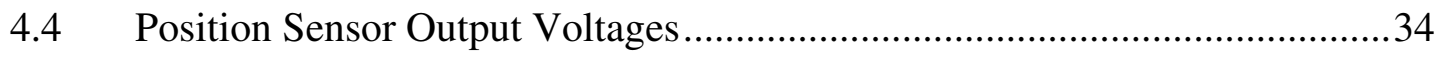

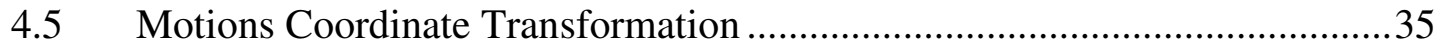

4.6 First Order Transfer Function of PWM.......................................................

4.7 C-core Electromagnet and Rotor Lamination Stack..................................39

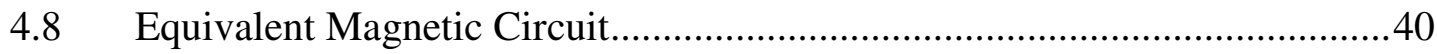

4.9 Force- Moment Transformation Diagram ................................................42

4.10 Unity Gain PD Transfer Function Stage ...............................................44

4.11 MIMO-GYRO PD Gain Diagram .......................................................... 45

4.12 Diagrams for Output Coordinate Transformation .......................................46

5.1 Vibration Control Mass (VCM) Model .......................................................52

5.2 Characteristics of Band-Pass and Notch Filters ........................................55

5.3 AMB Control to Attenuate the Forces at the Spin Frequency.......................55 
FIGURE Page

6.1 IPAC System Feedback Control Loop ……………......................................58

6.2 Tetrahedral Array of Flywheels Attached to the Satellite .................................60

6.3 Flexible Appendage Model Consisting of Beam Type Elements ...................60

6.4 Long Rigid-Rotor Model with Coordinate System ........................................65

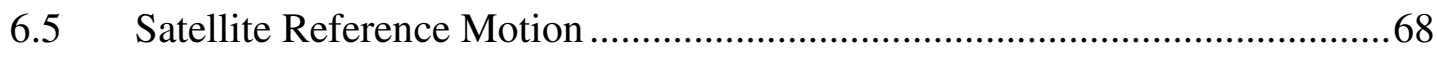

6.6 Satellite Motions Including Flexibility and MB Suspension System..............69

6.7 Satellite Error Motions ………………………………................................69

6.8 Torques Applied to the Satellite ……………….............................................70

6.9 Displacements of Flywheels at Sensor Position with SISO Control ...............71

6.10 Displacements of Flywheels at Sensor Position with MIMO Control ............72

6.11 MB Forces at Each Module (SISO Control) ……….....................................72

6.12 MB Forces at Each Module (MIMO Control) …………..................................73

6.13 Coil Voltage with SISO Control..................................................................73

6.14 Coil Voltage with MIMO Control .................................................................74

6.15 Flywheel Motions without VCM for Power Charging Case .............................75

6.16 Flywheel Motions with VCM (1.35[kg]) for Power Charging Case ...............76

6.17 Attitude Control Torque and Power Charging Torque without VCM ............76

6.18 Attitude Control Torque and Power Charging Torque with VCM..................77

6.19 Power Charging Response without VCM ……………………....................77

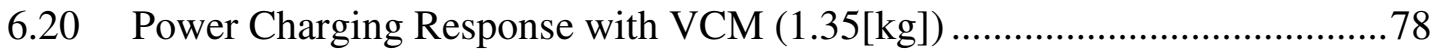

6.21 Magnified Power Transfer (Charging) without VCM....................................78 
FIGURE Page

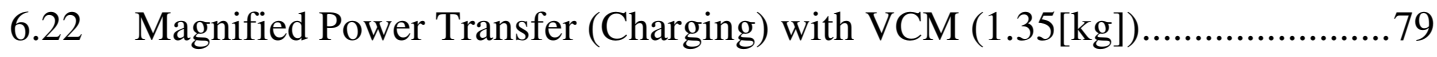

6.23 Flywheel Motions without VCM for Power Delivery Case ......................... 80

6.24 Flywheel Motions with VCM (1.35[kg]) for Power Delivery Case..............81

6.25 Attitude Control Torque and Power Delivery Torque without VCM ...........81

6.26 Attitude Control Torque and Power Delivery Torque with VCM ................82

6.27 Power Delivery Response without VCM ............................................. 82

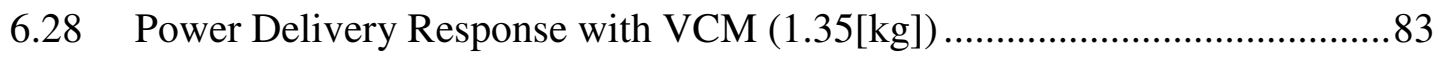

6.29 Magnified Power Transfer (Delivery) without VCM..................................83

6.30 Magnified Power Transfer (Delivery) with VCM $(1.35[\mathrm{~kg}])$......................84

6.31 Vibration along Satellite Appendage During IPAC without VCM...............85

6.32 Vibration along Satellite Appendage During IPAC with VCM $(1.35[\mathrm{~kg}]) \ldots .85$

6.33 Maximum Power Ripple VS Vibration Control Mass ...............................86

6.34 Maximum Relative Stroke of Appendage VS Vibration Control Mass ........86

6.35 Transmitted Forces and Torques without Notch and Band Pass Filter ..........88

6.36 Transmitted Forces and Motor Torques with Notch Filter............................88

6.37 Transmitted Forces and Motor Torques with Notch and Band Pass Filter ....89

6.38 Transmitted Forces and Motor Torques with All Filter Stages ......................89

7.1 One VSCMG Coordinate System....................................................... 91

7.2 Two VSCMGs IPAC Service Configuration ......................................... 100

7.3 Satellite Motions with Two VSCMGs ................................................... 103

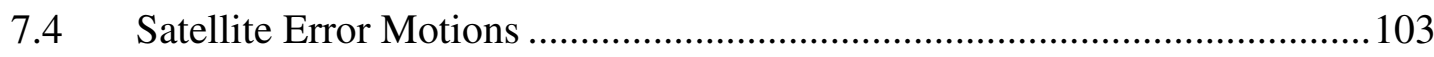


FIGURE Page

7.5 Proximity Scalar and Weights Factor .................................................. 104

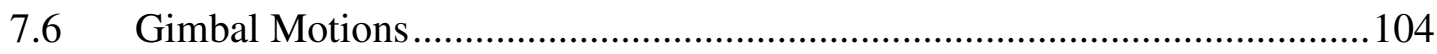

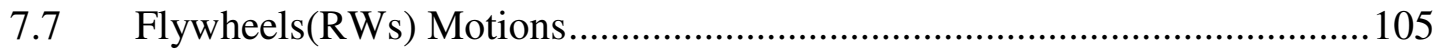

7.8 Power Transfer during Attitude Control............................................... 105

7.9 Satellite Motions with Four VSCMGs .................................................. 107

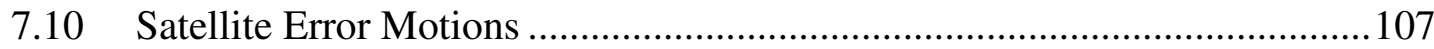

7.11 Proximity Scalar and Weights Factor ............................................... 108

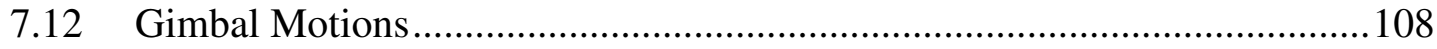

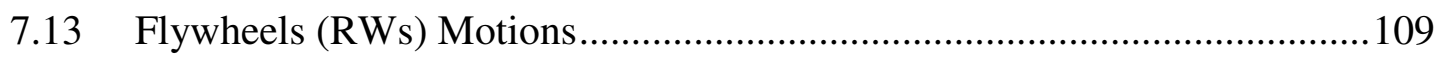

7.14 Power Transfer during Attitude Control..................................................... 


\section{LIST OF TABLES}

TABLE $\quad$ Page

5.1 VCM Damping Ratio VS VCM Attachment Stiffness and Damping ..........54

6.1 Model Parameter Values ..............................................................61

6.2 AMB Parameter Values.....................................................................62

6.3 AMB and Flywheel Motor Control Gains .............................................62

6.4 Parameters of Long Rigid-Rotor Model.................................................66

6.5 Comparison of Natural Frequencies between Analytic and FE Model ..........66

7.1 VSCMG Simulation Parameters............................................................ 101 


\section{NOMENCLATURE}

$$
\begin{aligned}
& A^{e}=\text { Cross section area of beam element } \\
& A_{p}=\text { Cross section area of the magnetic pole } \\
& c \quad=\text { Nominal air gap } \\
& E^{e} \quad=\text { Young's modulus of beam element } \\
& f_{f} \quad=\text { Flux de-rate factor due to fringing } \\
& F_{f} \quad=\text { Magnetic Bearing reaction forces acting on the flywheel } \\
& G^{e}=\text { Elastic shear modulus of beam element } \\
& i=\text { Total current (bias plus control) } \\
& i_{b} \quad=\text { Biased current } \\
& i_{c}=\text { Control current } \\
& I_{f} \quad=\text { Rotational inertia for the flywheel } \\
& I_{P} \quad=\text { Flywheel polar moment of inertia } \\
& I_{s} \quad=\text { Satellite inertia including flywheel housings } \\
& I_{T} \quad=\text { Flywheel transverse moment of inertia } \\
& I_{x i}^{e}=\text { Area moment of inertia of beam element } \\
& J^{e}=\text { Torsion constant of beam element } \\
& K_{c u r}=\text { Linearized current stiffness }
\end{aligned}
$$




$$
\begin{array}{ll}
K_{p o s} & =\text { Linearized position stiffness } \\
l & =\text { Euler's principle axis } \\
l_{A M} & =\text { Distance between Flywheel CG and MB }(A) \\
l_{A S} & =\text { Distance between Flywheel CG and Sensor }(A) \\
l_{B M} & =\text { Distance between Flywheel CG and MB }(B) \\
l_{B S} & =\text { Distance between Flywheel CG and Sensor }(B) \\
L_{e} & =\text { Beam element length of beam element } \\
m & =\text { The number of appendage disks in one appendage finite element model } \\
m_{f} & =\text { Flywheel mass } \\
M_{s} & =\text { Satellite mass including flywheel housings } \\
N & =\text { Number of coil turns } \\
P & =\text { Required power } \\
R_{a} & =\text { Offset distance between flywheel housing mass center and satellite CG } \\
R_{f} & =\text { Offset distance between flywheel housing mass center and satellite CG } \\
t_{f} & =\text { Satellite maneuver time (60[sec]) } \\
T_{f} & =\text { Torque applied to the flywheel } \\
T_{m b} & =\text { Flywheel magnetic bearing torque } \\
T_{m t} & =\text { Flywheel motor torque } \\
\Delta t & =\alpha \cdot t_{f} \\
V_{Y A} & =\text { Sensor voltage at position } A \text { in the } Y \text { direction } \\
T^{\prime} &
\end{array}
$$


$V_{Y A}^{S, T}=$ Target sensor voltage at position $A$ in the $Y$ direction

$V_{Y B}^{S}=$ Sensor voltage at position $B$ in the $Y$ direction

$V_{Y B}^{S, T}=$ Target sensor voltage at position $B$ in the $Y$ direction

$V_{Z A}^{S}=$ Sensor voltage at position $A$ in the $Z$ direction

$V_{Z A}^{S, T}=$ Target sensor voltage at position $A$ in the $Z$ direction

$V_{Z B}^{S}=$ Sensor voltage at position $B$ in the $Z$ direction

$V_{Z B}^{S, T}=$ Target sensor voltage at position $B$ in the $Z$ direction

$x=$ Displacement of flywheel relative to flywheel housing

$x_{c}=$ Rotor displacement from the center of the bearing

$(\dot{x})=$ An over-dot with parenthesis and subscript denotes the differentiation with respect to time as viewed in the frame indicated by the subscript

$X=$ Displacement of the satellite mass center relative to the inertial frame

$X_{f / s}^{h}=$ Displacement of flywheel mass center relative to the satellite in the flywheel housing frame

$\dot{X}_{f / s}^{h}=$ Flywheel CG velocity relative to the satellite in the flywheel housing frame

$\dot{X}_{f / n}^{h}=$ Flywheel CG velocity relative to the inertial frame in the flywheel housing frame

$y=$ Displacement of appendage relative to appendage reference frame

$Y_{A}^{S}=$ Displacement of $A$ at sensor position in the $Y$ direction

$Y_{A}^{S, T}=$ Target displacement of $A$ at sensor position in the $Y$ direction 
$Y_{B}^{S} \quad=$ Displacement of $B$ at sensor position in the $Y$ direction

$Y_{B}^{S, T}=$ Target displacement of $B$ at sensor position in the $Y$ direction

$Z_{A}^{S} \quad=$ Displacement of $A$ at sensor position in the $Z$ direction

$Z_{A}^{S, T}=$ Target displacement of $A$ at sensor position in the $Z$ direction

$Z_{B}^{S} \quad=$ Displacement of $B$ at sensor position in the $Z$ direction

$Z_{B}^{S, T}=$ Target displacement of $B$ at sensor position in the $Z$ direction

$[a b]=$ Direction cosine matrix between coordinates $a$ and $b$

$\sum \quad=$ The number of flywheel or appendage module. In the work, flywheel module is 4 and appendage is 2

$\sum_{j=1}^{m}=$ The number of disks in the each appendage $(m=5)$

$\underline{\text { Greek }}$

$\alpha=$ Controls the sharpness of the function $f$

$\xi \quad=$ Damping ratio

$\zeta=$ Sensor gain

$\zeta_{c l y}=$ Cylindrical mode damping ratio

$\zeta_{c o n}=$ Conical mode damping ratio

$\mu_{0} \quad=$ Permeability of free space

$\Omega_{f} \quad=$ Flywheel angular velocity relative to the flywheel housing in the flywheel frame 
$\Omega_{s}=$ Satellite angular velocity in the satellite frame

$\omega=$ Natural frequency

$\omega_{c o n}=$ Conical mode frequency

$\omega_{c l y}=$ Cylindrical mode frequency

$\omega_{f}=$ Flywheel angular velocity relative to the inertial frame

$\omega_{\text {spin }}=$ Flywheel spin frequency

$\omega_{a / b}=$ Angular velocity of $a$ relative to $b$

$\widetilde{\omega}=\left[\begin{array}{ccc}0 & -\omega_{3} & \omega_{2} \\ \omega_{3} & 0 & -\omega_{1} \\ -\omega_{2} & \omega_{1} & 0\end{array}\right]$ 


\section{CHAPTER I}

\section{INTRODUCTION}

\subsection{Overview}

Satellite weight and cost reduction goals may benefit from Satellite Integrated Power and Attitude Control (IPAC). This will be accomplished by replacing the present energy storage system (electrochemical batteries) and attitude control torque actuator (control moment gyros) with an array of 4 high performance and speed flywheels [1]. Successful implementation of IPAC requires a control approach that uncouples the attitude control and power transfer functions so as to avoid unplanned motion actuation due to power transfer and unplanned power transfer due to satellite motion actuation. This separation of functions can be realized by utilizing attitude control torques obtained from the range space of underdetermined system and power transfer torques from the orthogonal null space $[1,2]$.

The prior IPAC literatures focused on control algorithm development which assumed that the satellite structure (no flexible appendage model), flywheel shafts and flywheel bearings were all rigid and that the flywheels were perfectly mass balanced to ignore the mass imbalance sinusoidal disturbance which occurs at the spin speeds of the flywheels. Even though, the flywheels are manufactured delicately, the imbalance still exists on it. This approach further simplified the problem by assuming that the motions

This dissertation follows the style and format of the ASME Journal of Dynamic Systems, Measurement and Control. 
of each flywheel could be adequately modeled with a single degree of freedom per flywheel (executing only spin motion).

The high speed, longevity, contamination and loss requirements for these flywheels mandate that magnetic bearings (MB) be utilized for suspension of the spinning rotor. The magnetic bearings have many advantages over the traditional bearings such as no contact between the shaft and stator, no lubrication, high spin speed operation, and adjustable equivalent damping and stiffness, which are functions of controller parameters [3]. The stiffness and damping of the magnetic bearings may be conveniently adjusted through gain changes in their feedback control electronics.

In contrast to the assumptions employed in prior IPAC publications, the bearing stiffness is intentionally set at a low value to yield high frequency force isolation between the satellite and the spinning shafts. Also, the transmitted forces and torques could be significantly diminished by employing several filter stages in the magnetic bearing feedback control loop. The versatility and low loss benefits of the magnetic bearings are gained only by incorporating sophisticated control algorithms to reject shaft and satellite borne disturbances while maintaining stable control.

The MB control task is made complicated by the presence of speed dependent poles that result from gyroscopic moments of the spinning, vibrating shafts. The effect of speed dependent poles is magnified as an increased energy density demand on the flywheel is met by increasing the ratio $\left(I_{P} / I_{T}\right)$, of the polar to transverse mass moments of inertia of the spinning rotors. These poles typically bifurcate from their zero speed values into a forward and a backward whirling pole pair, where the direction of vibration 
whirl is forward (backward) for whirl in the direction (opposite) of spin.

The rigid body gyroscopic poles asymptotically approach $0[\mathrm{~Hz}]$ (backward pole) and $\left(I_{P} / I_{T}\right)$ times spin frequency (forward pole) producing a very low frequency pole and a very high frequency pole for $I_{P} / I_{T}>1$. The strength of gyroscopic moment depends on the ratio $\left(I_{P} / I_{T}\right)$, which becomes larger as the rotor is pancake shape rather than cylindrical one. This complicates the control task since increased active damping (derivative gain) is ineffective at low frequencies and causes noise amplification at high frequency. Also, the high frequency pole (forward conical mode) results in voltage saturation in the power amplifier. It with frequency increases with spin speed so requiring phase lead, more derivative gain, larger currents at high speed, and finally the coil voltage in the power amplifier would be saturated.

Effective MB control then requires a shift in strategy from providing phase lead by derivative gain changes to canceling gyroscopic torques utilizing a multiple inputmultiple output (MIMO) control approach. The gyroscopic torque cancellation strategy requires that control "pitch" torques be applied to the rotor in one plane that are proportional to the shaft " $y a w$ " angular motions in the quadrature plane. Hence the shaft motions that are sensed near to the MB's could be converted into coordinates that approximately describe the translation of the shaft's mass center and rigid body rotations about it ("CG" coordinates). These form the inputs to the MIMO control algorithm. The outputs of the control algorithm are CG force and torque commands that are converted to force commands at the MB's in both planes. The relationship between "CG" and "MB" coordinates is presented in the Chapter IV. 
From this discussion it is apparent that significant technical detail, as presented in this paper, is required to apply the general algorithms for IPAC that appear in the literature to actual satellite systems.

The demand of maintaining a jitter free environment on the spacecraft inspired a novel contribution for utilizing band-pass filters that track flywheel spin speed to assist in canceling shaking forces caused by the imbalanced spinning flywheel shafts at their spin frequencies. The source of this force is that all magnetic bearings possess a passive negative stiffness making them open-loop unstable. The orbit (vibration) motion of the shaft section in the magnetic bearing combines with the negative stiffness to produce a shaking force (transmitted force) on the satellite at the shaft spin frequency. The tracked vibration component is inverted and routed through a gain stage to produce a signal for nulling the negative stiffness induced shaking forces.

The flexible appendage models are utilized to introduce low frequency modes into the plant as suggested to the authors by satellite design engineers. These may represent solar panels or other mission related equipment. For sake of simplicity, the appendages are modeled as uniform beams with very low values of equivalent Young's modulus to produce low frequency and lightly damped modes. Vibrations of the appendages during an attitude control and power tracking cause low frequency, small amplitude oscillations in the power transferred into or out of the flywheel array. These vibrations and the ensuing oscillations are significantly attenuated by attachment of a "Vibration Control Mass (VCM)" at the free end of both appendages. The optimal stiffness and damping of the VCM are obtained with a simplified assumed modes model of the appendages. 
The following sections attempt to answer questions posed by satellite design engineers related to implementing IPAC: (1) Is satellite IPAC effective with structural flexibility included in the bearings, flywheel shaft and appendages, (2) Is it possible to stabilize all eigenvalues related to the flywheel-MB system in the IPAC system of (1), and (3) Can low frequency appendage mode interference of IPACS be passively suppressed.

Two variable speed control moment of gyros are presented to show simultaneous attitude control and power transfer functions without interfering each other. The dynamics and control laws of four standard pyramid configuration VSCMGs are developed in the literature [4] and simultaneous attitude control and power tracking are performed in [5] with four VSCMGs case. However, only two VSCMGs are utilized to demonstrate successful IPAC service in this research.

\subsection{Literature Review}

Utilizing flywheels for energy storage on satellites was suggested as early as 1961 in the Roes paper [6]. Sindlinger [7] and Brunet [8] discussed the advantages of the MB suspension of a flywheel for attitude control and energy storage. Flatly [9] employed a tetrahedral array of four momentum wheels to consider the issues associated with applying wheel control torques for simultaneous attitude control and energy storage.

Tsiotras [10] introduced a logarithmic term for a kinematical parameter in the Lyapunov function that makes the controller corresponding to this parameter become linear. Schaub et al [11] presented a nonlinear feedforward / feedback controller for a prototype for large three dimensional rotational satellite maneuver and the actual closed 
-loop controller and estimator matched very well with the dynamics predicted in the feedback gain selection. This strategy for choosing flywheel motor feedback gains in this paper was reference in the Chapter III and also Landmark-tracking spacecraft, Nearminimum time and near-minimum fuel reference control torques were utilized in the Chapter III. Tsiotras et al [1] presented a control law for an integrated power and attitude control system for a rigid satellite with momentum wheels/reaction wheels. Y. Kim [2] outlined implementation of IPAC for a rigid structural satellite with SISO magnetic bearing control system.

Okada et al [12] utilized a proportional, cross feedback control to stabilize a highspeed rotor supported on magnetic bearings. Ahrens et al [13] also verified that the cross-feedback control leads to better system performance and improved stability for a flywheel-AMB energy storage system with strong gyroscopic coupling moments. U. Na et al [14] presented algorithms for fault-tolerant control of heteropolar magnetic bearings. Raoul Herzog et al [15] proposed a generalized narrow-band notch filter which is inserted into the multivariable feedback without destabilizing the closed loop and has advantages in terms of runtime complexity and analytical verification of closed loop stability.

Sanjay P. Bhat et al [16] showed that a continuous dynamical system on a state space that has the structure of a vector bundle on a compact manifold possess no globally asymptotically stable equilibrium and they explained how attitude stabilizing controllers appearing in the literature lead to unwinding instead of global asymptotic stability. S. Parman and H. Koguchi [17] presented a three-dimensional rest-to-rest 
attitude control of a flexible spacecraft equipped with on-off reaction jets, utilizing finite elements for modeling of flexible solar panels and with a Lagrangian formulation for the equations of motion. They applied time-optimal and fuel-efficient input shapers to reduce the residual oscillation of its motion at several natural frequencies in order to get an expected pointing precision of the satellite.

Magnetic bearing supported flywheels for energy storage and satellite attitude systems $[2,18,19,20,21,22]$ appear in many publications, but without reference to MIMO (GYRO) control for higher polar to transverse inertia ratio stability or to utilization of band-pass filters for removing transmitted forces induced by the magnetic bearing position stiffness.

NASA related flywheel R\&D includes the pioneering work of Kirk et.al [23, 24, 25, 26] for improving energy density and for incorporating magnetic bearings. The work of Kenny, B. et. al. [27] integrated sensorless field oriented motor control which was successfully demonstrated at 60,000 rpm on a NASA flywheel. Christopher and Beach provide a comprehensive overview of the NASA Glenn flywheel program in [28].

The dynamics and control laws of four standard pyramid configuration variable speed control moment gyros are developed in [4]. Variable speed control moment gyros (VSCMGs) combines the advantages of the single gimbal control moment gyro (CMG) and reaction wheel (RW).It has rotation speed of RW and precession rate of CMG. Two different control steering laws (velocity based and acceleration based steering laws) are developed from the Lyapunov stability approach and compared simulation results with classical control moment gyro. The weighting matrix is utilized to obtain minimum norm 
solution (required torque) to achieve MRP attitude and angular velocity error regulation problem. The simultaneous attitude control and energy storage using four standard pyramid configuration VSCMGs were presented in [5]. In this literature, they used Euler parameters for attitude kinematics instead of Modified Rodrigues Parameter shown [4]. The attitude control torque and power tracking torque are obtained from the range space and the null space of dynamic matrix which is not $N$ by $N$ matrix and the velocity based steering control law was employ to achieve given tasks.

The present research demonstrates the effectiveness of a cross coupled, MIMO and AMB control approach for providing rotor-dynamic stability and vibration suppression during a simulated IPAC maneuver with flywheel bearing and satellite flexibility included in the model. The term cross coupled control signifies application of control torques in one plane, i.e.) $x-y$, due to angular motion in the quadrature plane, i.e.) $x-z$. This mimics the action of a gyroscopic torque which acts in one plane and is proportional the angular velocity in the quadrature plane. The MIMO control implements a strategy of gyroscopic torque cancellation, which reduces the dependence of the forward and backward conical mode poles on spin speed. This simplifies the control law by reducing its dependence on spin speed and reduces high frequency noise amplification by lowering the frequency of the forward conical mode, and in turn lowering the level of required derivative gain. 


\subsection{Contributions}

This presented research contains the following unique contributions;

(1) Significant extension of prior IPAC simulation implementations to include flexible shafts and satellite appendages along with MB suspended flywheel system. The flexibility of flywheel shafts and satellite appendages are considered for shaft higher mode and satellite solar panel model utilized by finite element analysis.

(2) Novel approach to isolate satellite imbalance forces from flywheels. Band pass filter stage is employed to diminish satellite transmitted forces due to residual forces created from flywheel relative displacement and position stiffness.

(3) Application of MIMO (GYRO) control algorithm for higher energy density flywheel (higher ratio of moment of inertia, $I_{P} / I_{T}$ ) including nonlinearity of MB suspension component such as power amplifier saturation.

(4) Two single gimbaled flywheels called as variable speed control moment gyro (VSCMG) are utilized to demonstrate simultaneous attitude control and power transfer functions without interfering each other instead of using four standard pyramid configurations presented in the literature [5]. 


\section{CHAPTER II}

\section{SYSTEM MODEL AND STRUCTURAL DYNAMICS*}

\subsection{Overview}

The Integrated Power and Attitude Control (IPAC) system model and structural dynamics including flexible flywheels and satellite appendages are developed in this chapter. Each coordinate system is described in the section 2.2 and dynamic differential equations are presented in the sections 2.3 thru 2.5. The translational and rotational motions of one rigid flywheel [2] are derived first to obtain flexible system model. Each finite element model comprised with $N$ rigid disks has same differential form of one rigid flywheel model.

Prior IPAC system control algorithm assumed that the flywheels are mounted on the satellite with infinite stiffness bearings, thus contributing only spin degree of freedom per flywheel (spin direction). However, in reality high speed flywheels will be supported by magnetic bearing (MB). The MB's compliance allows the flywheel to move with additional degree of freedoms relative to the satellite. Modeling of flywheel shaft flexibility adds even more degree of freedoms since its bending deformation provides relative motions in the shaft fixed frame [29]. Finite element analysis is utilized to model these flexible flywheel shafts and satellite appendages. The details are presented in the sections 2.3 thru 2.5 .

*Reprinted with permission from "MIMO Active Vibration Control of Magnetically Suspended Flywheels for Satellite IPAC Service," Park, J., 2007, Journal of Dynamic Systems, Measurement and Control, Accepted, Copyright [2008] by ASME. 


\subsection{System Model Coordinates}

The motions in the IPAC satellite model (Fig.2.1) are described based on the following coordinate systems:

(a) An inertially fixed coordinate system for the satellite's center of mass translations: $\left(\hat{n}_{1}, \hat{n}_{2}, \hat{n}_{3}\right)$

(b) Four satellite flywheel housing coordinates to indicate the very small relative motions of the flywheels with respect to the satellite at their housing (stator) locations: $\left(\hat{h}_{f, 1}, \hat{h}_{f, 2}, \hat{h}_{f, 3}\right)$

(c) Satellite body fixed coordinates for defining the satellite's angular velocity components: $\left(\hat{s}_{1}, \hat{s}_{2}, \hat{s}_{3}\right)$

(d) Four coordinate frames that precess, but do not spin, with the axisymmetric flywheels. The flywheel inertias are constant in these frames, thus the frames require only 2 instead of 3 angular coordinates to define the direction cosine matrix for each flywheel: $\left(\hat{f}_{1}, \hat{f}_{2}, \hat{f}_{3}\right)$

(e) Two satellite fixed coordinate frames are oriented along the undeformed appendages. Relative motion coordinates $\left(\hat{a}_{1}, \hat{a}_{2}, \hat{a}_{3}\right)$ define the small deflections of the appendages with respect to these coordinate axes: $\left(\hat{h}_{a, 1}, \hat{h}_{a, 2}, \hat{h}_{a, 3}\right)$

Only (a) and (c) coordinate systems are shown in the Fig.2.1 due to complexity but the rest of coordinate systems are depicted in the Fig.6.2 on pp.59 and Fig.6.3on pp.60, respectively. 


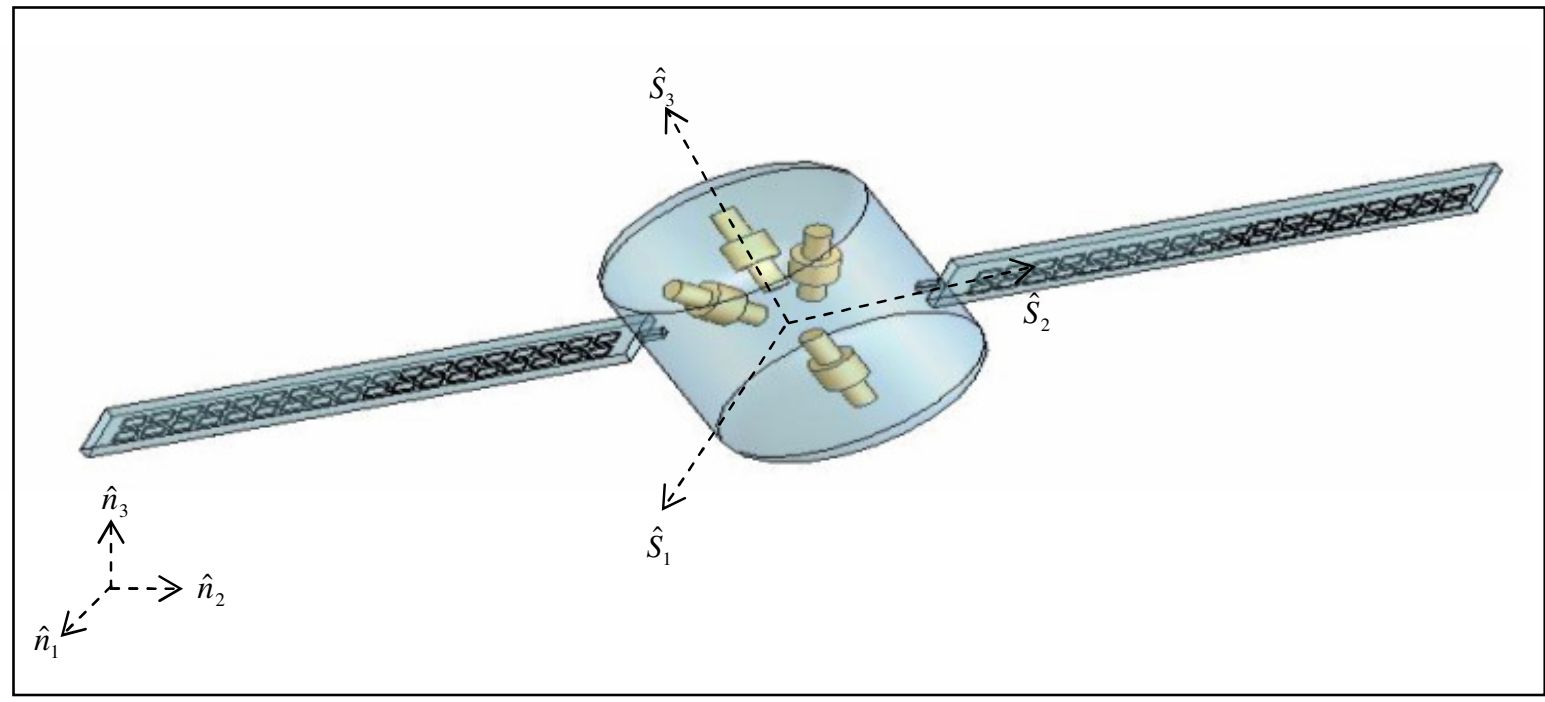

Fig. 2.1 System Model Configuration

\subsection{Translational Motions of Flexible Flywheel and Appendage Models}

The translational motion for one rigid flywheel module suspended magnetic bearing is obtained from the coordinate configuration shown in the Fig.2.2. The detailed explanation of each coordinate system is presented before. The relative flywheel and appendage displacements respect to flywheel housing and appendage reference frame are expressed as $x, y$ in the Fig.2.2. Based on this coordinate; the translational motion of a rigid flywheel in the flywheel housing frame [2,29] can be expressed in equation (2.5) and (2.6). Each flexible flywheel and appendage model can be divided into $N$ disks which have rigid body mass and inertia properties and is interconnected by flexible beam type finite elements. Each disk is modeled as executing 3D translational and rotational motion. The flexible flywheel and appendage for 2-noded, 6 degree of freedom per each node are modeled with 3D beam type finite elements as illustrated in the Fig.2.3. 


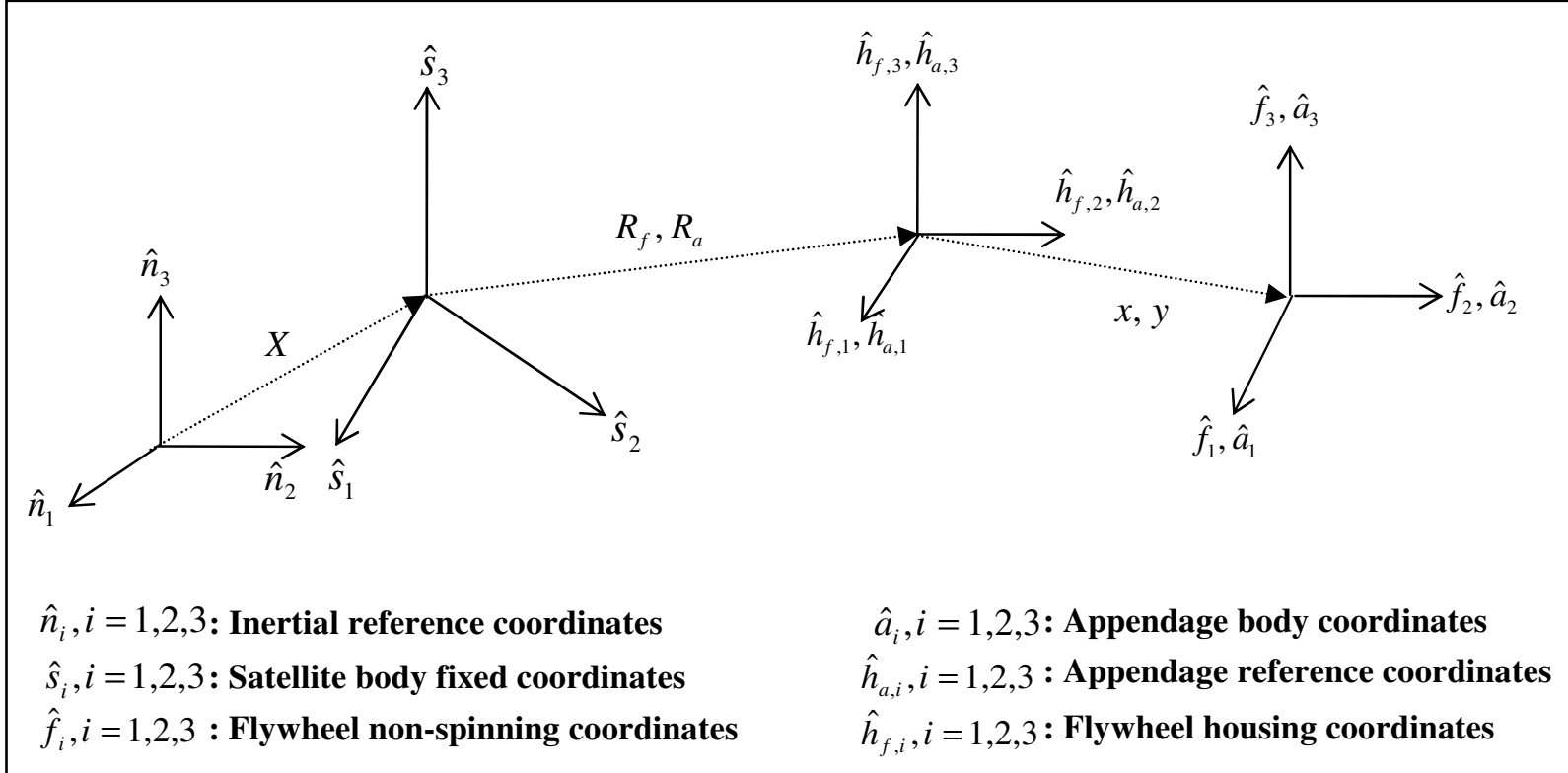

Fig. 2.2 Inertial, Satellite, Housing, Flywheel and Appendage Coordinate Systems

The mass-less, elastic beam elements connect rigid disks in the flywheel shaft and appendage models. The equations of motion for these disks are identical to the rigid body equations provided in the equation (2.5) and (2.6) except for stiffness and damping effects from finite element model. For example, if one rigid flywheel and appendage are modeled as 2 rigid disks connected by a flexible beam type element, respectively. The translational equations of motion for each flywheel and satellite disk become equation (2.7) thru (2.10) which has similar form of a rigid flywheel equation of motion written in (2.6). 


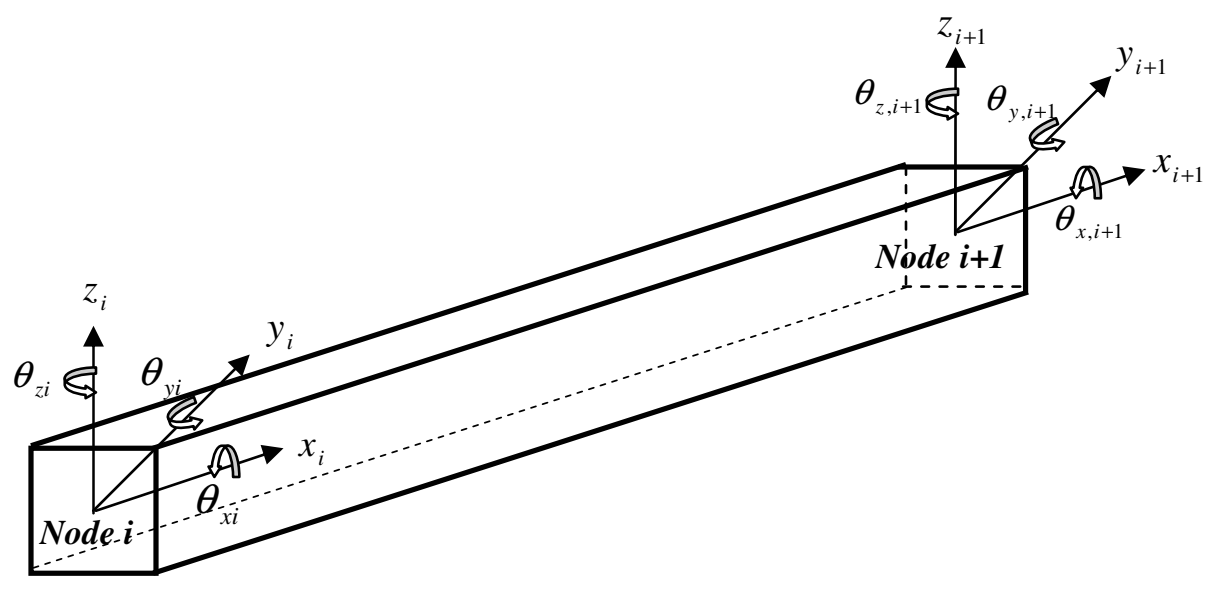

Fig. 2.3 Nodal Degrees of Freedom for a 3-D Beam Type Finite Element

The nodal rotational and translational degrees of freedom of the 2-noded, 6 degree of freedom per node beam element in Fig.2.3 are arranged in the element displacement vector with the following convention

$$
U_{e}=\left[\begin{array}{llllllllllll}
x_{i} & y_{i} & z_{i} & \theta_{x i} & \theta_{y i} & \theta_{z i} & x_{i+1} & y_{i+1} & z_{i+1} & \theta_{x, i+1} & \theta_{y, i+1} & \theta_{z, i+1}
\end{array}\right]^{T}
$$

The diagonal lumped mass matrix and stiffness matrix for the beam element are given in equation (2.2) and (2.3). It is important to note that equation (2.2) is shown only to identify the inertia associated with each DOF. The mass matrix in (2.2) is not multiplied times the $2^{\text {nd }}$ time derivative of $(2.1)$ to obtain inertia forces, which are instead obtained via the full 3D nonlinear Euler equations. Equation (2.4) describes a proportional damping matrix [30] employed to account for the damping inherent in the material.

$$
M_{e}=\operatorname{diag}\left(\left[\begin{array}{lllllllllllllll}
m_{i} & m_{i} & m_{i} & I_{p, i} & I_{t, i} & I_{t, i} & m_{i+1} & m_{i+1} & m_{i+1} & I_{p, i+1} & I_{t, i+1} & I_{t, i+1}
\end{array}\right]\right)
$$




$$
K_{e}=\left[\begin{array}{cccccccccccc}
a_{1}^{e} & 0 & 0 & 0 & 0 & 0 & -a_{1}^{e} & 0 & 0 & 0 & 0 & 0 \\
& a_{2}^{e} & 0 & 0 & 0 & a_{3}^{e} & 0 & -a_{2}^{e} & 0 & 0 & 0 & a_{3}^{e} \\
& & a_{4}^{e} & 0 & a_{5}^{e} & 0 & 0 & 0 & -a_{4}^{e} & 0 & a_{5}^{e} & 0 \\
S & & & a_{6}^{e} & 0 & 0 & 0 & 0 & 0 & -a_{6}^{e} & 0 & 0 \\
& Y & & & a_{7}^{e} & 0 & 0 & 0 & -a_{5}^{e} & 0 & a_{8}^{e} & 0 \\
& & M & & & a_{9}^{e} & 0 & -a_{3}^{e} & 0 & 0 & 0 & a_{10}^{e} \\
& & & M & & & a_{1}^{e} & 0 & 0 & 0 & 0 & 0 \\
& & & & E & & & a_{2}^{e} & 0 & 0 & 0 & -a_{3}^{e} \\
& & & & & T & & & a_{4}^{e} & 0 & -a_{5}^{e} & 0 \\
& & & & & & R & & & a_{6}^{e} & 0 & 0 \\
& & & & & & & I & & & a_{7}^{e} & 0 \\
& & & & & & & C & & & a_{9}^{e}
\end{array}\right]
$$

where

$$
\begin{array}{llll}
a_{1}^{e}=E^{e} A^{e} / L_{e} & a_{2}^{e}=12 E^{e} I_{x 3}^{e} / L_{e}^{3} & a_{3}^{e}=6 E^{e} I_{x 3}^{e} / L_{e}^{2} & a_{4}^{e}=12 E^{e} I_{x 2}^{e} / L_{e}^{3} \\
a_{5}^{e}=-6 E^{e} I_{x 2}^{e} / L_{e}^{2} & a_{6}^{e}=G^{e} J^{e} / L_{e} & a_{7}^{e}=4 E^{e} I_{x 2}^{e} / L_{e} & a_{8}^{e}=2 E^{e} I_{x 2}^{e} / L_{e} \\
a_{9}^{e}=4 E^{e} I_{x 3}^{e} / L_{e} & a_{10}^{e}=2 E^{e} I_{x 3}^{e} / L_{e} & &
\end{array}
$$

\subsubsection{Translational Equation of Motion for a Rigid Flywheel Model [29]}

From Fig. 2.2:

$$
m_{f} \ddot{X}_{f / n}^{h}=F_{f}
$$

$$
m_{f}\left\{(\ddot{x})_{h}+\left[h_{f} n\right] \ddot{X}-X_{f / s}^{h} \times\left[h_{f} s\right] \dot{\Omega}_{s}\right\}=F_{f}-2 m_{f}\left[h_{f} s\right] \Omega_{s} \times(\dot{x})_{h}-m_{f}\left\{\left[h_{f} s\right] \Omega_{s} \times\left(\left[h_{f} s\right] \Omega_{s} \times X_{f / s}^{h}\right)\right\}
$$

\subsubsection{Translational Equations of Motion for a Pair of Neighboring Rigid Disks in the Flexible Flywheel and Appendage Models}

- $i$ th Disk Translational Motion (Flywheel)

$$
\begin{aligned}
& m_{i, f}\left\{\left(\ddot{x}_{i}\right)_{h}+\left[h_{f} n\right]_{i} \ddot{X}_{i}-X_{i f / s}^{h} \times\left[h_{f} s\right]_{i} \dot{\Omega}_{i, s}\right\}+F_{i, c}+F_{i, s}= \\
& F_{i, f}-2 m_{i, f}\left[h_{f} s\right]_{i} \Omega_{i, s} \times\left(\dot{x}_{i}\right)_{h}-m_{i, f}\left\{\left[h_{f} s\right]_{i} \Omega_{i, s} \times\left(\left[h_{f} s\right]_{i} \Omega_{i, s} \times X_{i f / s}^{h}\right)\right\}
\end{aligned}
$$


- $i+1$ th Disk Translational Motion (Flywheel)

$$
\begin{aligned}
& m_{i+1, f}\left\{\left(\ddot{x}_{i+1}\right)_{h}+\left[h_{f} n\right]_{i+1} X_{i+1}-X_{i+1 f f / s}^{h} \times\left[h_{f} s\right]_{i+1} \dot{\Omega}_{i+1, s}\right\}+F_{i+1, c}+F_{i+1, s}= \\
& F_{i+1, f}-2 m_{i+1, f}\left[h_{f} s\right]_{i+1} \Omega_{i+1, s} \times\left(\dot{x}_{i+1}\right)_{h}-m_{i+1, f}\left\{\left[h_{f} s\right]_{i+1} \Omega_{i+1, s} \times\left(\left[h_{f} s\right]_{i+1} \Omega_{i+1, s} \times X_{i+1, f / s}^{h}\right)\right\}
\end{aligned}
$$

- $i$ th Disk Translational Motion (Appendage)

$$
\begin{aligned}
& m_{i, a}\left\{\left(\ddot{y}_{i}\right)_{h}+\left[h_{a} n\right]_{i} \ddot{X}_{i}-X_{i a / s}^{h} \times\left[h_{a} s\right]_{i} \dot{\Omega}_{i, s}\right\}+F_{i, c}+F_{i, s}= \\
& F_{i, a}-2 m_{i, a}\left[h_{a} s\right]_{i} \Omega_{i, s} \times\left(\dot{y}_{i}\right)_{h}-m_{i, a}\left\{\left[h_{a} s\right]_{i} \Omega_{i, s} \times\left(\left[h_{a} s\right]_{i} \Omega_{i, s} \times X_{i a / s}^{h}\right)\right\}
\end{aligned}
$$

- $i+1$ th Disk Translational Motion (Appendage)

$$
\begin{aligned}
& m_{i+1, a}\left\{\left(\ddot{y}_{i+1}\right)_{h}+\left[h_{a} n\right]_{i+1} X_{i+1}-X_{i+1 a / s}^{h} \times\left[h_{a} s\right]_{i+1} \dot{\Omega}_{i+1, s}\right\}+F_{i+1, c}+F_{i+1, s}= \\
& F_{i+1, a}-2 m_{i+1, a}\left[h_{a} s\right]_{i+1} \Omega_{i+1, s} \times\left(\dot{y}_{i+1}\right)_{h}-m_{i+1, a}\left\{\left[h_{a} s\right]_{i+1} \Omega_{i+1, s} \times\left(\left[h_{a} s\right]_{i+1} \Omega_{i+1, s} \times X_{i+1, a / s}^{h}\right)\right\}
\end{aligned}
$$

where

$$
\begin{aligned}
& x=x_{1} \hat{h}_{f, 1}+x_{2} \hat{h}_{f, 2}+x_{3} \hat{h}_{f, 3}, \quad y=y_{1} \hat{h}_{a, 1}+y_{2} \hat{h}_{a, 2}+y_{3} \hat{h}_{a, 3}, \quad R_{f}=R_{f, 1} \hat{s}_{1}+R_{f, 2} \hat{s}_{2}+R_{f, 3} \hat{s}_{3} \\
& R_{a}=R_{a, 1} \hat{s}_{1}+R_{a, 2} \hat{s}_{2}+R_{a, 3} \hat{s}_{3}, \quad X=X_{1} \hat{n}_{1}+X_{2} \hat{n}_{2}+X_{3} \hat{n}_{3}, \quad X_{f / s}^{h}=x+\left[h_{f} s\right] R_{f} \\
& \dot{X}_{f / s}^{h}=(\dot{x})_{h}+\left[h_{f} s\right] \Omega_{s} \times x+\left[h_{f} s\right]\left(\Omega_{s} \times R_{f}\right), \quad \dot{X}_{f / n}^{h}=(\dot{x})_{h}+\left[h_{f} s\right] \Omega_{s} \times x+\left[h_{f} s\right]\left(\Omega_{s} \times R_{f}\right)+\left[h_{f} n\right] \dot{X} \\
& F_{i, s}=K_{e,(1: 3,1: 12)} U_{e}, \quad F_{i+1, s}=K_{e,(7: 9,1: 12)} U_{e} \\
& T_{i, s}=K_{e,(4: 6,1,122)} U_{e}, \quad T_{i+1, s}=K_{e,(10: 12,1: 12)} U_{e} \\
& F_{i, c}=C_{e,(13,1,12)} \dot{U}_{e}, \quad F_{i+1, c}=C_{e,(79,1: 12)} \dot{U}_{e} \\
& T_{i, c}=C_{e,(46,6,112)} \dot{U}_{e}, \quad T_{i+1, c}=C_{e,(10: 12,1: 12)} \dot{U}_{e}
\end{aligned}
$$

\subsection{Rotational Motion of Flexible Flywheel and Appendage Models}

The rotational equations of motion for a rigid flywheel model are derived in a nonspinning coordinate system $[2,29]$. The flywheel angular momentum vector is given by (2.11) and the flywheel rotational equation of motion is obtained through differentiation of the angular momentum vector. 


\subsubsection{Rotational Equation of Motion for a Rigid Flywheel Model}

$$
\begin{gathered}
H_{f}=I_{f} \omega_{f} \text {, where } \omega_{f}=\Omega_{f}+[f s] \Omega_{s} \\
\dot{H}_{f}=\frac{d}{d t}\left(H_{f}\right)_{f}+\omega_{f / n} \times H_{f}=I_{f} \frac{d}{d t}\left(\Omega_{f}+[f s] \Omega_{s}\right)_{f}+\omega_{f / n} \times H_{f}=T_{f} \\
\left.I_{f} \dot{\Omega}_{f}+I_{f}[f s] \dot{\Omega}_{s}=T_{f}-\widetilde{\omega}_{f} I_{f} \Omega_{f}+\left(I_{f} \widetilde{\Omega}_{f}-\widetilde{\omega}_{f} I_{f}\right) f f s\right] \Omega_{s}
\end{gathered}
$$

\subsubsection{Rotational Equations of Motion for a Pair of Neighboring Rigid Disks in the} Flexible Flywheel and Appendage Models

- $i$ th Disk Rotational Motion (Flywheel)

$$
I_{i, f} \dot{\Omega}_{i, f}+I_{i, f}[f s]_{i} \dot{\Omega}_{i, s}+T_{i, c}+T_{i, s}=T_{i, f}-\tilde{\omega}_{i, f} I_{i, f} \Omega_{i, f}+\left(I_{i, f} \tilde{\Omega}_{i, f}-\tilde{\omega}_{i, f} I_{i, f}\right)[f s]_{i} \Omega_{i, s}
$$

- $i+1$ th Disk Rotational Motion (Flywheel)

$$
\begin{aligned}
& I_{i+1, f} \dot{\Omega}_{i+1, f}+I_{i+1, f}[f s]_{i+1} \dot{\Omega}_{i+1, s}+T_{i+1, c}+T_{i+1, s}= \\
& T_{i+1, f}-\tilde{\omega}_{i+1, f} I_{i+1, f} \Omega_{i+1, f}+\left(I_{i+1, f} \tilde{\Omega}_{i+1, f}-\tilde{\omega}_{i+1, f} I_{i+1, f}\right)[f s]_{i+1} \Omega_{i+1, s}
\end{aligned}
$$

- $i$ th Disk Rotational Motion (Appendage)

$$
\left.I_{i, a} \dot{\Omega}_{i, a}+I_{i, a}[a s]_{i} \dot{\Omega}_{i, s}+T_{i, c}+T_{i, s}=T_{i, a}-\widetilde{\omega}_{i, a} I_{i, a} \Omega_{i, a}+\left(I_{i, a} \tilde{\Omega}_{i, a}-\widetilde{\omega}_{i, a} I_{i, a}\right) t a s\right]_{i} \Omega_{i, s}
$$

- $i+1$ th Disk Rotational Motion (Appendage)

$$
\begin{aligned}
& I_{i+1, a} \dot{\Omega}_{i+1, a}+I_{i+1, a}[a s]_{i+1} \dot{\Omega}_{i+1, s}+T_{i+1, c}+T_{i+1, s}= \\
& T_{i+1, a}-\widetilde{\omega}_{i+1, a} I_{i+1, a} \Omega_{i+1, a}+\left(I_{i+1, a} \widetilde{\Omega}_{i+1, a}-\widetilde{\omega}_{i+1, a} I_{i+1, a}\right)[a s]_{i+1} \Omega_{i+1, s}
\end{aligned}
$$

\subsection{Satellite Rotational and Translational Equations of Motions}

The satellite rotational and translational motions are described in (2.18) and (2.19) in the case of no external forces. These equations are basically derived from conservation of momentum theory. 


\subsubsection{Rotational Motion (No External Torques)}

$$
\begin{aligned}
I_{s} \dot{\Omega}_{s}+\tilde{\Omega}_{s} I_{s} \Omega_{s}= & -\sum\left[\sum_{i=1}^{n}\left[f_{i} s\right]^{T} T_{f, i}\right]-\sum\left[\sum_{i=1}^{n}\left\{\left(\dot{X}_{f, i / s}^{h, i}\right) \times m_{f, i}\left(\dot{X}_{f, i / n}^{h, i}\right)+\left(X_{f, i / s}^{h, i}\right) \times\left(F_{f, i}\right)\right\}\right] \\
& =-\sum\left[\sum_{j=1}^{m}\left[a_{j} s\right]^{T} T_{a, j}\right]-\sum\left[\sum_{j=1}^{m}\left\{\left(\dot{X}_{a, j / s}^{h, j}\right) \times m_{a, j}\left(\dot{X}_{a, j / n}^{h, j}\right)+\left(X_{a, j / s}^{h, j}\right) \times\left(F_{a, j}\right)\right\}\right]
\end{aligned}
$$

\subsubsection{Translational Motion (No External Forces)}

$$
M_{s} \ddot{X}=-\sum\left[\sum_{i=1}^{n}\left[h_{f, i} n\right]^{T} F_{f, i}\right]-\sum\left[\sum_{j=1}^{m}\left[h_{a, j} n\right]^{T} F_{a, j}\right]
$$




\section{CHAPTER III}

\section{SATELLITE IPAC MANEUVER AND FEEDBACK CONTROL*}

\subsection{Overview}

The total IPAC system has mainly two different feedback control loops shown in the Fig.6.1 on p.58, which are flywheel motor control and magnetic bearing position control. This chapter will be discussed about flywheel motor control to achieve attitude control and power tracking tasks and magnetic bearing control will be dealt in the next chapter. For flywheel motor control matters in order to obtain stable satellite maneuver, the Lyapunov stability approach is employed and derived as non linear state feedback equation. As for as IPAC achievement concerned, the flywheel spin speeds should be controlled in the sense of satellite attitude control and power tracking.

The satellite reference motion is designed by "bang-bang" control which is optimal control for a rigid body minimum time maneuver. The structures of the "bang-bang" control of a rest to rest maneuver through a principal angle are presented in this chapter. The details of "bang-bang" control and "bang-off-bang" control are referenced in the [11] for near minimum time and near minimum fuel maneuver.

Closed-loop error dynamics and root-locus analysis are utilized to determine proper flywheel motor control gains. The closed-loop error dynamics could be expressed in the linearized form with reasonable approximation.

\footnotetext{
*Reprinted with permission from "MIMO Active Vibration Control of Magnetically Suspended Flywheels for Satellite IPAC Service," Park, J., 2007, Journal of Dynamic Systems, Measurement and Control, Accepted, Copyright [2008] by ASME.
} 
The torque and power distribution to each flywheel could not be determined uniquely because the satellite has more than 3 flywheel modules (underdamped system). The flywheel motor torque distribution and control gains are presented in the sections 3.3.3 and 3.3.2, respectively.

\subsection{Satellite Reference Motion Design}

Euler's Principal Axis Theorem shows that a rigid body may undergo an arbitrary three dimensional re-orientation by rotating about a single "principal" axis. A nearminimum-time control law for single axis, rest to rest maneuver of a rigid body has the form [31].

$$
f\left(\Delta t, t_{f}, t\right)=\left\{\begin{array}{c}
I \ddot{\theta}=u= \pm u_{\max } f\left(\Delta t, t_{f}, t\right) \\
\left(\frac{t}{\Delta t}\right)^{2}\left[3-2\left(\frac{t}{\Delta t}\right)\right], \quad \text { for } \quad 0 \leq t \leq \Delta t \\
1, \quad \text { for } \quad \Delta t \leq t \leq \frac{t_{f}}{2}-\Delta t \equiv t_{1} \\
1-2\left(\frac{t-t_{1}}{2 \Delta t}\right)^{2}\left[3-2\left(\frac{t-t_{1}}{2 \Delta t}\right)\right], \quad \text { for } \quad t_{1} \leq t \leq \frac{t_{f}}{2}+\Delta t \equiv t_{2} \\
-1, \quad f o r \quad t_{2} \leq t \leq t_{f}-\Delta t \equiv t_{3} \\
-1+\left(\frac{t-t_{3}}{\Delta t}\right)^{2}\left[\begin{array}{ll}
\left.3-2\left(\frac{t-t_{3}}{\Delta t}\right)\right], & \text { for } \quad t_{3} \leq t \leq t_{f}
\end{array}\right\}
\end{array}\right.
$$

where $u_{\max }$ and $\ddot{\theta}$ are one-dimensional quantities measured along the principal axis of rotation.

Integration of (3.1) yields

$$
\dot{\theta}(t)=\dot{\theta}_{0}+\frac{u_{\max }}{I} \int_{t_{0}}^{t} f\left(\Delta t, t_{f}, \tau\right) d \tau
$$




$$
\theta(t)=\theta_{0}+\left(t-t_{0}\right) \dot{\theta}_{0}+\frac{u_{\max }}{I} \int_{t_{0}}^{t} \int_{t_{0}}^{\tau_{1}} f\left(\Delta t, t_{f}, \tau_{2}\right) d \tau_{2} d \tau_{1}
$$

For rest-to-rest maneuver, we impose the boundary conditions

$$
\begin{aligned}
& \text { At time } t_{0}=0: \quad \theta(0)=\theta_{0}=0, \quad \dot{\theta}(0)=\dot{\theta}_{0}=0 \\
& \text { At time } t_{f}: \quad \theta\left(t_{f}\right)=\theta_{f}, \quad \dot{\theta}\left(t_{f}\right)=\dot{\theta}_{f}=0
\end{aligned}
$$

and upon carrying out the integrations implies in equation (3.3) and (3.4). We obtain the useful relationship.

$$
\theta_{f}=\frac{u_{\max }}{I}\left[\frac{1}{4}-\frac{1}{2} \alpha+\frac{1}{10} \alpha^{2}\right] t_{f}^{2}
$$

Let $\tilde{A}=\frac{u_{\max }}{I}$ in the equation (3.3) and (3.4). Plug (3.7) into $\tilde{A}$, then we obtain followings

$$
\tilde{A}=\frac{4 \theta_{f}}{\left(1-2 \alpha+0.4 \alpha^{2}\right) t_{f}^{2}}
$$

The above equations (3.1), (3.3) and (3.4) can be expressed by (3.9) after plugged in;

$$
\begin{gathered}
\ddot{\theta}=\tilde{A} f\left(\Delta t, t_{f}, t\right) \\
\dot{\theta}(t)=\tilde{A} \int_{t_{0}}^{t} f\left(\Delta t, t_{f}, \tau\right) d \tau \\
\theta(t)=\tilde{A} \int_{t_{0}}^{t} \int_{t_{0}}^{\tau_{1}} f\left(\Delta t, t_{f}, \tau_{2}\right) d \tau_{2} d \tau_{1}
\end{gathered}
$$

If Euler's principle axis of rotation is determined as $l$, then the corresponding angular velocity, angular acceleration and Modified Rodrigues Parameters (MRP) are given by 


$$
\Omega_{s r}(t)=l \dot{\theta}(t), \quad \dot{\Omega}_{\mathrm{sr}}(t)=l \ddot{\theta}(t) \text { and } \sigma_{s r}(t)=l \tan \left(\frac{\theta(t)}{4}\right)
$$

The satellite reference motions such as angular acceleration, angular velocity, and

Modified Rodrigues Parameter $\left(\dot{\Omega}_{s r}, \Omega_{s r}, \sigma_{s r}\right)$ can be obtained from (3.1) thru (3.10).

\subsection{Flywheel Speed Control for IPAC}

System control includes both position control for each of the magnetically supported flywheels and control of the flywheel speeds for actuation and power transfer in IPAC service. This section contains the analysis for the IPAC control law which consists of a nonlinear, state feedback, asymptotic stable [16], tracking control law derived with a Lyapunov approach [10]. The primarily purpose of feedback control is to eliminate any non-zero attitude error so the reality model tracks the designed reference motion presented in the previous chapter. The satellite angular velocity, $\Omega_{s}$, is defined in the satellite body fixed frame and satellite designed angular velocity, $\Omega_{s r}$, is coordinatized in the reference frame.

\subsubsection{IPAC Control Law}

Consider the following candidate Lyapunov function [32,33] expressed in terms of the tracking error and its time derivative in the (3.11) and its time derivative can be obtained as (3.12) from remarkable results in (3.13)

$$
\begin{gathered}
V=\frac{1}{2} \delta \omega^{T} I_{s} \delta \omega+2 k_{2} \ln \left(1+\delta \sigma^{T} \delta \sigma\right) \\
\dot{V}=\delta \omega^{T} I_{s} \delta \dot{\omega}+4 k_{2} \frac{\delta \sigma^{T} \delta \dot{\sigma}}{1+\delta \sigma^{T} \delta \sigma}=\delta \omega^{T} I_{s} \delta \dot{\omega}+4 k_{2} \frac{\delta \sigma^{T} f(\delta \sigma) \delta \omega}{1+\delta \sigma^{T} \delta \sigma}=\delta \omega^{T}\left(I_{s} \delta \dot{\omega}+k_{2} \delta \sigma\right)
\end{gathered}
$$


where $\delta \dot{\sigma}=f(\delta \sigma) \delta \omega, \quad \delta \sigma^{T} f(\delta \sigma) \delta \omega=\frac{1+\delta \sigma^{T} \delta \sigma}{4} \delta \sigma^{T} \delta \omega$

Let the term in the parenthesis of (3.12) be equal to $-k_{1} \delta \omega$, then equation (3.12) yields;

$$
\dot{V}=-k_{1} \delta \omega^{T} \delta \omega \leq 0, \text { for all } \delta \omega \text { and } k_{1}>0 \text { where } I_{s} \delta \dot{\omega}+k_{2} \delta \sigma=-k_{1} \delta \omega
$$

where $k_{1}$ is satellite angular velocity feedback gain and $k_{2}$ is a scalar gain for the attitude error feedback. The angular velocity error and its time derivative can be written as (3.15) and (3.16) in the satellite coordinates.

$$
\begin{gathered}
\delta \omega=\Omega_{s}-[s r] \Omega_{s r} \\
\delta \dot{\omega}=\dot{\Omega}_{s}-\left(\left(\frac{d}{d t}[s r]\right) \Omega_{s r}+[s r] \dot{\Omega}_{s r}\right)=\dot{\Omega}_{s}-\left(-\widetilde{\Omega}_{s}[s r] \Omega_{s r}+[s r] \dot{\Omega}_{s r}\right) \\
=\dot{\Omega}_{s}-[s r] \dot{\Omega}_{s r}+\left(\Omega_{s}\right) \times\left(\Omega_{s}-\delta \omega\right)=\dot{\Omega}_{s}-[s r] \dot{\Omega}_{s r}+\delta \widetilde{\omega} \Omega_{s} \\
\text { where } \frac{d}{d t}([s r])=\frac{d}{d t}\left([s n][r n]^{T}\right)=\frac{d}{d t}([s n])[r n]^{T}+[s n] \frac{d}{d t}\left([r n]^{T}\right)=-\tilde{\Omega}_{s}[s r]+[s r] \tilde{\Omega}_{s r}
\end{gathered}
$$

The effective torque required for the actual motion is

$$
\Gamma_{s}=I_{s} \dot{\Omega}_{s}+\tilde{\Omega}_{s} I_{s} \Omega_{s} \text { or } \dot{\Omega}_{s}=I_{s}^{-1}\left(\Gamma_{s}-\tilde{\Omega}_{s} I_{s} \Omega_{s}\right)
$$

Pre-multiply equation (3.16) by $I_{s}$, to obtain equation (3.18) (error motion). Equation (3.19) is obtained by substituting (3.17) into (3.18)

$$
\begin{gathered}
I_{s} \delta \dot{\omega}=I_{s} \dot{\Omega}_{s}-I_{s}[s r] \dot{\Omega}_{s r}+I_{s} \delta \tilde{\omega} \Omega_{s} \\
I_{s} \delta \dot{\omega}=\Gamma_{s}-\widetilde{\Omega}_{s} I_{s} \Omega_{s}-I_{s}[s r] \dot{\Omega}_{s r}+I_{s} \delta \tilde{\omega} \Omega_{s}
\end{gathered}
$$

Equating equation (3.14) and (3.19) to obtain 


$$
\begin{gathered}
I_{s} \delta \dot{\omega}=\Gamma_{s}-\tilde{\Omega}_{s} I_{s} \Omega_{s}-I_{s}[s r] \dot{\Omega}_{s r}+I_{s} \delta \tilde{\omega} \Omega_{s}=-k_{1} \delta \omega-k_{2} \delta \sigma \\
\Gamma_{s}=\tau_{s}-\sum\left[\left[f_{s}\right]^{T} T_{m t}\right]
\end{gathered}
$$

Then the flywheel control motor torques are obtained by substituting (3.21) into (3.20)

$$
\sum\left[[f s]^{T} T_{m t}\right]=\tau_{s}-\tilde{\Omega}_{s} I_{s} \Omega_{s}-I_{s}[s r] \dot{\Omega}_{s r}+I_{s} \delta \tilde{\omega} \Omega_{s}+k_{1} \delta \omega+k_{2} \delta \sigma
$$

where

$$
\begin{aligned}
\tau_{s}= & -\sum\left[[f s]^{T} T_{m b}\right]-\sum\left[\sum_{i=1}^{n}\left[h_{f, i} s\right]^{T}\left(\dot{X}_{f, i / s}^{h} \times m_{f, i} \dot{X}_{f, i / n}^{h}+X_{f, i / s}^{h} \times F_{f, i}\right)\right]-\sum\left[\sum_{j=1}^{m}\left[a_{j} s\right]^{T} T_{a, j}\right] \\
& -\sum\left[\sum_{j=1}^{m}\left[h_{a, j} s\right]^{T}\left(\dot{X}_{a, j / s}^{h} \times m_{a, j} \dot{X}_{a, j / n}^{h}+X_{a, j / s}^{h} \times F_{a, j}\right)\right]
\end{aligned}
$$

Equation (3.22) indicates that required flywheel motor torque to track designed reference motion. The way selecting satellite attitude and angular velocity error feedback gains, $k_{1}$ and $k_{2}$, will be discussed in the next chapter. The Lyapunov function $V$ is positive definite and radially unbounded in terms of the tracking errors. The time derivative of $V$ given by (3.14) is negative definite without external torques. Therefore the departure motion (3.19) and kinematical equation for the departure motion (3.13) with the feedback motor torque control law (3.22) are also asymptotically stable in the absence of external torques. In the presence of a disturbing external torque, the satellite body angular velocity errors still decay to zero. However, the attitude error will converge to a finite offset depending upon attitude error control gain $\left(k_{2}\right)$. It can be also reduced by choosing a large attitude feedback gain [11]. 


\subsubsection{Flywheel Motor Control Gain Selection [11]}

The flywheel motor control gains, $k_{1}$ (satellite angular velocity control gain) and $k_{2}$ (satellite attitude control gain) can be obtained from closed-loop error dynamics and root-locos analysis. Assuming no external torque case, the closed-loop dynamics can be written as differential form in the equation (3.14).It can be recognized that this equation depends on angular velocity and attitude error. If satellite attitude error, $\delta \sigma$, is zero, then the poles of equation (3.14) could be selected arbitrary by $k_{1}$. The differential equation for $\delta \sigma$ depends quadratically on $\delta \sigma$ which is given the first equation of (3.13). However, this quadratic equation can be approximated by linearizing about $\delta \sigma=0$ as shown equation (3.23)

$$
\delta \dot{\sigma} \approx \frac{\delta \omega}{4}
$$

After combining equation (3.14) and (3.23), the following closed-loop error dynamic equation can be obtained.

$$
\left\{\begin{array}{c}
\delta \dot{\sigma} \\
\delta \dot{\omega}
\end{array}\right\}=\left[\begin{array}{cc}
0_{3 \times 3} & 0.25 I_{3 \times 3} \\
-k_{2} I_{s}^{-1} & -k_{1} I_{s}^{-1}
\end{array}\right]\left\{\begin{array}{c}
\delta \sigma \\
\delta \omega
\end{array}\right\}
$$

The root-locos method could be utilized to obtain the poles of equation (3.24) given inertia matrix. If the inertia matrix and the angular velocity control gain matrix $k_{1}$ are selected to be diagonal matrice, the equation (3.24) can be decoupled into 6 separate equations as equation (3.25).

$$
\left\{\begin{array}{c}
\delta \dot{\sigma}_{i} \\
\delta \dot{\omega}_{i}
\end{array}\right\}=\left[\begin{array}{cc}
0 & 1 / 4 \\
-k_{2}\left(I_{s}^{i}\right)^{-1} & -k_{1}^{i}\left(I_{s}^{i}\right)^{-1}
\end{array}\right]\left\{\begin{array}{c}
\delta \sigma_{i} \\
\delta \omega_{i}
\end{array}\right\} \text { where } i=1,2,3
$$


Also the roots of equation (3.25) can be solved as

$$
\lambda_{1,2}=-\frac{1}{2}\left[\frac{k_{1}}{I_{s}^{i}} \pm \sqrt{-\frac{k_{2}}{I_{s}^{i}}+\left(\frac{k_{1}^{i}}{I_{s}^{i}}\right)^{2}}\right]
$$

Fig. 3.1 plots the root - locos of equation (3.26). The attitude feedback control gain, $k_{2}$, can be selected only one (because of scalar), however, the angular velocity error feedback control gain, $k_{1}$, can be chosen for each body axis. If the closed-loop dynamics is slightly underdamped system, the angular velocity gains can be expressed in terms of the controller decay time constants, $T_{c} .[30]$ and the scalar attitude feedback control gain, $k_{2}$, has the condition for the closed-loop underdamped system. The following equations (3.27) and (3.28) indicate the expression of $T_{c}$ and condition of $k_{2}$, respectively.

$$
\begin{array}{ll}
k_{1}^{i}=2 I_{s}^{i} \frac{\ln 2}{T_{c}}, & i=1,2,3 \\
k_{2}>\frac{\left(k_{1}^{i}\right)^{2}}{I_{s}^{i}}, & i=1,2,3
\end{array}
$$

It can be recognizable that $k_{1}^{i}$ and $k_{2}$ determine whether the closed -loop system is over, critically or underdamped. Once the system is selected as underdamped, then only $k_{1}^{i}$ determines how fast a state error will decay. The simulation results in the Chapter VI, the controller decay time, $T_{c}$, is chosen as 4 [sec]. The linearized equation of (3.23) and the assumption of a diagonal satellite inertia matrix are two approximation of this analysis. Since the linearization of the Modified Rodrigues Parameters is valid for four 
times the rotational range of the Euler angles and the off-diagonal terms in the inertia matrix are usually very small compared to the diagonal terms [11].

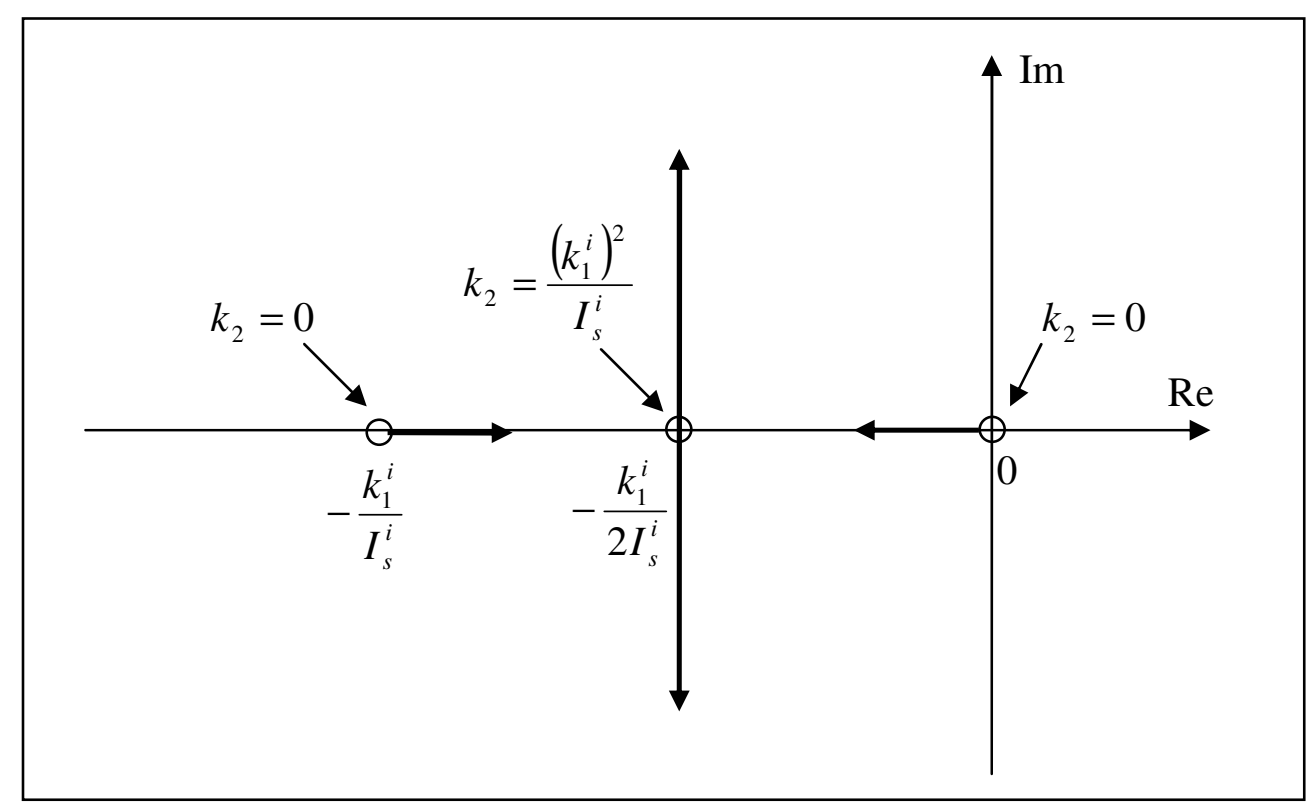

Fig. 3.1 Root -Locus Plot of the Decoupled, Linearized Error Dynamics

\subsubsection{Torque Distributions and Power Tracking [1]}

The individual flywheel motor torques and the torque required by the satellite for attitude control are related by;

$$
T_{s}=A T_{m t}
$$

where $T_{m t}$ and $T_{s}$ are the required motor torques applied to the flywheel and satellite, respectively and $A$ is the $3 \times n$ system configuration matrix ( $n$ is number of flywheel) with columns equal to the unit vectors of the flywheel housing coordinate axes. The solution of (3.23), $T_{m t}$, is a linear combination of vectors belongs to the range space of 
matrix $A$. If the number of flywheel modules is less than 3 , the system is overdetermined and a solution may not exist. If the number is 3 , the solution is uniquely determined and if the number of flywheel modules exceeds 3 , the system is underdetermined and there exist an infinite number of solutions. For this paper, the satellite has 4 flywheel modules which form an underdetermined system. One useful solution is the minimum norm solution obtained by using a pseudo-inverse. The general solution for $T_{m t}$ is given by

$$
T_{m t}=A^{+} T_{s}+T_{n} \text { where } A^{+}=A^{T}\left(A A^{T}\right)^{-1}
$$

The vector $A^{+} T_{s}$ belongs to the range space of $A^{T}$ and $T_{n}$ belongs to the null space of $A$, in other words, $A T_{n}=0$ so $T_{n}$ does not affect the satellite motion. Simultaneous attitude control and power tracking require that the torque satisfy the following set of linear equations.

$$
\left(\begin{array}{c}
A \\
\omega_{f}^{T}
\end{array}\right) T_{m t}=\left(\begin{array}{c}
T_{s} \\
P
\end{array}\right)
$$

The second constraint of (3.25) is written as $\omega_{f}^{T}\left(A^{+} T_{s}+T_{n}\right)=P$

Define the modified power as

$$
P_{m}=P-\omega_{f}^{T} A^{+} T_{s}=\omega_{f}^{T} T_{n}
$$

The power torque, $T_{n}$, belongs to the null space of the configuration matrix $A$; therefore there exists a vector $\eta$, in the null space which satisfies, 


$$
T_{n}=P_{n} \eta
$$

where $P_{n}=I_{n \times n}-A^{T}\left(A A^{T}\right)^{-1} A$ is the orthogonal projection matrix onto the null space of $A$. Thus equation (3.26) can be expressed by $\omega_{f}^{T} P_{n} \eta=P_{m}$ which after substituting (3.27), has the minimum norm solution

$$
\eta=P_{n} \omega_{f}\left(\omega_{f}^{T} P_{n} \omega_{f}\right)^{-1} P_{m}
$$

Finally, the power tracking torque is given by equation (3.29)

$$
T_{n}=P_{n} \omega_{f}\left(\omega_{f}^{T} P_{n} \omega_{f}\right)^{-1} P_{m}
$$




\section{CHAPTER IV}

\section{MAGNETIC BEARING SUSPENSION SYSTEM WITH MIMO (GYRO) CONTROL*}

\subsection{Overview}

The high speed flywheels will be suspended by magnetic bearings (MB) which have minimal loss, do not require lubrication and operate very well in a vacuum. In previous IPAC control algorithm model assumed that the flywheels are mounted on the satellite with infinite stiffness which can execute only rotational motion. In the MB feedback control point, the flywheel needs additional degrees of freedom (This chapter presents 5 axis MB feedback control loops).

The MB control algorithm is complicated by the presence of speed dependent poles that result from gyroscopic moments of the spinning, vibrating shafts. The effect of speed dependent poles is magnified as an increased energy density demand on the flywheel is met by increasing the ratio $\left(I_{P} / I_{T}\right)$ of the polar to transverse mass moments of inertia of the spinning rotors. The rigid body gyroscopic poles asymptotically approach $0[\mathrm{~Hz}]$ (backward pole) and $\left(I_{P} / I_{T}\right)$ times spin frequency (forward pole) producing a very low frequency pole and a very high frequency pole for $I_{P} / I_{T}>1$. The forward conical pole's frequency increase with speed and need phase lead and higher derivative gain at the high frequency, furthermore, time derivative of current, $L \cdot d i / d t$,

\footnotetext{
*Reprinted with permission from "MIMO Active Vibration Control of Magnetically Suspended Flywheels for Satellite IPAC Service," Park, J., 2007, Journal of Dynamic Systems, Measurement and Control, Accepted, Copyright [2008] by ASME.
} 
gets higher in the power amplifier causing current and voltage saturation. This complicates the control task since increased active damping (derivative gain) is ineffective at low frequencies and causes noise amplification at high frequency. As the results, the general single input single output (SISO) control is not appropriate in the case of higher polar and transverse moment of inertia ratio to lower the frequency of a forward conical mode.

Effective MB control then requires a shift in strategy from providing phase lead by derivative gain changes to canceling gyroscopic torques utilizing a MIMO (Multiple Input - Multiple Output) control approach. MIMO will lower the frequency of the forward conical mode by canceling some of the gyroscopic moment which requires less gain at the high frequency and coil voltage in the power amplifier will not saturate. The details of MIMO control strategy will be discussed in the sections 4.2 to 4.7 .

In general a magnetic bearing (MB) suspension system includes position sensors, feedback controllers, filters, power amplifiers and MB actuators. Each component will be briefly presented in this chapter. Fig.4.1 and 4.2 show feedback diagram of a typical magnetic suspension system and a diagram of a flywheel with a magnetic bearing suspension, respectively. 


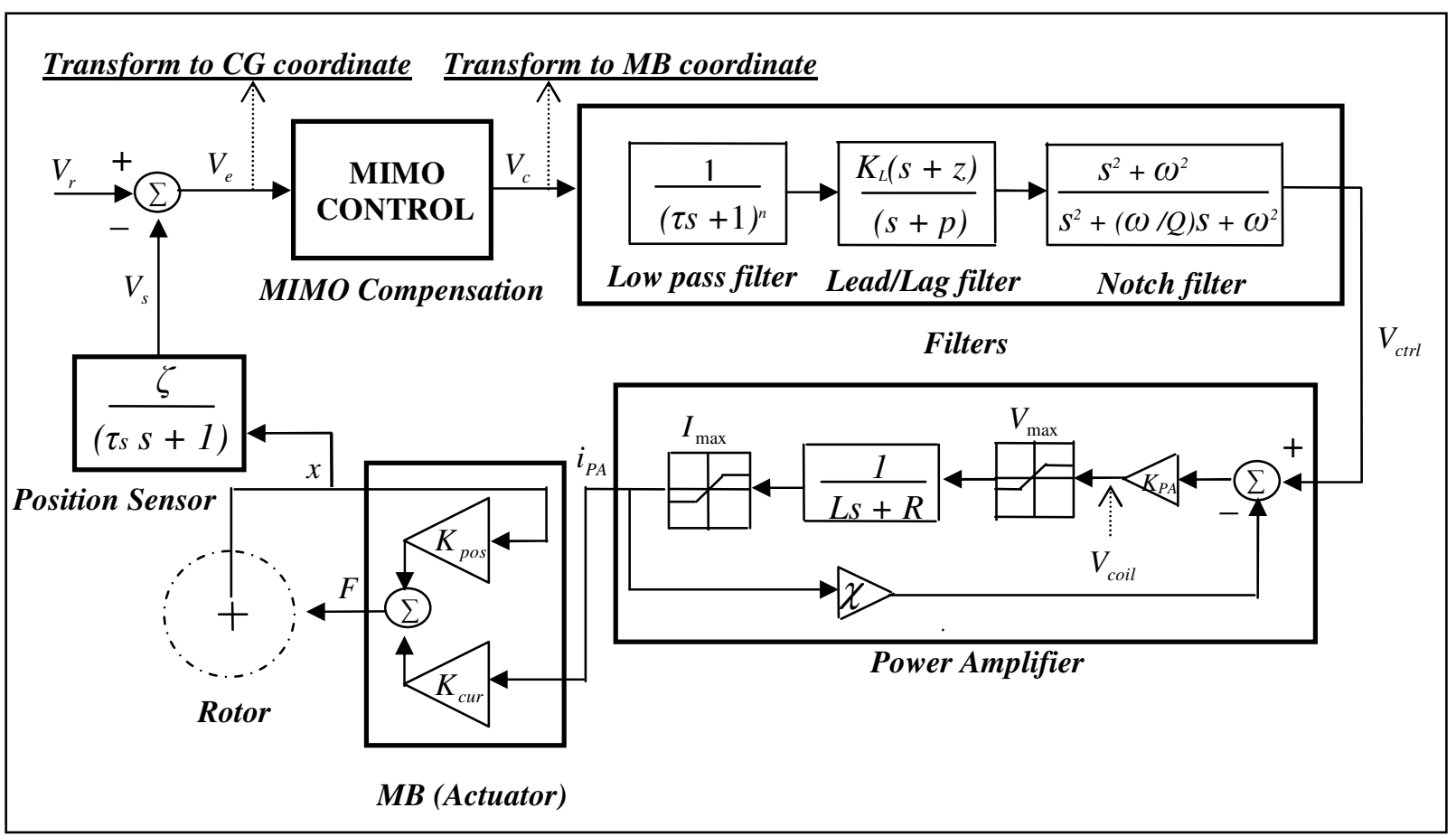

Fig. 4.1 MB Suspension System Feedback Control Diagram for MIMO (GYRO)

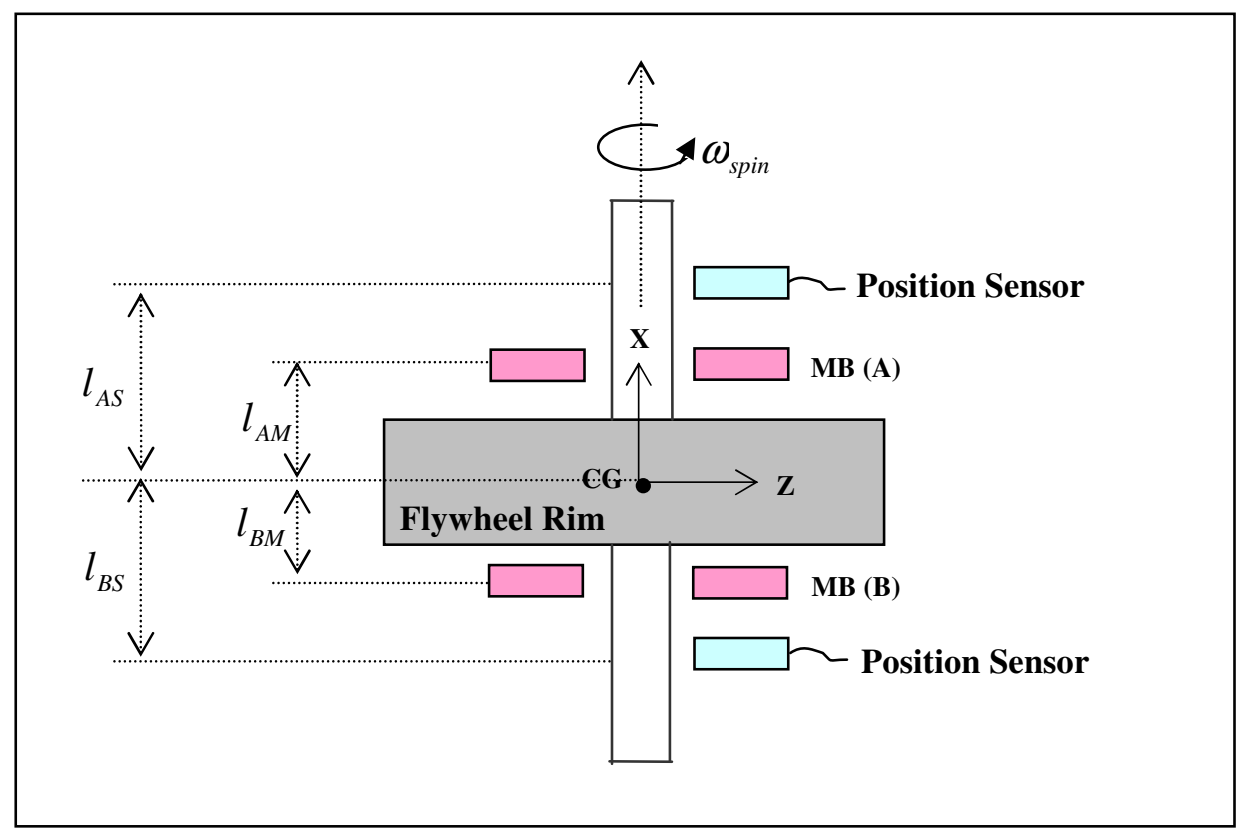

Fig. 4.2 Flywheel System with Magnetic Bearing (MB) Suspension 
The flywheel's center of gravity, "CG", coordinates include the center of gravity translational motions $\left(y\right.$ and $z$ ) and the rotational motions of shaft's rigid body $\left(\theta_{y}, \theta_{z}\right)$ shown below. The " $y-z$ " coordinates, referred to in the introduction as "MB" coordinates, are $\left(y_{A}, z_{A}, y_{B}, z_{B}\right)$ as shown in the following Fig. 4.3 and typically refer to the shaft motions at the sensor and/or actuator locations. The following analysis relates the "CG" and "MB" coordinates and equation (4.1) indicates the relationship between two different coordinate systems.

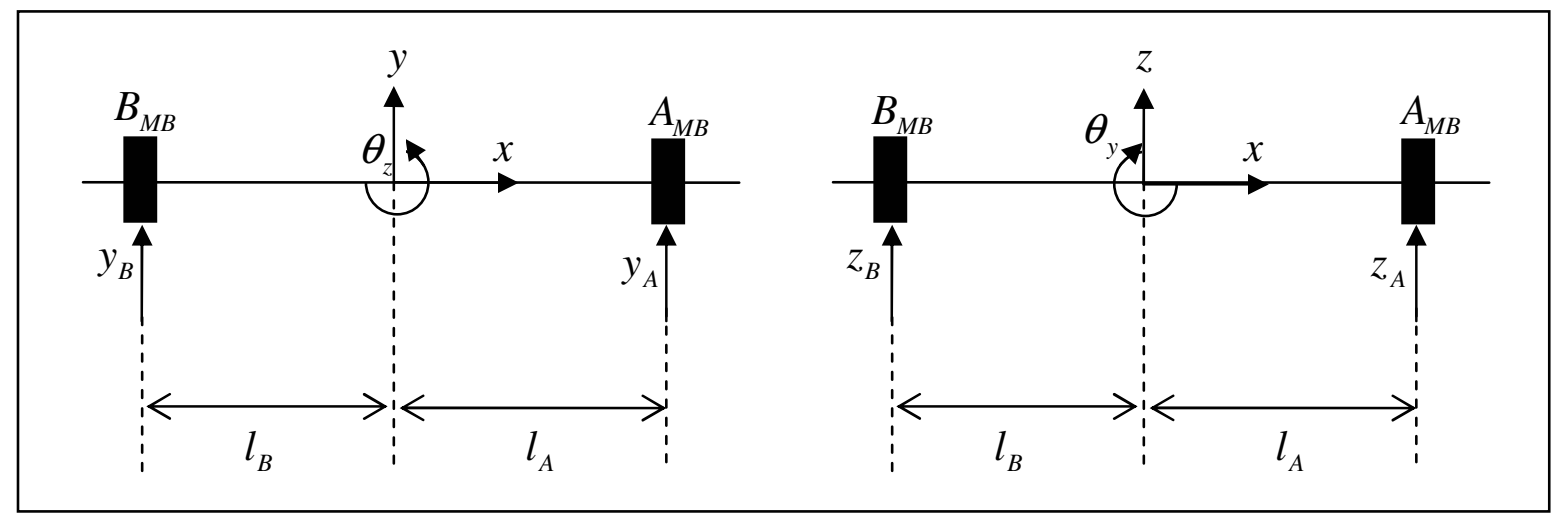

Fig. 4.3"CG" and "MB" Coordinates

$$
\left[\begin{array}{l}
y_{A} \\
z_{A} \\
y_{B} \\
z_{B}
\end{array}\right]=\left[\begin{array}{cccc}
1 & 0 & 0 & l_{A} \\
0 & -l_{A} & 1 & 0 \\
1 & 0 & 0 & -l_{B} \\
0 & l_{B} & 1 & 0
\end{array}\right]\left[\begin{array}{c}
y \\
\theta_{y} \\
z \\
\theta_{z}
\end{array}\right]
$$

where $\left[\begin{array}{llll}y_{A} & z_{A} & y_{B} & z_{B}\end{array}\right]^{T}$ and $\left[\begin{array}{llll}y & \theta_{y} & z & \theta_{z}\end{array}\right]^{T}$ are MB Coordinate and CG Coordinate, respectively. 


\subsection{Position Sensor}

Magnetic suspensions typically utilize eddy current, optical or reluctance based sensors. Approximately, the transfer function of position sensors could be expressed as a linear first order form shown in the Fig.4.1 where $\tau_{s}$ is time constant determined by cutoff frequency of the sensor characteristic and $\zeta$ is the sensor gain. The bandwidths of these devices are typically $>5[\mathrm{KHz}]$ so they are treated as ideal, infinite bandwidth devices, with sensitivity-gain.

\subsubsection{Voltage and Displacement Errors at Position Sensor}

Fig.4.4 shows the orthogonal sensor pairs at the A and B bearing position and the conversion of the position errors to voltage errors for input to the feedback controller stage.

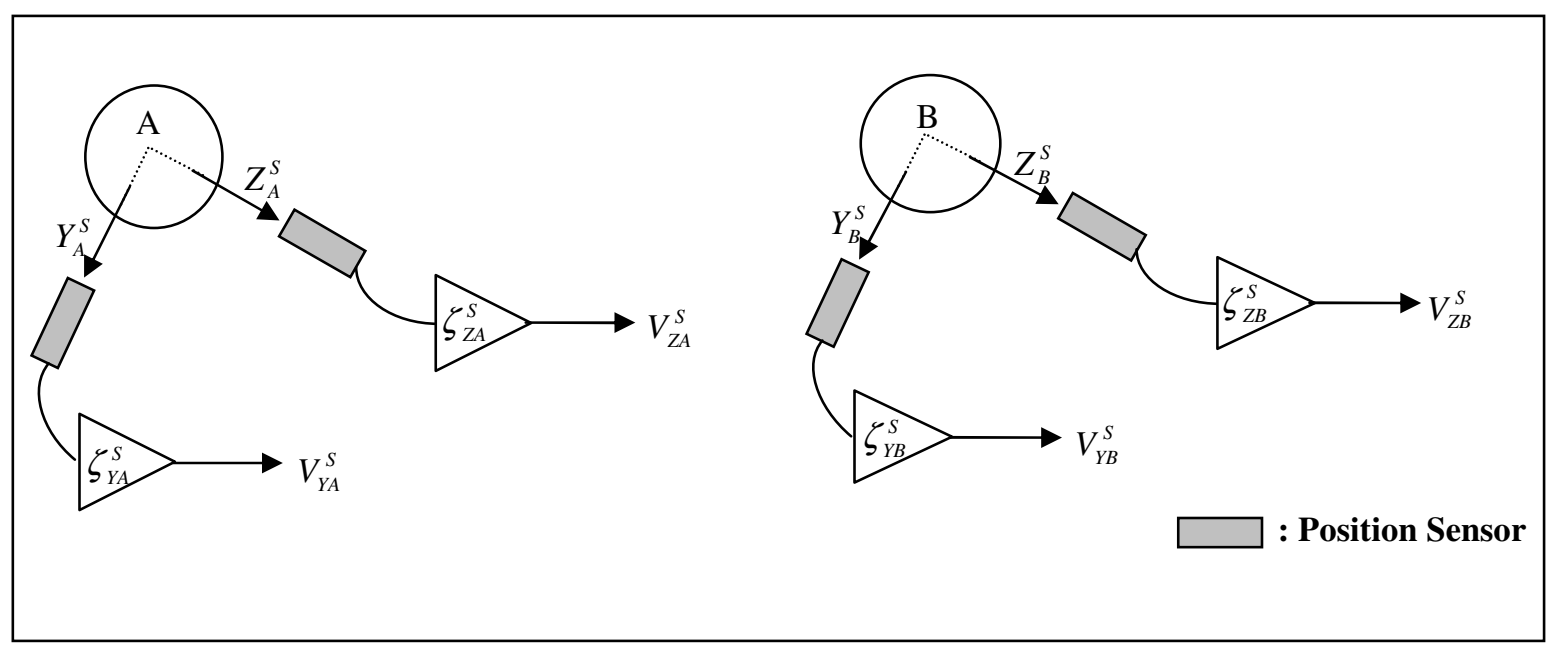

Fig. 4.4 Position Sensor Output Voltages

The voltage errors can be expressed in terms of the position errors as shown in (4.2): 


$$
\begin{gathered}
\Delta^{V}=\left\{\begin{array}{l}
\Delta_{Y A}^{V} \\
\Delta_{Y B}^{V} \\
\Delta_{Z A}^{V} \\
\Delta_{Z B}^{V}
\end{array}\right\}=\left\{\begin{array}{c}
V_{Y A}^{S}-V_{Y A}^{S, T} \\
V_{Y B}^{S}-V_{Y B}^{S S T} \\
V_{Z A}^{S}-V_{Z A}^{S, T} \\
V_{Z B}^{S}-V_{Z B}^{S, T}
\end{array}\right\}=\left\{\begin{array}{l}
\zeta_{Y A}^{S} \cdot Y_{A}^{S}-\zeta_{Y A}^{S} \cdot Y_{A}^{S, T} \\
\zeta_{Y B}^{S} \cdot Y_{B}^{S}-\zeta_{Y B}^{S} \cdot Y_{B}^{S, T} \\
\zeta_{Z A}^{S} \cdot Z_{A}^{S}-\zeta_{Z A}^{S} \cdot Z_{A}^{S, T} \\
\zeta_{Z B}^{S} \cdot Z_{B}^{S}-\zeta_{Z B}^{S} \cdot Z_{B}^{S, T}
\end{array}\right\}=\left[\begin{array}{cccc}
\zeta_{Y A}^{S} & 0 & 0 & 0 \\
0 & \zeta_{Y B}^{S} & 0 & 0 \\
0 & 0 & \zeta_{Z A}^{S} & 0 \\
0 & 0 & 0 & \zeta_{Z B}^{S}
\end{array}\right]\left\{\begin{array}{l}
e_{Y A} \\
e_{Y B} \\
e_{Z A} \\
e_{Z B}
\end{array}\right\} \\
\text { or } \Delta^{V}=\zeta^{S} \cdot \bar{e}_{S} \\
\text { where } \zeta^{S}=\left[\begin{array}{cccc}
\zeta_{Y A}^{S} & 0 & 0 & 0 \\
0 & \zeta_{Y B}^{S} & 0 & 0 \\
0 & 0 & \zeta_{Z A}^{S} & 0 \\
0 & 0 & 0 & \zeta_{Z B}^{S}
\end{array}\right], \bar{e}_{S}=\left\{\begin{array}{l}
Y_{A}^{S}-Y_{A}^{S, T} \\
Y_{B}^{S}-Y_{B}^{S, T} \\
Z_{A}^{S}-Z_{A}^{S, T} \\
Z_{B}^{S}-Z_{B}^{S, T}
\end{array}\right\}=\left\{\begin{array}{l}
e_{Y A} \\
e_{Y B} \\
e_{Z A} \\
e_{Z B}
\end{array}\right\}
\end{gathered}
$$

\subsubsection{Motion Coordinate Transformation}

Fig.4.5 presents a diagram to determine the approximate rigid body motion coordinates transformation from the measured $\mathrm{MB}$ coordinates voltage error at the position sensors. This approach can be justified since the flexible modes are typically above $1[\mathrm{KHz}]$. The rigid-rotor model frequency analysis is provided in the section 6.3 .

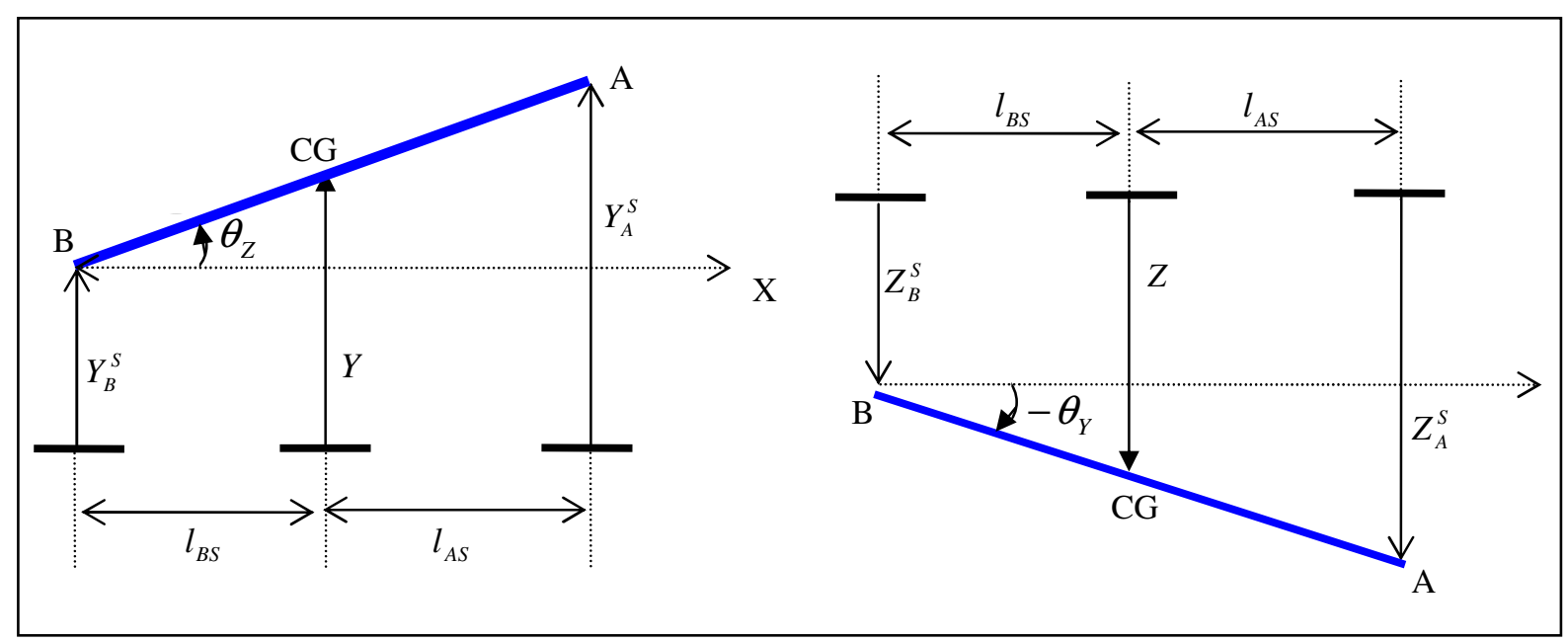

Fig.4.5 Motions Coordinate Transformation 
The approximate rigid body motion coordinates are given by (4.3):

$$
\begin{gathered}
Y=\left(\frac{l_{B S}}{l_{A S}+l_{B S}}\right) \cdot Y_{A}^{S}+\left(\frac{l_{A S}}{l_{A S}+l_{B S}}\right) \cdot Y_{B}^{S}, \quad \theta_{Z} \cong \frac{Y_{A}^{S}-Y}{l_{A S}}=\left(\frac{1}{l_{A S}}\right) \cdot Y_{A}^{S}-\left(\frac{1}{l_{A S}}\right) \cdot Y \\
Z=\left(\frac{l_{B S}}{l_{A S}+l_{B S}}\right) \cdot Z_{A}^{S}+\left(\frac{l_{A S}}{l_{A S}+l_{B S}}\right) \cdot Z_{B}^{S}, \quad \theta_{Y} \cong-\frac{Z_{A}^{S}-Z}{l_{A S}}=-\left(\frac{1}{l_{A S}}\right) \cdot Z_{A}^{S}+\left(\frac{1}{l_{A S}}\right) \cdot Z
\end{gathered}
$$

Voltage errors in the $Y, \theta_{Z}$ and $Z, \theta_{Y}$ coordinates ("CG") are expressed in terms of the sensor error voltages from (4.2) and (4.3) as

$$
\begin{array}{ll}
e_{Y}=Y-Y^{T}=\gamma_{Y A} \cdot \Delta_{Y A}^{V}+\gamma_{Y B} \cdot \Delta_{Y B}^{V}, & e_{\theta Z}=\theta_{Z}-\theta_{Z}^{T}=\psi_{\theta Z 1} \cdot \Delta_{Y A}^{V}+\psi_{\theta Z 2} \cdot e_{Y} \\
e_{Z}=Z-Z^{T}=\gamma_{Z A} \cdot \Delta_{Z A}^{V}+\gamma_{Z B} \cdot \Delta_{Z B}^{V}, & e_{\theta Y}=\theta_{Y}-\theta_{Y}^{T}=\psi_{\theta Y 1} \cdot \Delta_{Z A}^{V}+\psi_{\theta Y 2} \cdot e_{Z}
\end{array}
$$

A matrix form of these equations is given by the following

$$
\begin{gathered}
\bar{e}_{C G}=\left\{\begin{array}{c}
e_{Y} \\
e_{\theta Y} \\
e_{Z} \\
e_{\theta Z}
\end{array}\right\}=\left[\begin{array}{cccc}
\gamma_{Y A} & \gamma_{Y B} & 0 & 0 \\
0 & 0 & \psi_{\theta Y 1}+\psi_{\theta Y 2} \cdot \gamma_{Z A} & \psi_{\theta Y 2} \cdot \gamma_{Z B} \\
0 & 0 & \gamma_{Z A} & \lambda_{Z B} \\
\psi_{\theta Z 1}+\psi_{\theta Z 2} \cdot \gamma_{Y A} & \psi_{\theta Z 2} \cdot \gamma_{Y B} & 0 & 0
\end{array}\right]\left\{\begin{array}{c}
\Delta_{Y A}^{V} \\
\Delta_{Y B}^{V} \\
\Delta_{Z A}^{V} \\
\Delta_{Z B}^{V}
\end{array}\right\} \\
\text { or } \bar{e}_{C G}=T_{D C T} \cdot \Delta^{V}
\end{gathered}
$$

where $\gamma_{Y A}=\frac{l_{B S}}{\left(l_{A S}+l_{B S}\right) \zeta_{Y A}^{S}}, \quad \gamma_{Y B}=\frac{l_{A S}}{\left(l_{A S}+l_{B S}\right) \zeta_{Y B}^{S}}, \quad \psi_{\theta Z 1}=\frac{1}{l_{A S} \cdot \zeta_{Y A}^{S}}, \quad \psi_{\theta Z 2}=\frac{-1}{l_{A S}}$

$$
\gamma_{Z A}=\frac{l_{B S}}{\left(l_{A S}+l_{B S}\right) \zeta_{Z A}^{S}}, \quad \gamma_{Z B}=\frac{l_{A S}}{\left(l_{A S}+l_{B S}\right) \zeta_{Z B}^{S}}, \quad \psi_{\theta Y 1}=\frac{-1}{l_{A S} \cdot \zeta_{Z A}^{S}}, \quad \psi_{\theta Y 2}=\frac{1}{l_{A S}}
$$




\subsection{Power Amplifier}

The power amplifier transforms controller output voltages $\left(V_{c t r l}\right)$ to currents $\left(i_{P A}\right)$ that flow through the magnetic bearing coils from the Fig. 4.1. Pulse width modulated (PWM) servo amplifiers are commonly used in the MB system due to low power consumption and accurate tracking of the demanded currents. A simplified feedback model of a servo amplifier including nonlinearities such as voltage and current saturation is shown in the Fig. 4.1. The closed loop system transfer function of a servo power amplifier may be represented in a simplified form with proportional gain $\left(K_{P A}\right)$, feedback gain $(\mathcal{X})$, coil inductance and resistance $(L, R)$ as (4.6).

Fig. 4.6 describes that typical first order representation of PWM including current servo transfer function which plotted in the dashed line. The current servo dynamics could be model with coil voltage, $V_{\text {coil }}$, resistance, $R$, and inductance, $L$, as mentioned before; coil voltage will be saturated at the high frequency because inductance term in the voltage expression could be large. The high frequency of pole could not be lowered without canceling the gyroscopic moments in the high energy density flywheel case.

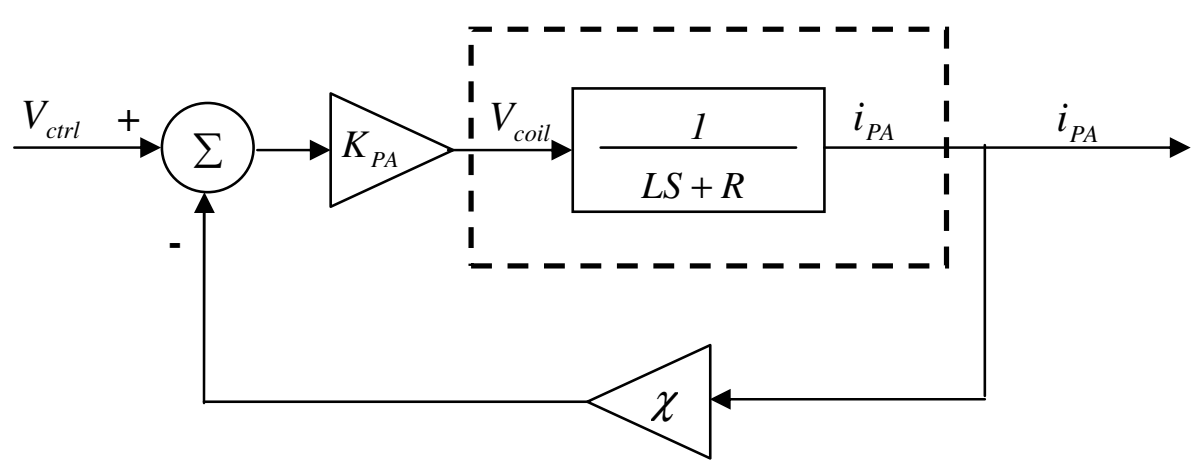

Fig. 4.6 First Order Transfer Function of PWM 


$$
G_{P A}(s)=\frac{i_{P A}}{V_{c t r l}}=\frac{K_{P A}}{L S+\left(R+\chi \cdot K_{P A}\right)}
$$

where $K_{P A}$ and $\chi$ can be selected by matching the transfer function $\mathrm{t}$ a first order filter considering the overall gain and bandwidth [2].

\subsubsection{Control Currents}

The dynamics (bandwidth) of the servo power amplifier is neglected for sake of illustration of the IPAC and AMB system vibration control. Therefore the actions of the power amplifiers may be approximated by control voltage multiply power amplifier gain which is shown (4.7) in the matrix form.

$$
\bar{i}_{P A}=\left[\begin{array}{l}
i_{Y A} \\
i_{Y B} \\
i_{Z A} \\
i_{Z B}
\end{array}\right]=\left[\begin{array}{cccc}
K_{Y A}^{P A} & 0 & 0 & 0 \\
0 & K_{Y B}^{P A} & 0 & 0 \\
0 & 0 & K_{Z A}^{P A} & 0 \\
0 & 0 & 0 & K_{Z B}^{P A}
\end{array}\right]\left\{\begin{array}{l}
V_{Y A} \\
V_{Y B} \\
V_{Z A} \\
V_{Z B}
\end{array}\right\} \quad \text { or } \quad \bar{i}_{P A}=\bar{K}^{P A} \cdot \bar{V}_{C T R L}
$$

\subsection{Magnetic Bearing - Actuator}

The forces produced by a MB actuator on the spinning flywheel shaft are nonlinear function of currents and shaft's relative position in the actuator clearance space. A MB actuator for satellite application will most likely incorporate permanent magnets to supply a bias field to minimize ohmic losses. This MB type has flux paths and other features that require a more complex model. Thus for the sake of the illustration purposes assume that an electromagnetic biased MB is utilized. Fig.4.6 [2] shows one axis of this $\mathrm{MB}$ actuator including coils, forces, and their currents. Equation (4.8) provides a representative form for this force when produced by an electromagnet biased, opposing pole, heteropolar type MB. The way to obtain the (4.8) and (4.9) will be briefly 
discussed in the following

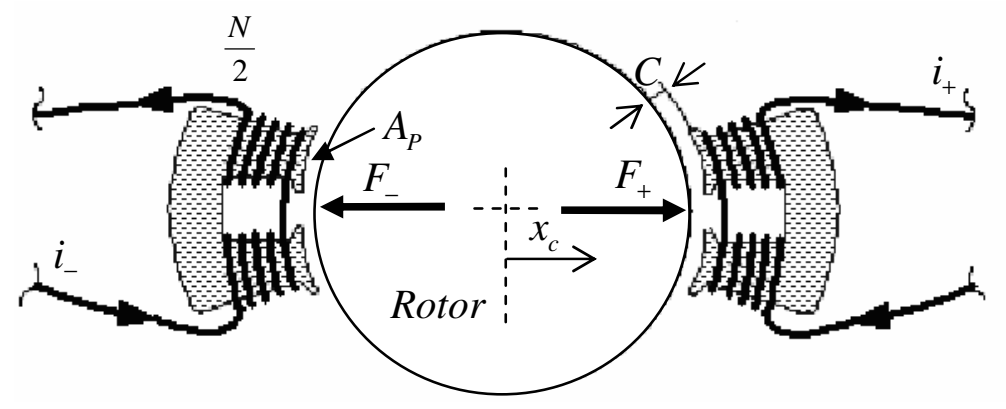

Fig. 4.7 C-core Electromagnet and Rotor Lamination Stack

The total magnetic bearing force produced in the Fig.4.7 including magnetic flux density $(B)$, cross section area $(A)$ and magnetic field constant $\left(\mu_{0}\right)$ can be written as (4.8) [34] and flex density $(B)$ can be obtained simple form by Ampere's law and conservation of flux in the circuit that is $\Phi=B_{j} A_{j}$.

Ampere's law is $\oint H d l=N \cdot i$ and can be written as $H_{s} \cdot l_{s}+H_{r} \cdot l_{r}+2 H_{o} c=N \cdot i$ from Fig. 4.8(a). The flux intensity $\left(H_{j}\right)$ can be also converted as $B_{j} / \mu_{j}$ based on linear range of $B-H$ curve. Therefore, it can be obtained the following relationship (4.9) thru (4.11) from above equation and Fig.4.8(a).

$$
\begin{gathered}
F=\frac{B_{+}^{2} A_{p}}{\mu_{0}}-\frac{B_{-}^{2} A_{p}}{\mu_{0}} \\
\frac{B_{s}}{\mu_{s}} l_{s}+\frac{B_{r}}{\mu_{r}} l_{r}+2 \frac{B_{o}}{\mu_{o}} c=N \cdot i
\end{gathered}
$$




$$
\begin{gathered}
\frac{\Phi}{\mu_{s} A_{s}} l_{s}+\frac{\Phi}{\mu_{r} A_{r}} l_{r}+2 \frac{\Phi}{\mu_{o} A_{o}} c=N \cdot i, \\
R_{s} \Phi+R_{r} \Phi+2 R_{o} \Phi=N \cdot i
\end{gathered}
$$

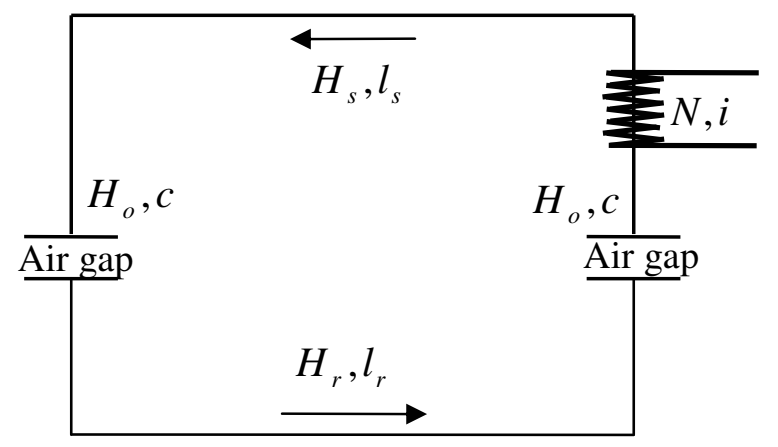

(a)

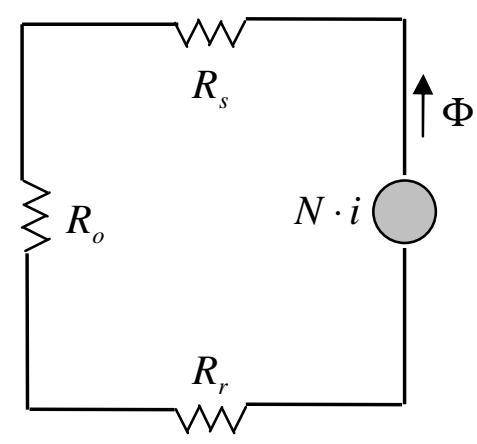

(b)

Fig. 4.8 Equivalent Magnetic Circuit

where $R_{j}=\frac{l_{j}}{\mu_{j} A_{j}}$ and for a good magnetic conducting material $\mu_{s}>\mu_{o}, \mu_{r}>\mu_{o}$, yields $R_{s}<<R_{o}$ and $R_{r} \ll<R_{o}$, then the equation (4.11) can be shorten as $2 R_{o} \Phi=N \cdot i$ from equivalent magnetic circuit shown Fig.4.8(b), therefore, the flux density $(B)$ in (4.8) can be derived from equating $\Phi=\frac{N \cdot i}{2 R_{o}}$ and $\Phi=B_{o} A_{o}$, as equation (4.12) then after inserts (4.12) into (4.8); the magnetic force can be expressed as (4.13) where $i^{+}=i_{b}+i_{c}$ and $i^{-}=i_{b}-i_{c}$.

$$
B_{o}=\frac{N_{i} \cdot i}{2 R_{o} A_{o}}
$$




$$
F=\frac{1}{4} N^{2} \mu_{0} A_{p}\left\{\frac{\left(i_{b}+i_{c}\right)^{2}}{\left(c-x_{c}\right)^{2}}-\frac{\left(i_{b}-i_{c}\right)^{2}}{\left(c+x_{c}\right)^{2}}\right\}
$$

The standard linearized form for the magnetic bearing force expression can be written as (4.14). The MB position stiffness $\left(K_{p o s}\right)$ and current stiffness $\left(K_{c u r}\right)$ are obtained by differentiation of the (4.13) with respect to the rotor displacement, $x_{c}$, and the control current, $i_{c}$, about the operating points which are typically $x_{c}=0$ and $i_{c}=0$.

$$
F=\frac{N^{2} \mu_{o} A_{p} i_{b}^{2}}{c^{3}} x_{c}+\frac{N^{2} \mu_{o} A_{p} i_{b}}{c^{2}} i_{c}=K_{p o s} x_{c}+K_{c u r} i_{c}
$$

In this paper, the following properties are utilized for MB stiffness calculation where $N=(13), \mu_{0}=12.56 e-7\left[N / A^{2}\right], A_{p}=6.7 e-4\left[m^{2}\right], c=5 e-4[m], c-x_{c}$ is air gap.

The force, position and current model represented by (4.14) applies only for a certain type of magnetic bearing. The model is included here for the sake of illustration. More complex bearings and bearing models, which include eddy currents, fringing and leakage effects are discussed in [35].

\subsubsection{Control Current Forces}

Let $K_{c u r}$ represents the current stiffness matrix. The MB control forces can then be expressed as (4.15).

$$
\begin{gathered}
\bar{F}_{C}^{B R G}=\left[\begin{array}{c}
F_{Y A}^{C} \\
F_{Y B}^{C} \\
F_{Z A}^{C} \\
F_{Z B}^{C}
\end{array}\right]=\left[\begin{array}{cccc}
K_{Y A}^{i} & 0 & 0 & 0 \\
0 & K_{Y B}^{i} & 0 & 0 \\
0 & 0 & K_{Z A}^{i} & 0 \\
0 & 0 & 0 & K_{Z B}^{i}
\end{array}\right]\left\{\begin{array}{l}
i_{Y A} \\
i_{Y B} \\
i_{Z A} \\
i_{Z B}
\end{array}\right\} \\
\text { or } \bar{F}_{C}^{B R G}=K_{c u r} \cdot i_{c}=K_{c u r} \cdot \bar{i}_{P A}
\end{gathered}
$$




\subsubsection{Transformation Matrix}

The force and moment coordinate transformation matrix from AMB coordinates to CG (rigid body) coordinates is obtained from Fig.4.9 and is given in equation (4.16)

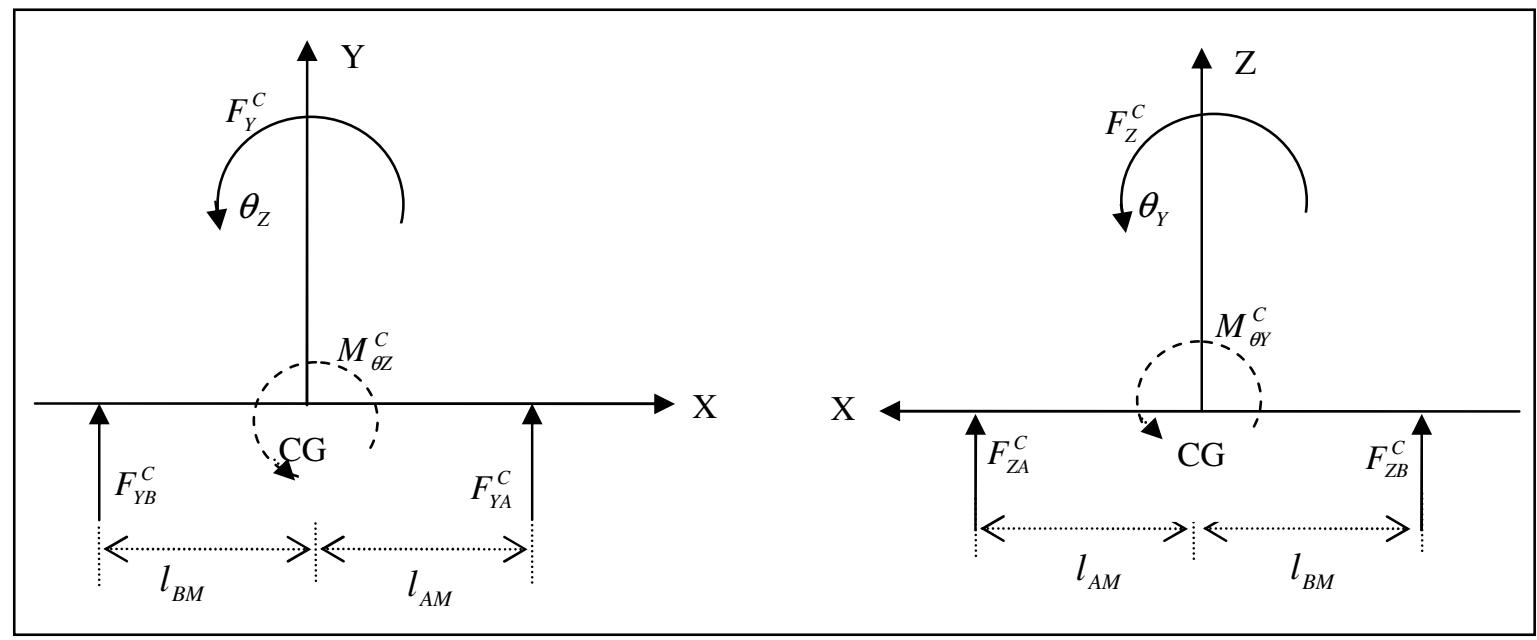

Fig. 4.9 Force - Moment Transformation Diagram

$$
\begin{aligned}
\bar{F}_{C}^{C G}=\left\{\begin{array}{c}
F_{Y}^{C} \\
M_{\theta Y}^{C} \\
F_{Z}^{C} \\
M_{\theta Z}^{C}
\end{array}\right\}= & {\left[\begin{array}{cccc}
1 & 1 & 0 & 0 \\
0 & 0 & -l_{A M} & l_{B M} \\
0 & 0 & 1 & 1 \\
l_{A M} & -l_{B M} & 0 & 0
\end{array}\right]\left\{\begin{array}{c}
F_{Y A}^{C} \\
F_{Y B}^{C} \\
F_{Z A}^{C} \\
F_{Z B}^{C}
\end{array}\right\} } \\
& \text { or } \bar{F}_{C}^{C G}=T_{B R G}^{C G} \cdot \bar{F}_{C}^{B R G}
\end{aligned}
$$

Substitute equations (4.7) and (4.15) into (4.16) to obtain the control forces in the CG (rigid body) coordinates can be shown (4.17).

$$
\bar{F}_{C}^{C G}=T_{B R G}^{C G} \cdot \bar{F}_{C}^{B R G}=\bar{T}_{B R G}^{C G} \cdot K_{c u r} \cdot \bar{K}^{P A} \cdot \bar{V}_{C T R L}
$$




\subsection{PID Control}

Magnetic suspension control laws vary widely according to the particular applications. These include both plant based versions such as $\mathrm{H}$-infinity, QR, sliding mode, etc. or variations of basic PID control. A simple PID type control is described here for sake of illustration. Filter models are included to represent the natural roll off of power amplifiers, sensors and actuators, to include effects of anti-aliasing and smoothing filters and for filter stages intentionally programmed into the feedback path for noise rejection. The parallel PID paths are shaped to suppress noise or prevent DC instability and typically have a form similar to;

$$
G_{p}(s)=\frac{1}{\tau_{p} s+1} \quad G_{I}(s)=\frac{1}{\tau_{i} s+1} \quad G_{d}(s)=\frac{s}{\left(\tau_{d} s+1\right)^{2}}
$$

For the example presented the PD controller is implemented with equal time constants $\tau_{p}$ and $\tau_{d}$ which are selected to make the cutoff frequency $\left[f_{c}=1 / 2 \pi \tau\right]$ equal to $1024[\mathrm{~Hz}]$ for both proportional and derivative paths.

\subsubsection{PD Transfer Function with Unity Gain}

The position error voltage terms are differentiated in the controller yielding a rate feedback variable as shown Fig.4.10 where $T_{\theta Z}(s)=T_{Y}(s)=T_{\theta Y}(s)=T_{Z}(s)=1 /\left(\tau_{p} s+1\right)$ and $T_{\dot{\theta} Z}(s)=T_{\dot{Y}}(s)=T_{\dot{\theta} Y}(s)=T_{\dot{Z}}(s)=s /\left(\mu_{d} s+1\right)^{2}$ in (4.18). 

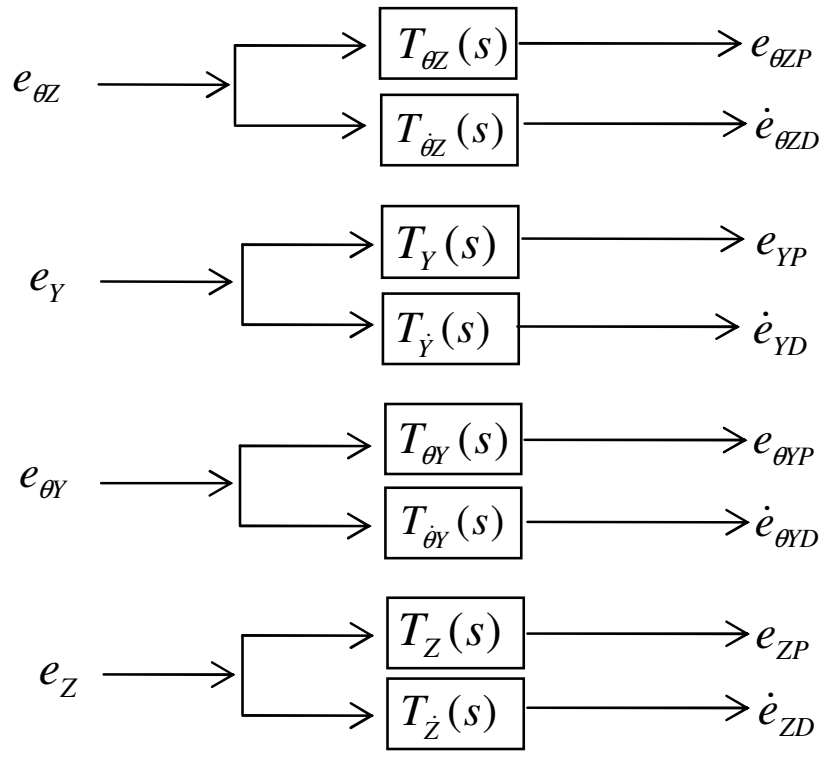

Fig. 4.10 Unity Gain PD Transfer Function Stage

\subsubsection{PD Gain Stage for MIMO (GYRO) Control}

Fig.4.11 shows the proportional, derivative and cross coupling gain stages between the tilt directions to form the MIMO, coupled controller. The cross coupled gains $\left(G_{C K \theta}\right.$ and $G_{G \theta}$ ) could play a significant role to cancel some gyroscopic moment producing high frequency forward conical pole in the case of high energy density demanding task. This gain stage is main difference between SISO and MIMO control schematic. Either $G_{C K \theta}$ or $G_{G \theta}$ is zero case, the magnetic suspension system will be unstable and effective stiffness and damping will be presented in the last section of this chapter. 


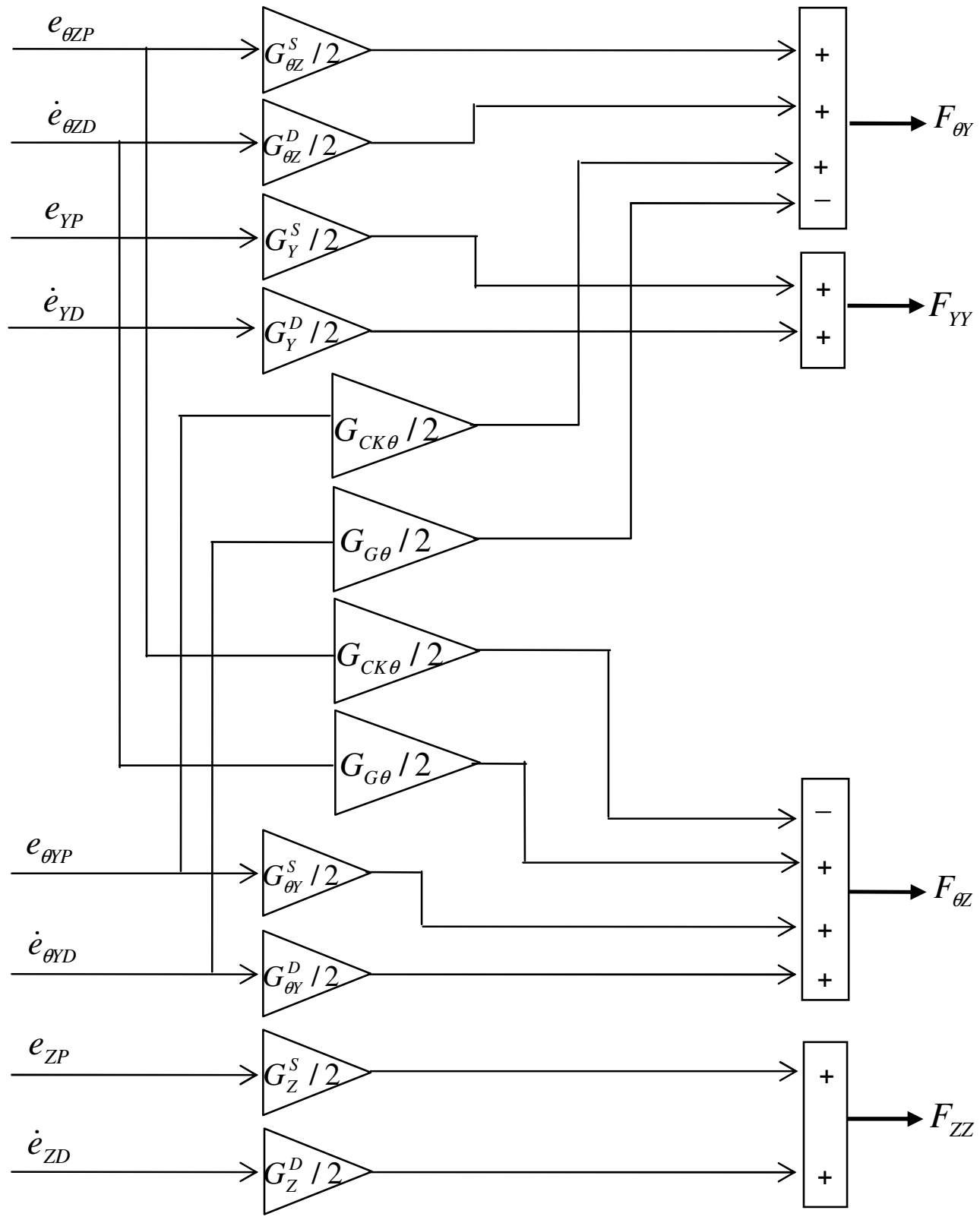

Fig. 4.11 MIMO-GYRO PD Gain Diagram 
Fig.4.11 can be represented by the matrix equation form shown (4.19)

$$
\begin{aligned}
& \bar{F}=\left(P_{C} \cdot e_{C G}^{P}+D_{C} \cdot e_{C G}^{D}\right) \\
& \text { where } P_{C}=\left[\begin{array}{cccc}
G_{Y}^{S} / 2 & 0 & 0 & 0 \\
0 & G_{C K \theta} / 2 & 0 & G_{\theta Z}^{S} / 2 \\
0 & 0 & G_{Z}^{S} / 2 & 0 \\
0 & G_{\theta Y}^{S} / 2 & 0 & -G_{C K \theta} / 2
\end{array}\right], \quad D_{C}=\left[\begin{array}{cccc}
G_{Y}^{D} / 2 & 0 & 0 & 0 \\
0 & -G_{G \theta} / 2 & 0 & G_{\theta Z}^{D} / 2 \\
0 & 0 & G_{Z}^{D} / 2 & 0 \\
0 & G_{\theta Y}^{D} / 2 & 0 & G_{G \theta} / 2
\end{array}\right] \\
& \bar{F}=\left\{\begin{array}{llll}
F_{Y Y} & F_{\theta Y} & F_{Z Z} & F_{\theta Z}
\end{array}\right\}^{T}, e_{C G}^{P}=\left\{\begin{array}{llll}
e_{Y P} & e_{\theta Y P} & e_{Z P} & e_{\theta Z P}
\end{array}\right\}^{T}, e_{C G}^{D}=\left\{\begin{array}{llll}
\dot{e}_{Y D} & \dot{e}_{\theta Y D} & \dot{e}_{Z D} & \dot{e}_{\theta Z D}
\end{array}\right\}^{T}
\end{aligned}
$$

\subsection{Output Coordinate Transformation Stage}

Fig.4.12 is diagrams for converting the rigid body coordinate control signals into the 2 pairs of orthogonal magnetic bearing actuator coordinates.
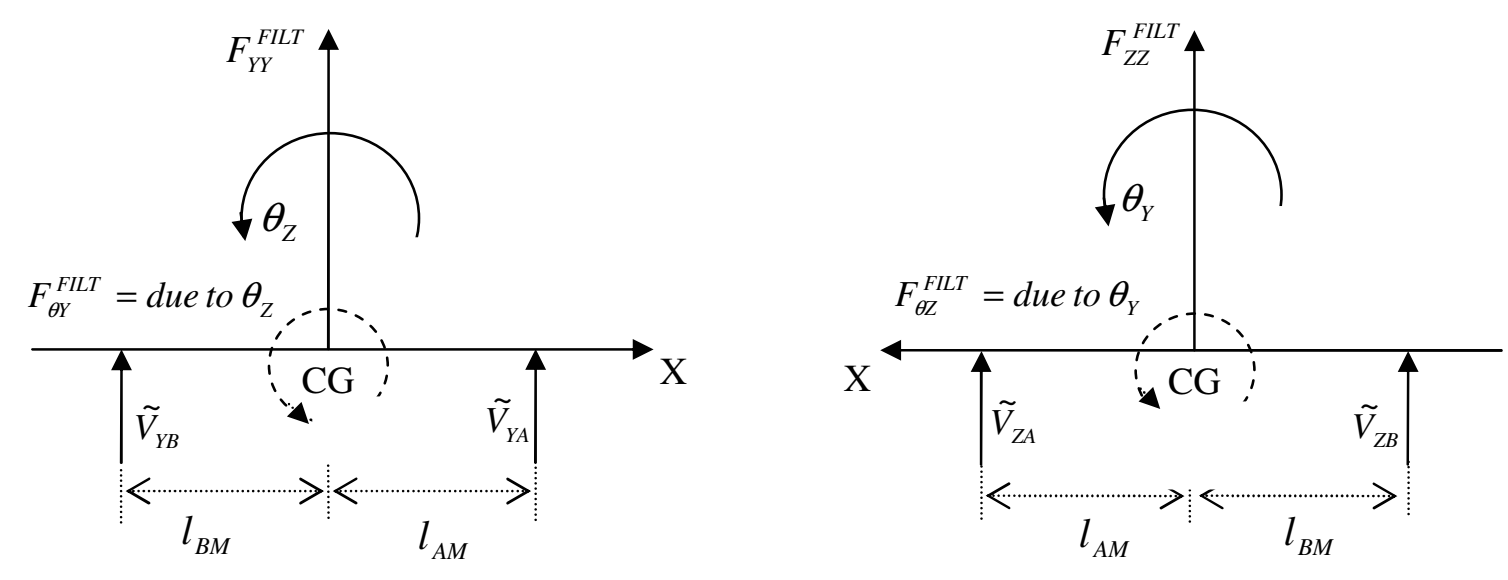

Fig. 4.12 Diagrams for Output Coordinate Transformation

Low pass filter, lead/lag compensation and notch filter stages are arranged in series at the output end of the controller. A band pass filter is also utilized to aid in canceling 
magnetic bearing forces at the spin frequency due to rotating, mass imbalance. These stages are illustrated in Fig. 4.1. The outputs of the filter stages are represented by $F_{Y Y}^{\text {Filt }}, F_{\theta Y}^{\text {Filt }}, F_{Z Z}^{\text {Filt }}$ and $F_{\theta Z}^{\text {Filt }}$, these quantities are in the rigid body coordinates and must be transformed into the magnetic bearing actuator coordinates. The transformations are provided in the following equations (4.20) and (4.21).

$$
\begin{aligned}
& F_{Y Y}^{F I L T}=\tilde{V}_{Y A}+\tilde{V}_{Y B} \\
& F_{\theta Y}^{F I L T}=l_{A M} \cdot \tilde{V}_{Y A}-l_{B M} \cdot \tilde{V}_{Y B} \\
& F_{Z Z}^{F I L T}=\tilde{V}_{Z A}+\tilde{V}_{Z B} \\
& F_{\theta Z}^{F I L T}=l_{B M} \cdot \tilde{V}_{Z B}-l_{A M} \cdot \tilde{V}_{Z A} \\
& \tilde{V}=\left\{\begin{array}{c}
\tilde{V}_{Y A} \\
\tilde{V}_{Y B} \\
\tilde{V}_{Z A} \\
\tilde{V}_{Z B}
\end{array}\right\}=\frac{1}{l_{A M}+l_{B M}}\left[\begin{array}{cccc}
l_{B M} & 1 & 0 & 0 \\
l_{A M} & -1 & 0 & 0 \\
0 & 0 & l_{B M} & -1 \\
0 & 0 & l_{A M} & 1
\end{array}\right]\left\{\begin{array}{l}
F_{Y Y}^{F I L T} \\
F_{\theta Y}^{F L T} \\
F_{Z Z}^{F L T} \\
F_{\theta Z}^{F L T}
\end{array}\right\} \text { or } \tilde{V}=T_{M F T} \cdot \bar{F}^{F I L T} \\
& \text { where } T_{M F T}=\frac{1}{l_{A M}+l_{B M}}\left[\begin{array}{cccc}
l_{B M} & 1 & 0 & 0 \\
l_{A M} & -1 & 0 & 0 \\
0 & 0 & l_{B M} & -1 \\
0 & 0 & l_{A M} & 1
\end{array}\right] \quad \text { and } \quad \bar{F}^{F L L T}=\left\{\begin{array}{l}
F_{Y Y}^{F L T} \\
F_{\theta Y}^{F L T} \\
F_{Z Z}^{F L T} \\
F_{\theta Z}^{F L T}
\end{array}\right\}
\end{aligned}
$$

The final output voltages from the controller are obtained by applying gains to compensate for differences in gains that are external to the controller, i.e. the sensors, amplifiers and magnetic bearing axes, 


$$
\bar{V}_{C T R L}=\left[\begin{array}{cccc}
\beta_{Y A} & 0 & 0 & 0 \\
0 & \beta_{Y B} & 0 & 0 \\
0 & 0 & \beta_{Z A} & 0 \\
0 & 0 & 0 & \beta_{Z B}
\end{array}\right]\left\{\begin{array}{c}
\tilde{V}_{Y A} \\
\tilde{V}_{Y B} \\
\tilde{V}_{Z A} \\
\tilde{V}_{Z B}
\end{array}\right\}=\bar{\beta}_{O U T} \cdot \tilde{V}, \quad \text { where } \quad \bar{V}_{C T R L}=\left\{\begin{array}{c}
V_{Y A} \\
V_{Y B} \\
V_{Z A} \\
V_{Z B}
\end{array}\right\}
$$

\subsection{Effective AMB Stiffness, Damping and Gyro Cancellation Torque Coefficients}

Although the AMB model presented here possesses a finite bandwidth, it is instructive to consider an infinite bandwidth approximation in order to identify equivalent stiffness, damping and gyro torque coefficients. For this ideal case, the filter output ( $\bar{F}^{F I L T}$ ) equals the filter input $(\bar{F})$. Equation (4.17) becomes

$$
\begin{aligned}
\bar{F}_{C}^{C G} & =T_{B R G}^{C G} \cdot \bar{F}_{C}^{B R G}=\bar{T}_{B R G}^{C G} \cdot K_{c u r} \cdot \bar{K}^{P A} \cdot \bar{V}_{C T R L}=\bar{T}_{B R G}^{C G} \cdot K_{c u r} \cdot \bar{K}^{P A} \cdot \bar{\beta}_{\text {OUT }} \cdot T_{M F T} \cdot \bar{F} \\
= & \bar{T}_{B R G}^{C G} \cdot\left(K_{\text {cur }} \cdot \bar{K}^{P A} \cdot \bar{\beta}_{\text {OUT }}\right) \cdot T_{M F T} \cdot\left(P_{C} \cdot e_{C G}^{P}+D_{C} \cdot e_{C G}^{D}\right) \\
& \text { Let, } \quad K_{\text {cur }} \cdot \bar{K}^{P A} \cdot \bar{\beta}_{\text {OUT }}=\left[\begin{array}{cccc}
\lambda_{Y A} & 0 & 0 & 0 \\
0 & \lambda_{Y B} & 0 & 0 \\
0 & 0 & \lambda_{\text {ZA }} & 0 \\
0 & 0 & 0 & \lambda_{\text {ZB }}
\end{array}\right]
\end{aligned}
$$

where

$$
\begin{aligned}
& \lambda_{Y A}=K_{Y A}^{i} \cdot K_{Y A}^{P A} \cdot \beta_{Y A}, \quad \lambda_{Y B}=K_{Y B}^{i} \cdot K_{Y B}^{P A} \cdot \beta_{Y B} \\
& \lambda_{Z A}=K_{Z A}^{i} \cdot K_{Z A}^{P A} \cdot \beta_{Z A}, \quad \lambda_{Z B}=K_{Z B}^{i} \cdot K_{Z B}^{P A} \cdot \beta_{Z B}
\end{aligned}
$$

Further assume that the power amplifier and current stiffness gains are identical for all axes, so $K_{j}^{i} \cdot K_{j}^{P A}$ can be expressed as $K^{i} \cdot K^{P A}$, and assume that the $\beta$ 's are tuned to make 


$$
\lambda_{Y A}=\lambda_{Y B}=\lambda_{Z A}=\lambda_{Z B}=K^{i} \cdot K^{P A}
$$

The control forces in the CG (rigid body) coordinates become:

$$
\begin{gathered}
\bar{F}_{C}^{C G}=\bar{T}_{B R G}^{C G} \cdot\left(K_{c u r} \cdot \bar{K}^{P A} \cdot \bar{\beta}_{O U T}\right) \cdot T_{M F T} \cdot\left(P_{C} \cdot e_{C G}^{P}+D_{C} \cdot e_{C G}^{D}\right) \\
=\frac{K^{i} K^{P A}}{2}\left[\begin{array}{cccc}
G_{Y}^{S} & 0 & 0 & 0 \\
0 & G_{\theta Y}^{S} & 0 & -G_{C K \theta} \\
0 & 0 & G_{Z}^{S} & 0 \\
0 & G_{C K \theta} & 0 & G_{\theta Z}^{S}
\end{array}\right]\left\{\begin{array}{c}
e_{Y} \\
e_{\theta Y} \\
e_{Z} \\
e_{\theta Z}
\end{array}\right\}+\frac{K^{i} K^{P A}}{2}\left[\begin{array}{cccc}
G_{Y}^{D} & 0 & 0 & 0 \\
0 & G_{\theta Y}^{D} & 0 & G_{G \theta} \\
0 & 0 & G_{Z}^{D} & 0 \\
0 & -G_{G \theta} & 0 & G_{\theta Z}^{D}
\end{array}\right]\left\{\begin{array}{c}
\dot{e}_{Y} \\
\dot{e}_{\theta Y} \\
\dot{e}_{Z} \\
\dot{e}_{\theta Z}
\end{array}\right\}
\end{gathered}
$$

The active stiffness and damping in the CG coordinates are then obtained by comparing (4.17) with (4.26), yielding

$$
\begin{gathered}
F_{Y}^{C}=m_{f} \ddot{e}_{Y}=\frac{K^{i} K^{P A}}{2}\left\{G_{Y}^{S} \cdot e_{Y}+G_{Y}^{D} \cdot \dot{e}_{Y}\right\} \\
M_{\theta Y}^{C}=I_{T} \ddot{e}_{\theta Y}+I_{P} \omega_{f} \dot{e}_{\theta Z}=\frac{K^{i} K^{P A}}{2}\left\{G_{\theta Y}^{S} \cdot e_{\theta Y}-G_{C K \theta} \cdot e_{\theta Z}+G_{\theta Y}^{D} \cdot \dot{e}_{\theta Y}+G_{G \theta} \cdot \dot{e}_{\theta Z}\right\} \\
F_{Z}^{C}=m_{f} \ddot{e}_{Z}=\frac{K^{i} K^{P A}}{2}\left\{G_{Z}^{S} \cdot e_{Z}+G_{Z}^{D} \cdot \dot{e}_{Z}\right\} \\
M_{\theta Z}^{C}=I_{T} \ddot{e}_{\theta Z}-I_{P} \omega_{f} \dot{e}_{\theta Y}=\frac{K^{i} K^{P A}}{2}\left\{G_{\theta Z}^{S} \cdot e_{\theta Z}+G_{C K \theta} \cdot e_{\theta Y}+G_{\theta Z}^{D} \cdot \dot{e}_{\theta Z}-G_{G \theta} \cdot \dot{e}_{\theta Y}\right\}
\end{gathered}
$$

Therefore, for the ideal, non-saturated and infinite bandwidth case, the effective stiffness and damping matrices are:

$$
\bar{K}_{\infty}^{e f f}=\left[\begin{array}{cccc}
K_{Y, Y} & K_{Y, \theta Y} & K_{Y, Z} & K_{Y, \theta Z} \\
K_{\theta Y, Y} & K_{\theta Y, \theta Y} & K_{\theta Y, Z} & K_{\theta Y, \theta Z} \\
K_{Z, Y} & K_{Z, \theta Y} & K_{Z, Z} & K_{Z, \theta Z} \\
K_{\theta Z, Y} & K_{\theta Z, \theta Y} & K_{\theta Z, Z} & K_{\theta Z, \theta Z}
\end{array}\right]=\frac{K^{i} K^{P A}}{2}\left[\begin{array}{cccc}
G_{Y}^{S} & 0 & 0 & 0 \\
0 & G_{\theta Y}^{S} & 0 & -G_{C K \theta} \\
0 & 0 & G_{Z}^{S} & 0 \\
0 & G_{C K \theta} & 0 & G_{\theta Z}^{S}
\end{array}\right]
$$




$$
\bar{C}_{\infty}^{e f f}=\left[\begin{array}{cccc}
C_{Y, Y} & C_{Y, \theta Y} & C_{Y, Z} & C_{Y, \theta Z} \\
C_{\theta Y, Y} & C_{\theta Y, \theta Y} & C_{\theta Y, Z} & C_{\theta Y, \theta Z} \\
C_{Z, Y} & C_{Z, \theta Y} & C_{Z, Z} & C_{Z, \theta Z} \\
C_{\theta Z, Y} & C_{\theta Z, \theta Y} & C_{\theta Z, Z} & C_{\theta Z, \theta Z}
\end{array}\right]=\frac{K^{i} K^{P A}}{2}\left[\begin{array}{cccc}
G_{Y}^{D} & 0 & 0 & 0 \\
0 & G_{\theta Y}^{D} & 0 & G_{G \theta} \\
0 & 0 & G_{Z}^{D} & 0 \\
0 & -G_{G \theta} & 0 & G_{\theta Z}^{D}
\end{array}\right]
$$

Equations (4.27) to (4.30) may be inverted to solve for the required MIMO gains in terms of the required natural frequencies and damping ratios as

(1) “CG” control gains:

$$
\begin{aligned}
& G_{Y}^{S}=\frac{2 m_{f} \omega_{c y l}^{2}}{K^{i} K^{P A}}, G_{Y}^{D}=\frac{4 m_{f} \zeta_{c y l} \omega_{c y l}}{K^{i} K^{P A}}, G_{\theta Y}^{S}=\frac{2 I_{T} \omega_{c o n}^{2}}{K^{i} K^{P A}}, G_{\theta Y}^{D}=\frac{4 I_{T} \zeta_{c o n} \omega_{c o n}}{K^{i} K^{P A}} \\
& G_{Z}^{S}=G_{Y}^{S}, G_{Z}^{D}=G_{Y}^{D}, G_{Z}^{D}=G_{Y}^{D} \text { and } G_{\theta Z}^{D}=G_{\theta Y}^{D}
\end{aligned}
$$

\section{(2) Gyro control gains}

$$
G_{G \theta}=\frac{2 \cdot I_{P} \cdot \omega_{s p i n}}{K^{i} K^{P A}}, G_{C K \theta}=0.5 \cdot G_{\theta Y}^{S}
$$

The gyro control gains, $G_{G \theta}$ and $G_{C K \theta}$, are positioned in equation (4.26) to buck (cancel) a portion of the natural gyroscopic moments that result from spinning the flywheel. 


\section{CHAPTER V}

\section{VIBRATION CONTROL OF FLEXIBLE APPENDAGES AND FLYWHEEL UNBALANCE ISOLATION*}

\subsection{Overview}

This chapter presented the methods that reduce flexible satellite appendage's oscillation and flywheel imbalance force transmitting to satellite. The vibration control mass (VCM) is placed on the ends of each appendage to demonstrate its effects. The notch filter and band-pass filter stages are also analyzed in this chapter. The imbalance force due to unbalanced mass could be reduced by positioning the center frequency of a notch filter at the flywheel spin speed in the feedback path of the magnetic bearing supported system, however, there still exists another components of force which created by position stiffness and flywheel relative motion. Section 5.3 will discuss about more details how to eliminate the residual magnetic bearing dynamic forces.

\subsection{Vibration Control Mass (VCM) to Suppress the Oscillation of the Satellite's Flexible Appendages}

A machine or system may experience excessive vibration if it is acted upon by a force whose excitation frequency nearly coincides with a natural frequency of the machine or system. In such case, the vibration of the machine or system can be reduced by utilizing a dynamic vibration absorber [36].

\footnotetext{
*Reprinted with permission from "MIMO Active Vibration Control of Magnetically Suspended Flywheels for Satellite IPAC Service," Park, J., 2007, Journal of Dynamic Systems, Measurement and Control, Accepted, Copyright [2008] by ASME.
} 


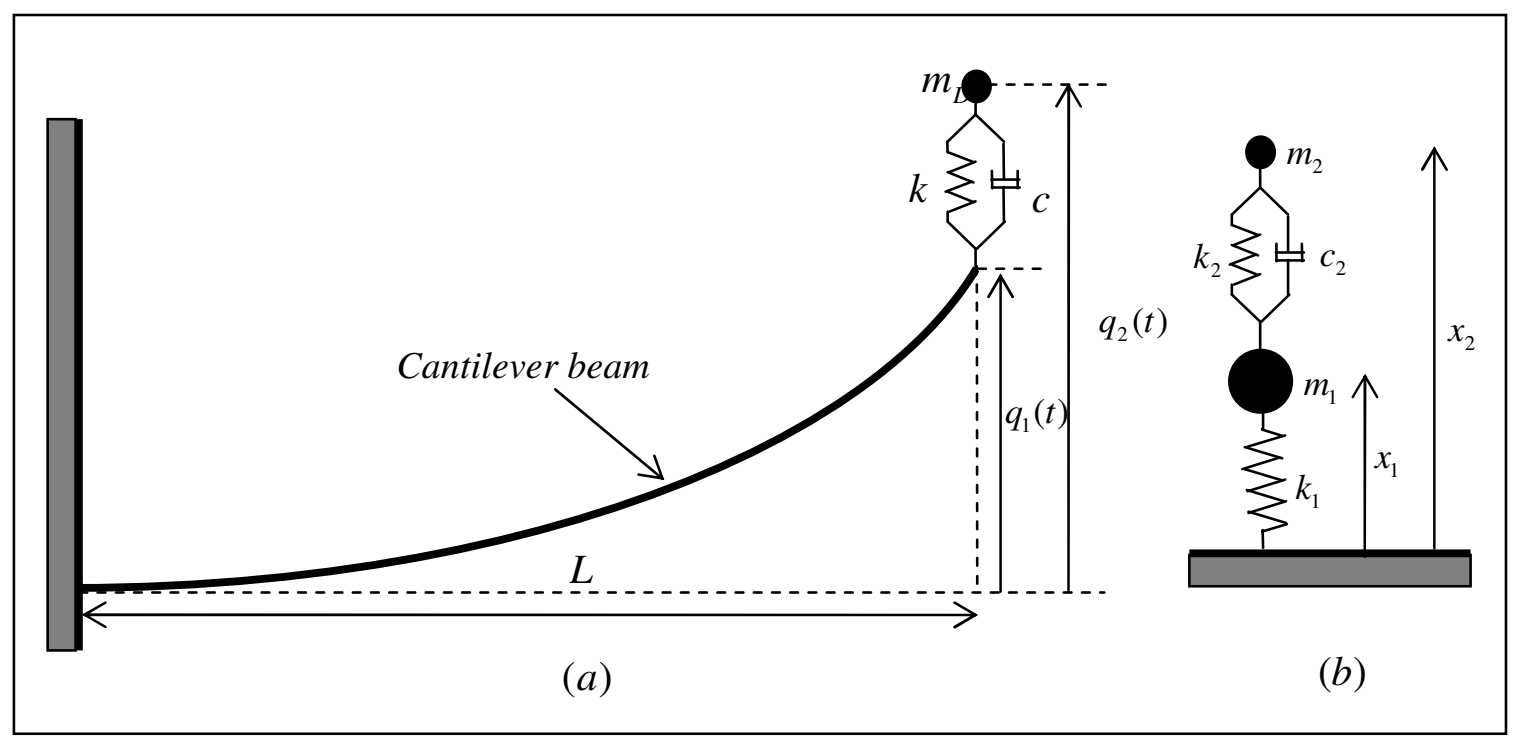

Fig. 5.1 Vibration Control Mass (VCM) Model

For the classical vibration absorber case, VCM approach in the Fig.5.1(a) can be modeled as Fig.5.1(b). The equations of motions, steady-state solutions and more analytical parts are given by [36]. The optimum damping ratio can be calculated as,

$$
\xi_{\text {optimum }}^{2}=\frac{3 \mu}{8(1+\mu)^{3}}
$$

where $\mu=m_{2} / m_{1}$ from Fig.5.1(b) [36]. In the case of $1.35[\mathrm{~kg}$ ] vibration control mass, the mass ratio $(\mu)=0.3$ and $\xi_{\text {optimum }}=0.2236$ which is similar with in the Table.5.1.

For illustration of VCM approach, assume that a flexible appendage behaves similar to a cantilever beam with deflection pattern,

$$
y(x, t)=\left(\frac{x}{L}\right)^{2} \cdot q_{1}(t)
$$

where $q_{1}(t)$ is a generalized coordinate for the beam's deflection. The equations of 
motion for the VCM in Fig 5.1(a) and the beam coordinate becomes,

$$
\begin{gathered}
{\left[\begin{array}{cc}
\frac{\rho A L}{5} & 0 \\
0 & m_{D}
\end{array}\right]\left\{\begin{array}{l}
\ddot{q}_{1} \\
\ddot{q}_{2}
\end{array}\right\}+\left[\begin{array}{cc}
c & -c \\
-c & c
\end{array}\right]\left\{\begin{array}{l}
\dot{q}_{1} \\
\dot{q}_{2}
\end{array}\right\}+\left[\begin{array}{cc}
\frac{4 E I}{L^{3}}+k & -k \\
-k & k
\end{array}\right]\left\{\begin{array}{l}
q_{1} \\
q_{2}
\end{array}\right\}=\left[\begin{array}{l}
0 \\
0
\end{array}\right]} \\
\text { or } M \ddot{q}+C \dot{q}+K q=0
\end{gathered}
$$

where, $q=\left\{\begin{array}{l}q_{1} \\ q_{2}\end{array}\right\}, \quad M=\left[\begin{array}{cc}\frac{\rho A L}{5} & 0 \\ 0 & m_{D}\end{array}\right], \quad C=\left[\begin{array}{cc}c & -c \\ -c & c\end{array}\right], \quad K=\left[\begin{array}{cc}\frac{4 E I}{L^{3}}+k & -k \\ -k & k\end{array}\right]$

The first order form of this equation can be written as

$$
\dot{X}=A X \text { where } A=\left[\begin{array}{cc}
0 & I_{2} \\
-M^{-1} K & -M^{-1} C
\end{array}\right] \text { and } X=\left\{\begin{array}{c}
q \\
\dot{q}
\end{array}\right\}
$$

The damping ratios $\left(\xi_{i}\right)$ may then be obtained from the eigenvalues of $A$ as

$$
\left\{\xi_{i}=\frac{\text { abs }\left[(\text { real part of eigenvalue })_{i}\right]}{\text { abs }\left[\left(\text { eigenvalue }_{i}\right]\right.}, i=1,2,3,4\right\}
$$

The VCM's attachment stiffness and damping were selected to maximize the $1^{\text {st }}$ mode's damping ratio as illustrated in Table 5.1. 
Table 5.1 VCM Damping Ratios VS VCM Attachment Stiffness and Damping

\begin{tabular}{cccccccc}
\hline \hline$m_{D}[\mathrm{~kg}]$ & $c_{\text {opt }}[\mathrm{Ns} / \mathrm{m}]$ & $k_{\text {opt }}[\mathrm{N} / \mathrm{m}]$ & $\xi_{1}$ & $\xi_{2}$ & $\xi_{3}$ & $\xi_{4}$ & $\xi_{\min }$ \\
\hline 0.45 & 0.9065 & 10.767 & 0.1136 & 0.1136 & 0.1398 & 0.1398 & 0.1136 \\
1.35 & 6.1197 & 32.310 & 0.2757 & 0.2757 & 0.2491 & 0.2491 & 0.2491 \\
2.25 & 10.202 & 39.672 & 0.3240 & 0.3240 & 0.3286 & 0.3286 & 0.3240 \\
3.15 & 14.280 & 43.575 & 0.3950 & 0.3950 & 0.3878 & 0.3878 & 0.3878 \\
4.05 & 18.370 & 45.900 & 0.4888 & 0.4888 & 0.43 & 0.43 & 0.43 \\
\hline \hline
\end{tabular}

\subsection{Flywheel Unbalance Isolation}

Mass imbalance of the flywheel creates a force at its spin frequency, which in turn causes a time varying error in the magnetic suspension position control at the spin frequency. This may be very undesirable since the ensuing vibrations can interfere with the proper operation of onboard, precision instrumentation. This may be rectified by positioning the center frequency of a notch filter at the flywheel spin frequency in the feedback path of the magnetic suspension control. Equation (4.14) shows that there still exists another component of force at the spin frequency due to the position stiffness and flywheel relative displacement. This force is proportional to the relative vibration of the rotor with respect to the stator, and so the force may be cancelled by band-pass filtering this relative vibration at the spin frequency, and then multiplying this signal by an appropriate gain to create forces that opposes the position stiffness related forces. The characteristic of notch and band-pass filters are presented in the Fig.5.2, and the transfer function of standard second order notch filter is shown in the Fig.5.3. It could be 
implemented to eliminate sensor runout disturbance with its own characteristic which reduces the input signal around a specific frequency and its characteristic is determined by the center frequency of the filter (flywheel spin speed) and $Q$ factor.

The band-pass filter is modeled as a second-order transfer function as shown in Fig.5.3. It is a filter that passes frequencies within a specific range and rejects frequencies outside of that range. The Fig.5.2 utilized that $Q=50$ for notch and band-pass filter, $k=1$ for band-pass filter and flywheel spin speed is $40000[\mathrm{rpm}]$. In addition, a low pass filter is utilized in the flywheel motor torque feedback loop to remove high frequency components.

(a) Characteristic of Band Pass Filter

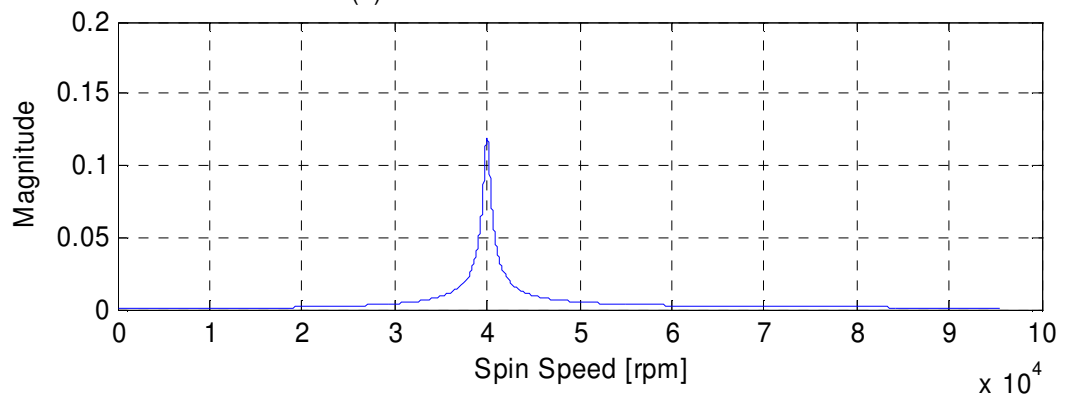

(b) Characteristic of Notch filter

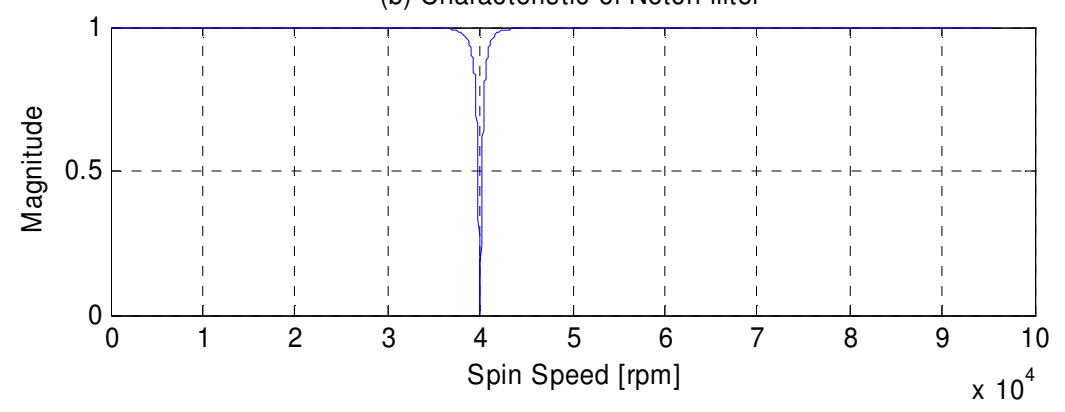

Fig. 5.2 Characteristics of Band-Pass and Notch Filters 


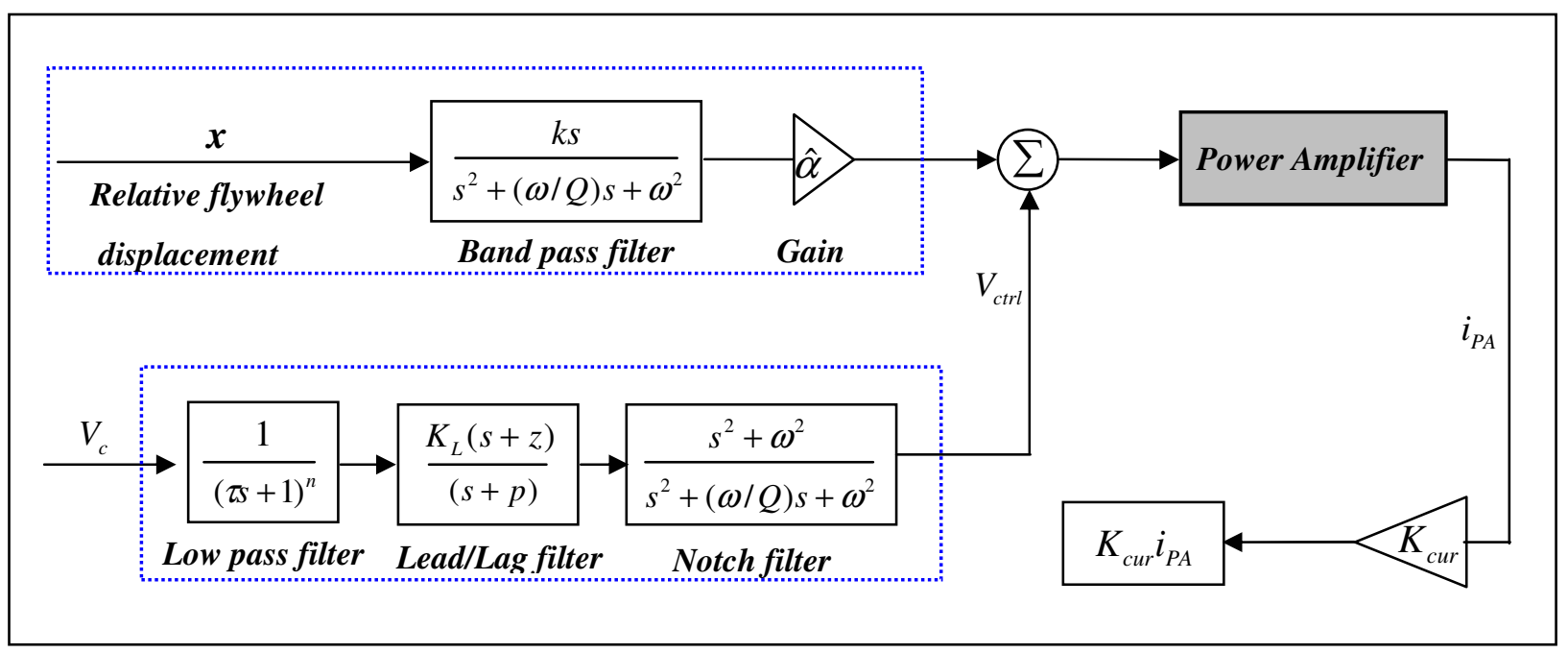

Fig. 5.3 AMB Control to Attenuate the Forces at the Spin Frequency

The output amplitude of the band pass filter at the flywheel spin speed is $Q \cdot x / \omega$, where $Q$ is the band-pass quality factor, $\omega$ is $40000[\mathrm{rpm}]$ and $x$ represents the relative vibration (displacement) at the magnetic bearing actuator location. The total magnetic bearing force at the spin frequency is expressed as $F_{M B}=K_{p o s} \cdot x+K_{c u r} \cdot i_{P A}$. The forces applied to the satellite by the magnetic bearing actuator, at the flywheel spin frequency, will therefore be null if it is assumed that the power amplifier gain is $1[A / V]$ and the gain $\hat{\alpha}$ in Fig.5.3 is selected as $\hat{\alpha}=-\frac{K_{p o s}}{K_{\text {cur }}} \cdot \frac{\omega}{Q}$. 


\section{CHAPTER VI}

\section{NUMERICAL SIMULATIONS*}

\subsection{Overview}

The numerical example results are presented in the Chapter VI. This chapter has mainly separated 7 sections. The satellite including flexible appendages and their finite element model are shown in the section 6.2. As far as total system coordinate concerned, inertial reference and satellite body coordinates are described in the Chapter II and the rests of coordinates (flywheel, appendage and each housing reference) are shown in this chapter. Also, the system parameter values for the numerical results are tabulated. The validation of finite element model for flexibility is presented in the section 6.3 and compared analytical solution with finite element model results. The satellite reference motion and responses including $\mathrm{MB}$ suspension and flexibility are discussed in the section 6.4. In the section 6.5 and 6.6, MIMO active magnetic bearing suspension system results are compared with SISO control case and vibration control mass effects on flywheels and flexible appendages motions are shown, respectively. The last section is about isolation of the satellite from flywheel's mass imbalance forces. Notch and band-pass filters are utilized to reduce transmitted force to satellite.

The present simulation model comprised with two different types of feedback controller. The first one is the flywheel motor toque control for satellite attitude control

\footnotetext{
*Reprinted with permission from "MIMO Active Vibration Control of Magnetically Suspended Flywheels for Satellite IPAC Service," Park, J., 2007, Journal of Dynamic Systems, Measurement and Control, Accepted, Copyright [2008] by ASME.
} 
and power transfer and the other is magnetically suspended flywheel position control. Fig. 6.1 explains the details of these two feedback control loop. FB1 and FB2 indicate that satellite attitude and power transfer feedback control loop, respectively. FB3 shows the flywheel position feedback control loop. $\mathrm{AC} 1$ and $\mathrm{AC} 2$ are motor torque applied to flywheel and MB actuator acting on the flywheel, respectively. AC3 is satellite solar power charging, whenever the satellite towards to sun, it stores excessive energy using either chemical battery or flywheel energy storage system. TG is like supervisor to command target motions.

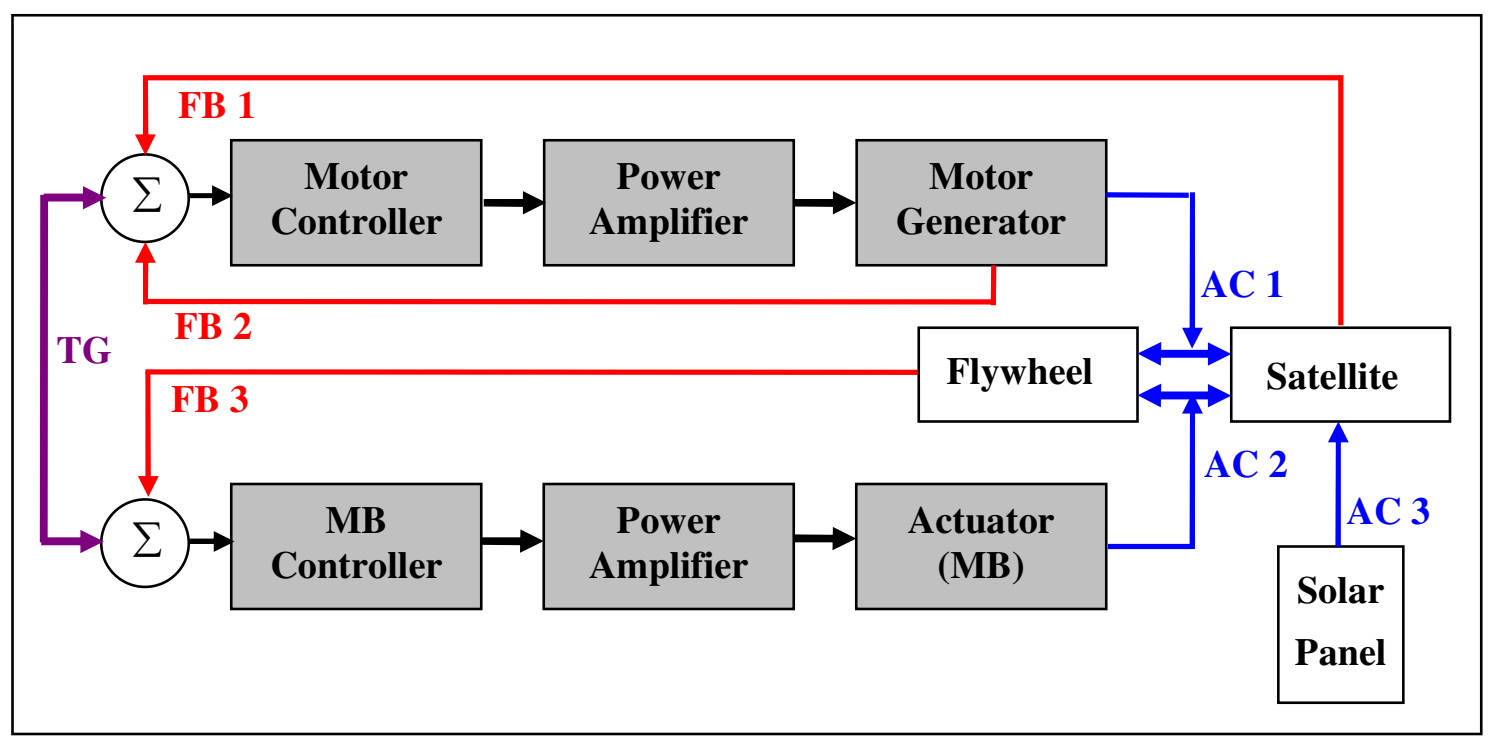

Fig. 6.1 IPAC System Feedback Control Loop 


\subsection{Model Description}

The overall configuration of this example employs four rigid flywheels aligned in a tetrahedral shaped array and two flexible appendages attached to the satellite in the Fig.6.2 [18]. The mass centers of the flywheels and appendages are offset from the satellite mass center by distances $R_{i}$ and $A_{i}$, respectively. Each flywheel's housing is assumed to be rigid and have a rigid attachment to the satellite. Each appendage's motions are referenced to a fictitious rigid "appendage reference" which coincides with the appendage centerline in the zero motion state as depicted in Fig.6.3. Fig.6.2 indicates that the flywheel housing body coordinate axes, $\hat{h}_{f, 3}$, of module 1,2 and 3 are separated by 120 degrees from each other, and their spin axes make a 19.471[deg] angle with respect to the satellite $\hat{S}_{1}, \hat{S}_{2}$ plane. Module 4 is perpendicular to this plane and pointed along the $-\hat{S}_{3}$ axis direction. All flywheel spin directions are in the clockwise sense as viewed from the satellite coordinate origin. It is assumed that the satellite inertias include the effects of the flywheel housings and that the MB centers coincide with the flywheel housing center lines. 


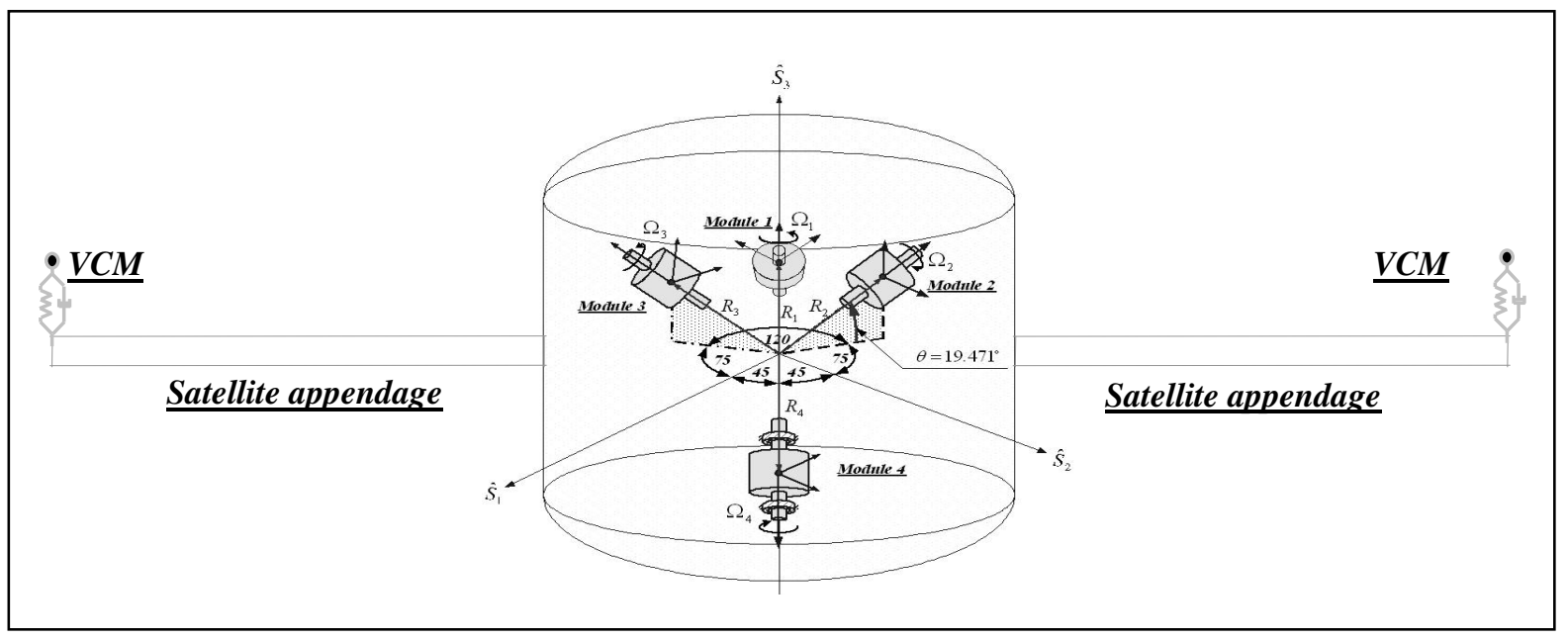

Fig. 6.2 Tetrahedral Array of Flywheels Attached to the Satellite

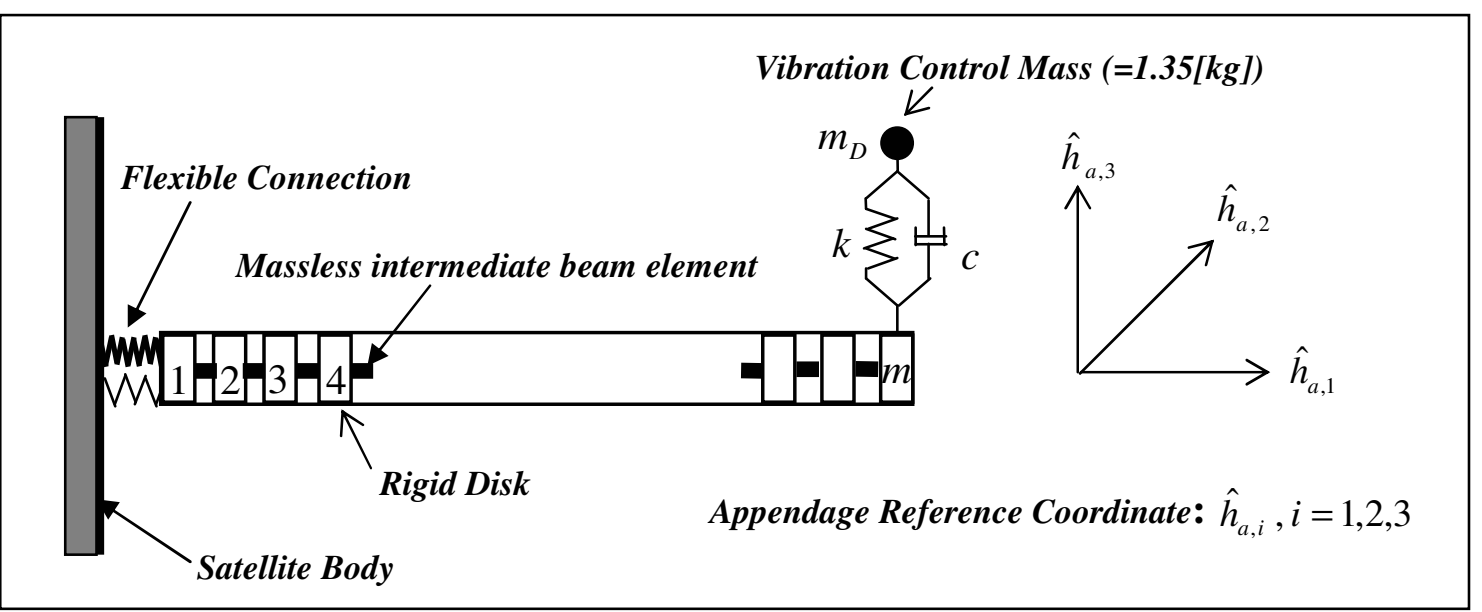

Fig. 6.3 Flexible Appendage Model Consisting of Beam Type Elements

The 1.35[kg] (3[lb]) VCM shown in Fig.6.3 is utilized to reduce the vibration of the appendage thereby reducing ripple error in the power transfer (charge or discharge) and suppress the oscillation of flexible appendage. The detailed VCM effects are discussed and plotted in the section 6.6. This mass is attached to the free end of the appendage 
utilizing a spring and damper and is constrained in the model to displace only perpendicular to the appendage. The appendage model also includes a small level of structural damping to more closely simulate an actual structure. An actual appendage on a satellite may be collapsible and consist of a truss-like structure with embedded masses and panels. The low stiffness and natural frequencies of this form of appendage is emulated by assigning a low value of Young's modulus for the appendages, which are otherwise modeled as uniform cantilever beams of rectangular cross-section.

Numerical simulation system model parameters are presented in the Table 6.1 and AMB parameters and their control gains are tabulated in the Table 6.2 and 6.3, respectively.

Table 6.1 Model Parameter Values

\begin{tabular}{|c|c|c|c|c|c|c|}
\hline Parameters & Weight & $I_{P} \cdot g$ & $I_{T} \cdot g$ & $\begin{array}{l}\text { Initial } \\
\text { speed }\end{array}$ & $R_{f}$ & $R_{a}$ \\
\hline Satellite & $\begin{aligned} & 1360[\mathrm{~kg}] \\
= & 3000[\mathrm{lb}]\end{aligned}$ & $\begin{aligned} & 1693\left[\mathrm{~N}-\mathrm{m}-\mathrm{s}^{2}\right] \\
= & 15000\left[\mathrm{lb}-\mathrm{in}-\mathrm{s}^{2}\right]\end{aligned}$ & $\begin{aligned} & 2258\left[\mathrm{~N}-\mathrm{m}-\mathrm{s}^{2}\right] \\
= & 20000\left[\mathrm{lb}-\mathrm{in}-\mathrm{s}^{2}\right]\end{aligned}$ & 0 & & \\
\hline Flywheel & $\begin{array}{l}22.7[\mathrm{~kg}] \\
=50[\mathrm{lb}]\end{array}$ & $\begin{aligned} & 69.5\left[\mathrm{~N}-\mathrm{m}-\mathrm{s}^{2}\right] \\
= & 617\left[\mathrm{lb}-\mathrm{in}-\mathrm{s}^{2}\right]\end{aligned}$ & $\begin{array}{l}55.67\left[\mathrm{~N}-\mathrm{m}-\mathrm{s}^{2}\right] \\
=494\left[\mathrm{lb}-\mathrm{in}-\mathrm{s}^{2}\right]\end{array}$ & $\begin{array}{r}40,000 \\
{[\mathrm{rpm}]}\end{array}$ & $\begin{array}{l}0.61[\mathrm{~m}] \\
=24[\mathrm{in}]\end{array}$ & \\
\hline Appendage & $\begin{array}{l}22.7[\mathrm{~kg}] \\
=50[\mathrm{lb}]\end{array}$ & & & 0 & & $\begin{array}{l}2.4[\mathrm{~m}] \\
=95[\mathrm{in}]\end{array}$ \\
\hline
\end{tabular}

Note: $1 . * *: \operatorname{Ia}=\operatorname{diag}\left(0.47,18.4,18.85\left[N-m-s^{2}\right]\right)=\operatorname{diag}\left(4.178,162.7,167\left[l b-i n-s^{2}\right]\right)$

2. Young's modulus and shear modulus of flexible appendages: $E=2.06 \times 10^{9}\left[\mathrm{~N} / \mathrm{m}^{2}\right], G=8.27 \times 10^{10}\left[\mathrm{~N} / \mathrm{m}^{2}\right]$

3. Length of each appendage $=3.2[\mathrm{~m}]$ 
Table 6.2 AMB Parameter Values

\begin{tabular}{ccccc}
\hline \hline $\begin{array}{c}\text { Magnetic } \\
\text { Bearing }\end{array}$ & $\begin{array}{c}\text { Current Stiffness } \\
K_{c u r}\end{array}$ & $\begin{array}{c}\text { Position Stiffness } \\
K_{p o s}\end{array}$ & $\begin{array}{c}\text { Load } \\
\text { Capacity }\end{array}$ & $\begin{array}{c}\text { Locations from } \\
\text { flywheel CG }\end{array}$ \\
\hline \multirow{2}{*}{ Combo (Radial) } & $41.4[\mathrm{~N} / \mathrm{A}]$ & $-1208312[\mathrm{~N} / \mathrm{m}]$ & $444.8[\mathrm{~N}]$ & $l_{m b}=0.127[\mathrm{~m}]$ \\
& $=9.3[\mathrm{lb} / \mathrm{A}]$ & $=-6900[\mathrm{~b} / \mathrm{in}]$ & $=100[\mathrm{lb}]$ & $=5[\mathrm{in}]$ \\
Combo (Axial) & $85.5[\mathrm{~N} / \mathrm{A}]$ & $-1383448[\mathrm{~N} / \mathrm{m}]$ & $889.6[\mathrm{~N}]$ & $l_{m b}=0.127[\mathrm{~m}]$ \\
& $=19[\mathrm{lb} / \mathrm{A}]$ & $=-7900[\mathrm{lb} / \mathrm{in}]$ & $=200[\mathrm{lb}]$ & $=5[\mathrm{in}]$ \\
& $39.1[\mathrm{~N} / \mathrm{A}]$ & $-1078739[\mathrm{~N} / \mathrm{m}]$ & $444.8[\mathrm{~N}]$ & $l_{m b}=0.127[\mathrm{~m}]$ \\
Radial & $=8.8[\mathrm{lb} / \mathrm{A}]$ & $=-6160[\mathrm{lb} / \mathrm{in}]$ & $=100[\mathrm{lb}]$ & $=5[\mathrm{in}]$ \\
\hline \hline
\end{tabular}

Table 6.3 AMB and Flywheel Motor Control Gains

\begin{tabular}{cccccccccccc}
\hline \hline \multicolumn{11}{c}{ MIMO Control Gains } & \\
\hline$G_{Y}^{S}$ & $G_{Y}^{D}$ & $G_{\theta Y}^{S}$ & $G_{\theta Y}^{D}$ & $G_{G \theta}$ & $G_{Z}^{S}$ & $G_{Z}^{D}$ & $G_{\theta Z}^{S}$ & $G_{\theta Z}^{D}$ & $G_{C K \theta}$ & $k_{1}$ & $k_{2}$ \\
11.1 & 0.012 & 324 & 0.064 & 0.67 & 11.1 & 0.012 & 324 & 0.064 & 162 & 15.4 & 117 \\
\hline
\end{tabular}

\subsection{Validation of Finite Element Model for Satellite Flexibility}

The Finite Element Model described in the Fig.2.3 is validated in the section. The purpose of this section is that comparing a simple illustration of gyroscopic effects, forward and backward eigenvalues, synchronous whirl analysis between the long rigid rotor case and its finite element model case. Fig. 6.4 shows the model, in which the disk may be a long cylinder. The origin of the nonrotating XYZ axes is at the undeflected centroid of the rotor. The two bearings are located at $X=L / 2$. The undamped eigenvalues 
can be found by substituting a purely elastic and symmetric model for the bearing forces into (6.1) thru (6.4) [37]

$$
\begin{aligned}
& m \ddot{Y}+2 K_{L} Y=0 \\
& m \ddot{Z}+2 K_{L} Z=0 \\
& I_{T} \ddot{\beta}+I_{P} \omega_{s} \dot{\alpha}+\frac{1}{2} K_{L} L^{2} \beta=0 \\
& I_{T} \ddot{\alpha}-I_{P} \omega_{s} \dot{\beta}+\frac{1}{2} K_{L} L^{2} \alpha=0
\end{aligned}
$$

The characteristic matrix resulting from the homogeneous solution $a_{j} e^{s t}$, for $j=1,2$,

3,4 is [37]

$$
\left[\begin{array}{cccc}
\left(m s^{2}+2 K_{L}\right) & 0 & 0 & 0 \\
0 & \left(m s^{2}+2 K_{L}\right) & 0 & 0 \\
0 & 0 & I_{T} s^{2}+K_{L} L^{2} / 2 & I_{P} \omega_{s} \\
0 & 0 & -I_{P} \omega_{s} & I_{T} s^{2}+K_{L} L^{2} / 2
\end{array}\right]\left\{\begin{array}{l}
a_{1} \\
a_{2} \\
a_{3} \\
a_{4}
\end{array}\right\}=\left\{\begin{array}{l}
0 \\
0 \\
0 \\
0
\end{array}\right\}
$$

The purely imaginary eigenvalues of the system are $s_{j}= \pm i \omega_{j}$, for $j=1,2,3,4$, where

$$
\begin{aligned}
& \omega_{1}=\omega_{2}=\sqrt{2 K_{L} / m} \\
& \omega_{3}=\frac{I_{P}}{2 I_{T}} \omega_{s}+\sqrt{\frac{K_{L} L^{2}}{2 I_{T}}+\left(\frac{I_{P}}{2 I_{T}} \omega_{s}\right)^{2}} \\
& \omega_{4}=\frac{I_{P}}{2 I_{T}} \omega_{s}-\sqrt{\frac{K_{L} L^{2}}{2 I_{T}}+\left(\frac{I_{P}}{2 I_{T}} \omega_{s}\right)^{2}}
\end{aligned}
$$

These are undamped natural frequencies of the rotor-bearing system, if the rotor 
angular spin speed $\omega_{s}$ is zero, the natural frequencies are $\sqrt{2 K_{L} / m}$ and $\sqrt{K_{L} L^{2} / 2 I_{T}}$. In the case, the vibration modes are heaving-swaying and pitching-yawing, respectively. A nonzero shaft spin speed $\omega_{s}$ changes $\omega_{3}$ and $\omega_{4}$ frequencies but not $\omega_{1}$ and $\omega_{2}$.

The latter are the natural frequencies of cylindrical whirl. Shaft spin speed $\omega_{s}$ raises the $\omega_{3}$ frequency above the planar pitching vibration value $\sqrt{K_{L} L^{2} / 2 I_{T}}$ and lower $\omega_{4}$ frequency. These are the natural frequencies of forward and backward conical whirl.

The axial and torsional springs are attached to the each end of rotor-bearing model to compare finite element analysis results. The equation (6.9) and (6.10) indicate the axial and torsional natural frequencies, respectively, and Table 6.4 shows the long rigid rotorbearing model parameters utilized in this section. The finite element model of Fig.6.4 is comprised of 6 elements ( 7 nodes) and each node executes 6 degrees of freedom motions.

$$
\begin{gathered}
\omega_{5}=\sqrt{2 K_{A} / m} \\
\omega_{6}=\sqrt{2 K_{T} / I_{P}}
\end{gathered}
$$

Table 6.5 shows that frequencies results obtained from analytical and finite element model. The natural frequencies are almost identical except for the conical whirl mode, $\omega_{3}$, which has about $0.5[\%]$ differences between analytical solution and finite element model. One of conical whirl frequency, $\omega_{4}$, will converge to zero when spin speed is very high. Table 6.5 doesn't show $\omega_{4}$ which has negative frequency value. 


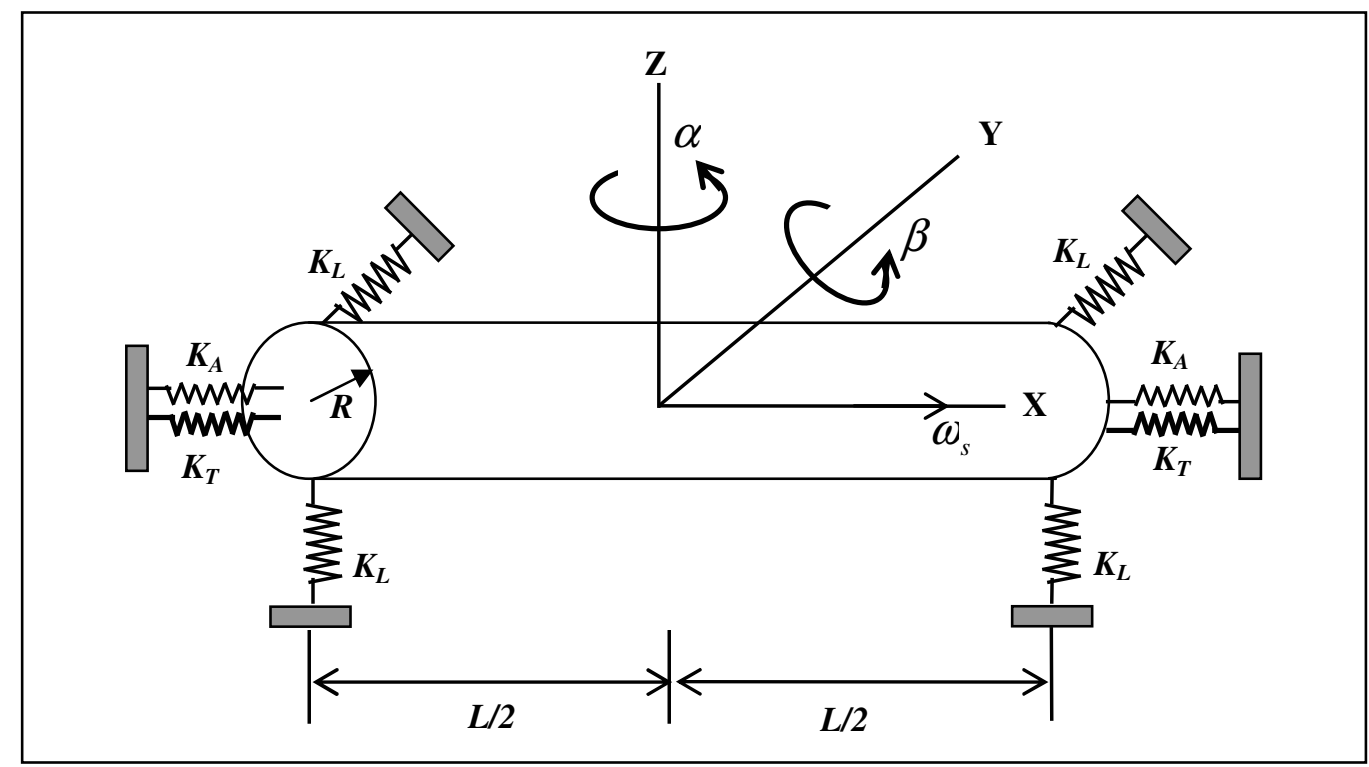

Fig. 6.4 Long Rigid-Rotor Model with Coordinate System

Parameter values utilized in the Fig.6.4 are shown in the Table 6.4 where $K_{L}$ linear bearing stiffness is attached to rotor, $K_{A}$ and $K_{T}$ indicate that axial and torsional spring, respectively. 
Table 6.4 Parameters of Long Rigid-Rotor Model

\begin{tabular}{ccccccc}
\hline \hline \multicolumn{7}{c}{ Long Rigid Rotor Model Parameters } \\
\hline$K_{L}$ & $K_{A}$ & $K_{T}$ & $L$ & $R$ & $\rho$ & $\omega_{s}$ \\
{$[\mathrm{~N} / \mathrm{m}]$} & {$[\mathrm{N} / \mathrm{m}]$} & {$[\mathrm{N} / \mathrm{m}]$} & {$[\mathrm{m}]$} & {$[\mathrm{m}]$} & {$\left[\mathrm{kg} / \mathrm{m}^{\wedge} 3\right]$} & {$[\mathrm{rpm}]$} \\
11290 & 56450 & 22580 & 0.762 & 0.05 & 7833 & 40000 \\
\hline \hline
\end{tabular}

Table 6.5 Comparison of Natural Frequencies between Analytical and FE Model

Case 1: Spin Speed is 0[rpm]

\begin{tabular}{|c|c|c|c|c|c|c|c|c|c|}
\hline \multicolumn{5}{|c|}{ Analytical Solution $[\mathrm{Hz}]$} & \multicolumn{5}{|c|}{ Finite Element Model [Hz] } \\
\hline$\omega_{1}$ & $\omega_{2}$ & $\omega_{3}$ & $\omega_{5}$ & $\omega_{6}$ & $\omega_{1}$ & $\omega_{2}$ & $\omega_{3}$ & $\omega_{5}$ & $\omega_{6}$ \\
\hline 135 & 135 & 232 & 191 & 214 & 135 & 135 & 223 & 191 & 213 \\
\hline \multicolumn{10}{|c|}{ Case 2: Spin Speed is $40000[\mathrm{rpm}]$} \\
\hline \multicolumn{5}{|c|}{ Analytical Solution [Hz] } & \multicolumn{5}{|c|}{ Finite Element Model [Hz] } \\
\hline$\omega_{1}$ & $\omega_{2}$ & $\omega_{3}$ & $\omega_{5}$ & $\omega_{6}$ & $\omega_{1}$ & $\omega_{2}$ & $\omega_{3}$ & $\omega_{5}$ & $\omega_{6}$ \\
\hline 135 & 135 & 241 & 191 & 214 & 135 & 135 & 232 & 191 & 213 \\
\hline
\end{tabular}




\subsection{Satellite Responses Including MB Suspension and Flexibility}

The reference motion is designed such that the satellite changes orientation 90[deg] about the Euler's Principal Axis (EPA) of rotation from the initial attitude $[s n]_{t i}$ to the final attitude $[s n]_{t f}$. The EPA is obtained as the eigenvector which corresponds to the eigenvalue +1 of the direction cosine matrix $[C]$

$$
[s n]_{t i}=\left[\begin{array}{ccc}
1 & 0 & 0 \\
0 & 1 & 0 \\
0 & 0 & 1
\end{array}\right] \quad[s n]_{t f}=\left[\begin{array}{rrr}
0.3952 & 0.0524 & 0.9170 \\
0.8037 & 0.4636 & -0.3729 \\
-0.4447 & 0.8844 & 0.1410
\end{array}\right] \quad[C]=[s n]_{t f}[s n]_{t i}^{T}
$$

then the EPA is $l=\left(\begin{array}{l}0.6286 \\ 0.6809 \\ 0.3756\end{array}\right)$

and the principal angle is $\Phi=\cos ^{-1}\left\{\frac{1}{2}\left(C_{11}+C_{22}+C_{33}-1\right)\right\}=90.00[\mathrm{deg}]$

Generally, the initial actual satellite orientation differs from the reference value. The initial attitude error in this present simulation is assumed to be $\left[\begin{array}{lll}-0.025 & 0.0375 & 0\end{array}\right]^{T}$ in terms of the Modified Rodriguez Parameter $(\delta \sigma)$, which corresponds to a 10.3 [deg] principal rotation angle deviation from the reference motion. The reference maneuver rotation is a $90.00[\mathrm{deg}]$ EPA change in $60[\mathrm{sec}]$ as shown in Fig.6.5. Fig.6.6 shows the satellite's motions with the tetrahedral array of four rigid shaft flywheels, 2 flexible appendages and the AMB suspension system for the case of a 10.3[deg] initial orientation error. The final rotational angle is 89.99 [deg] compared to the 90.00 [deg] 
target. The satellite's translational motion is negligible and the satellite's angular velocity and orientation errors diminish to zero after about 40 [sec] as shown in the Fig.6.7. As mentioned before, in the Chapter III (Flywheel motor control gain selection), the amplitude of satellite angular velocity error is designed to be half in the 4 [sec]. The angular velocity error shown in the Fig.6.7 is diminished almost half after 4 [sec]. The total torque applied to the satellite is shown in the Fig.6.8.
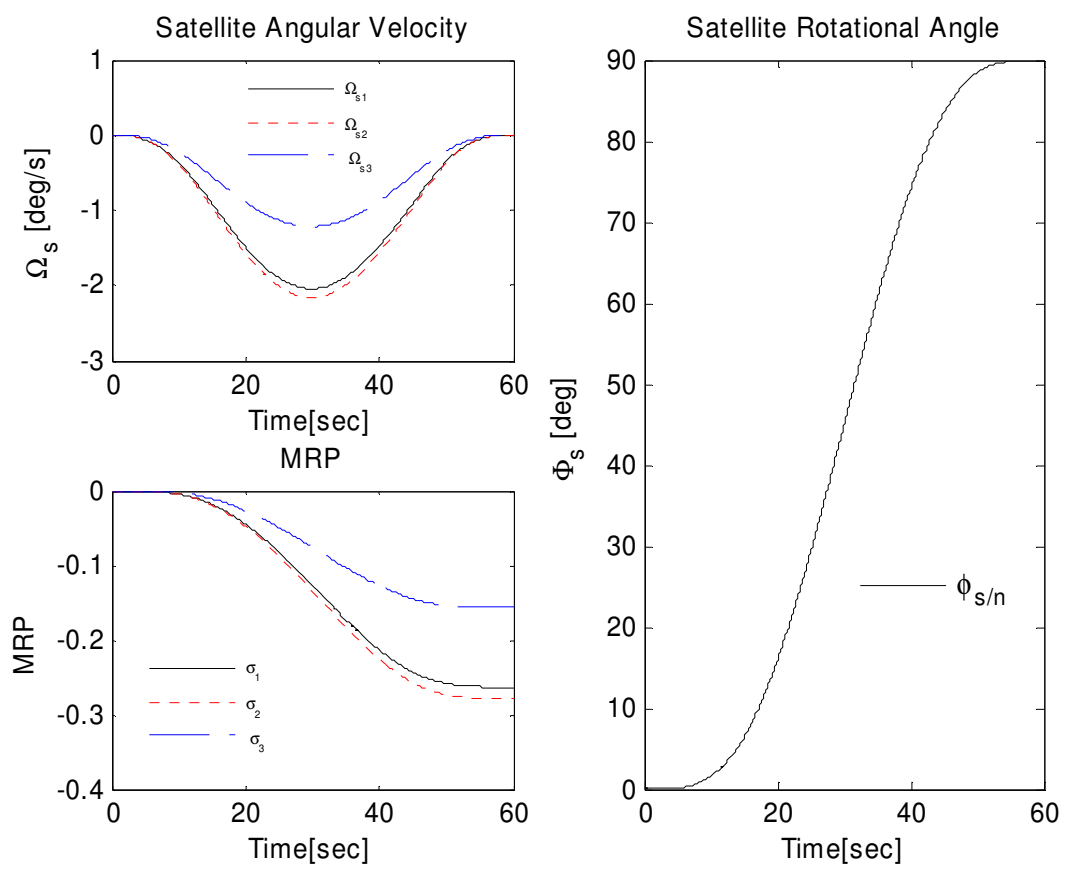

Fig. 6.5 Satellite Reference Motion 

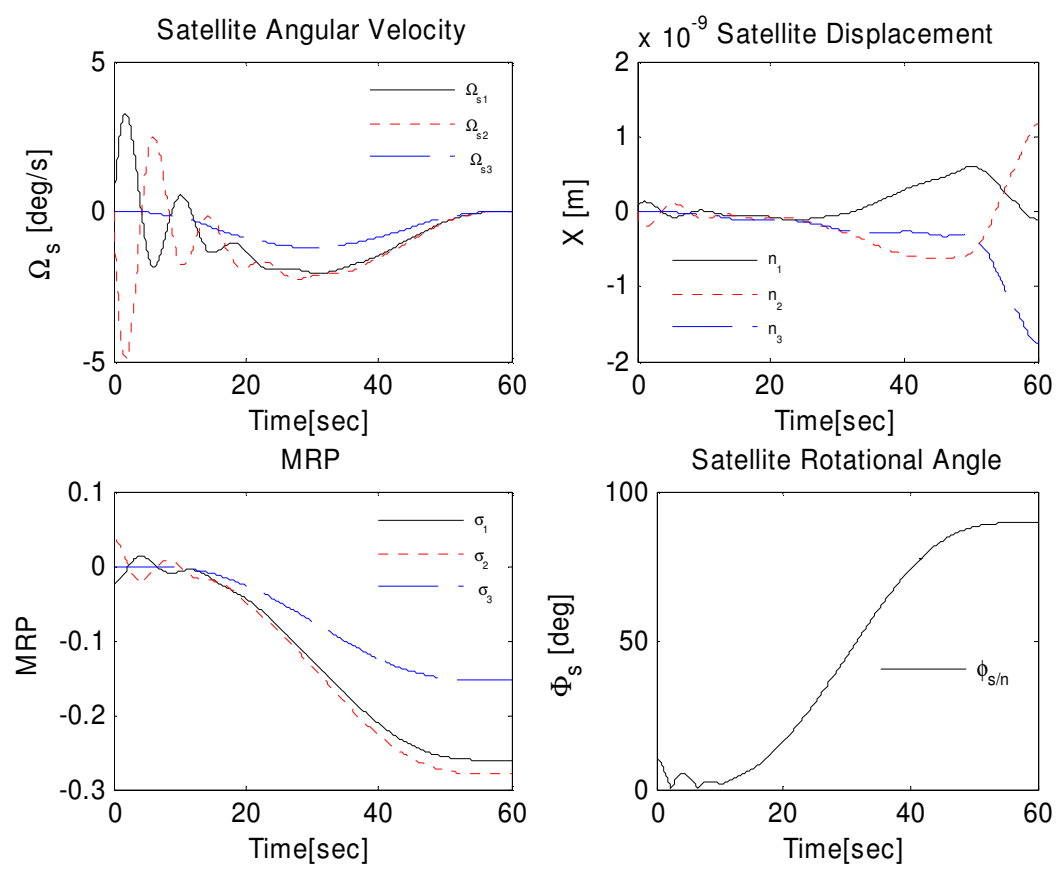

Fig. 6.6 Satellite Motions Including Flexibility and MB Suspension System
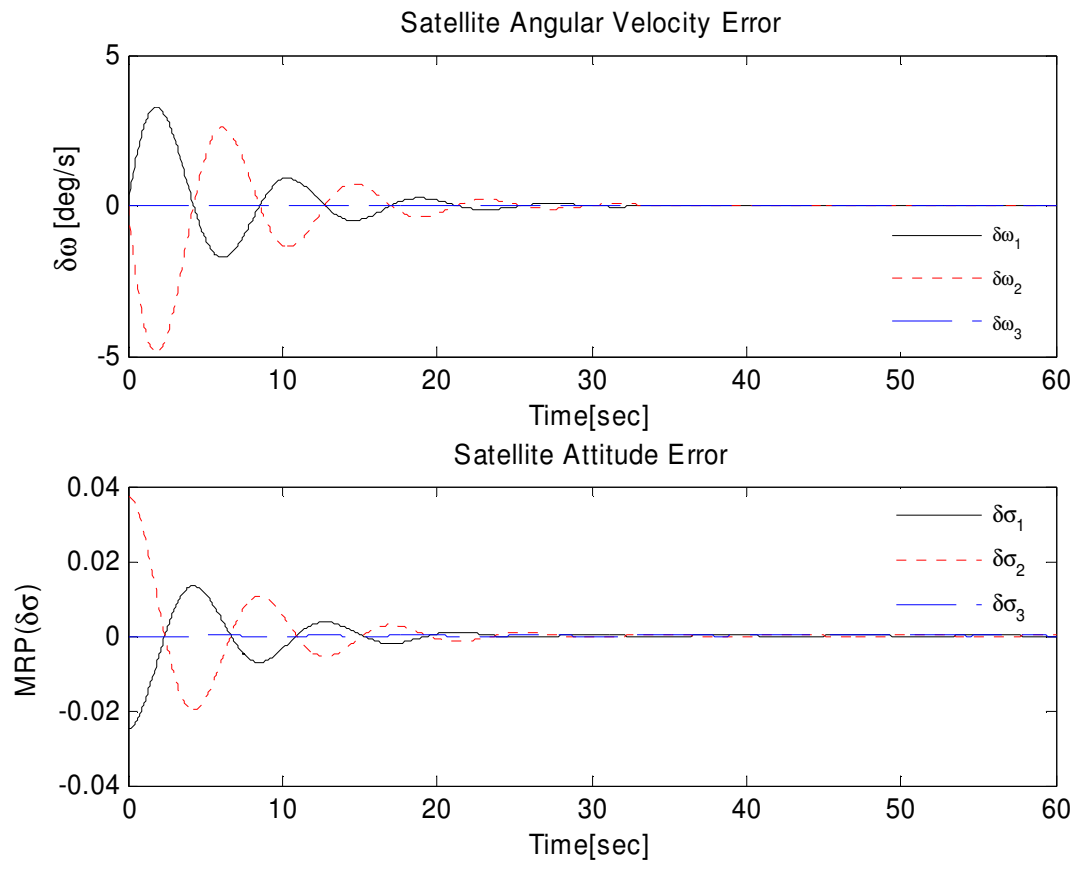

Fig. 6.7 Satellite Error Motions 

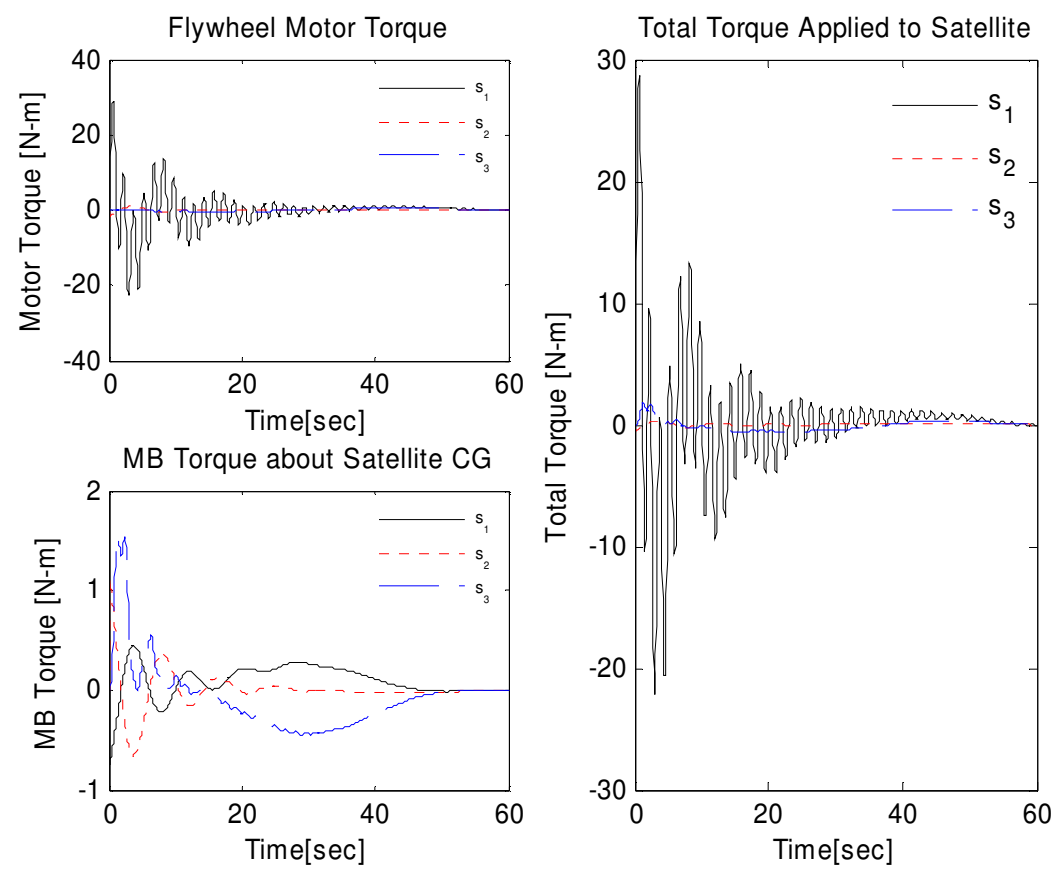

Fig. 6.8 Torques Applied to the Satellite

\subsection{Comparison of SISO and MIMO AMB Suspension Control}

This section compares the robustness of SISO and MIMO control for the case of a $I_{P} / I_{T}=1.25$ flywheel polar to transverse inertia ratio, and PD controller bandwidth of $1024[\mathrm{~Hz}]$ for both SISO and MIMO approaches. Saturation states were imposed on the actuator forces at a level of $444.8[N]=100[l b]$, on the voltage applied across the magnetic bearing coils at 80 [volt] and relative displacement of the flywheel is limited by nominal air gap which is defined in the section 4.4, $(c=0.020[i n c h]=5 e-4[\mathrm{~m}])$. All attempts to identify stable gains for the decentralized, PD, SISO controller failed, as documented in the Figs below. Control requirements to simultaneously reject the initial position error and imbalance disturbances, maintain the force and coil voltages in an unsaturated state and provide sufficient gain margin to overcome the controller phase 
lags could only be met by the MIMO controller despite many efforts to optimize the SISO controller. The physical reason for this result lies in the MIMO control's ability to cancel the gyroscopic torque that drives the forward and backward conical modes of each flywheel to extremely high or extremely low frequencies, respectively. Fig.6.9 shows how the relative displacements of the flywheels diverge at each module with SISO control. Fig.6.11 and 6.13 show that the corresponding AMB forces and coil voltages oscillate between their $(+)$ and $(-)$ saturation values. Fig.6.10, 6.12 and 6.14 show analogous plots for the MIMO control. Stable and unsaturated operations are maintained throughout the satellite model's simulated IPAC operation.
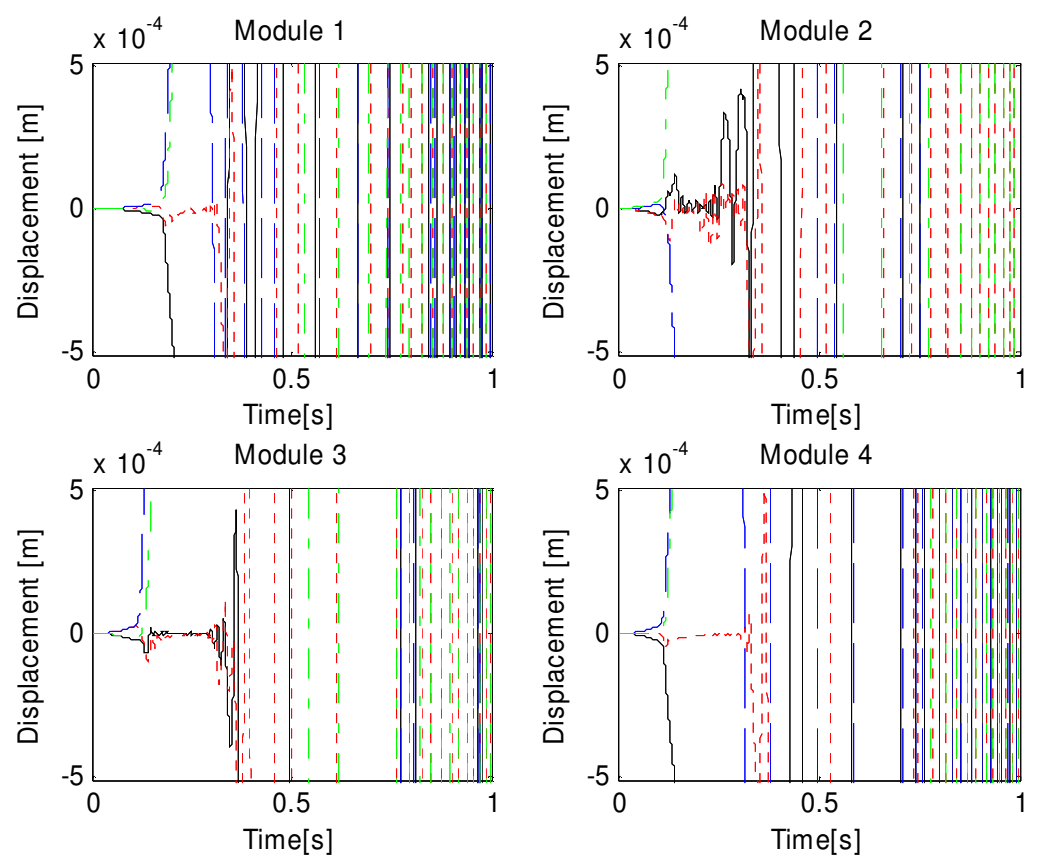

Fig. 6.9 Displacements of Flywheels at Sensor Position with SISO Control 

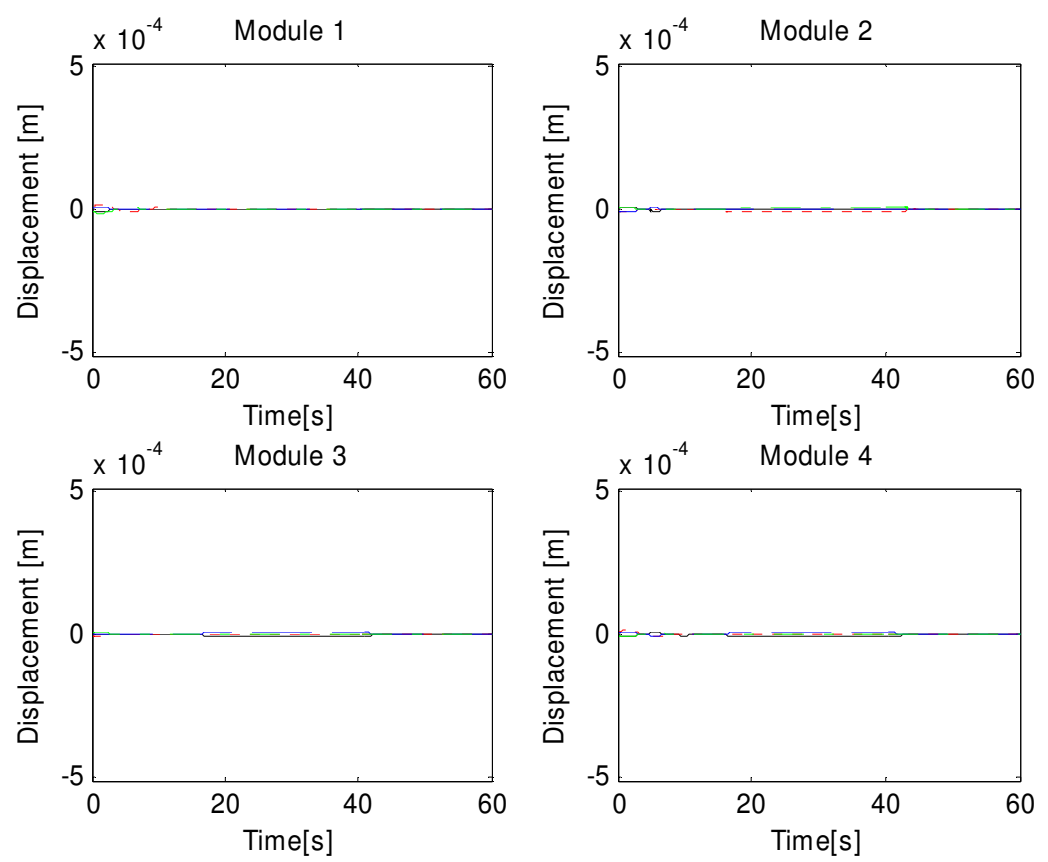

Fig. 6.10 Displacements of Flywheels at Sensor Position with MIMO Control
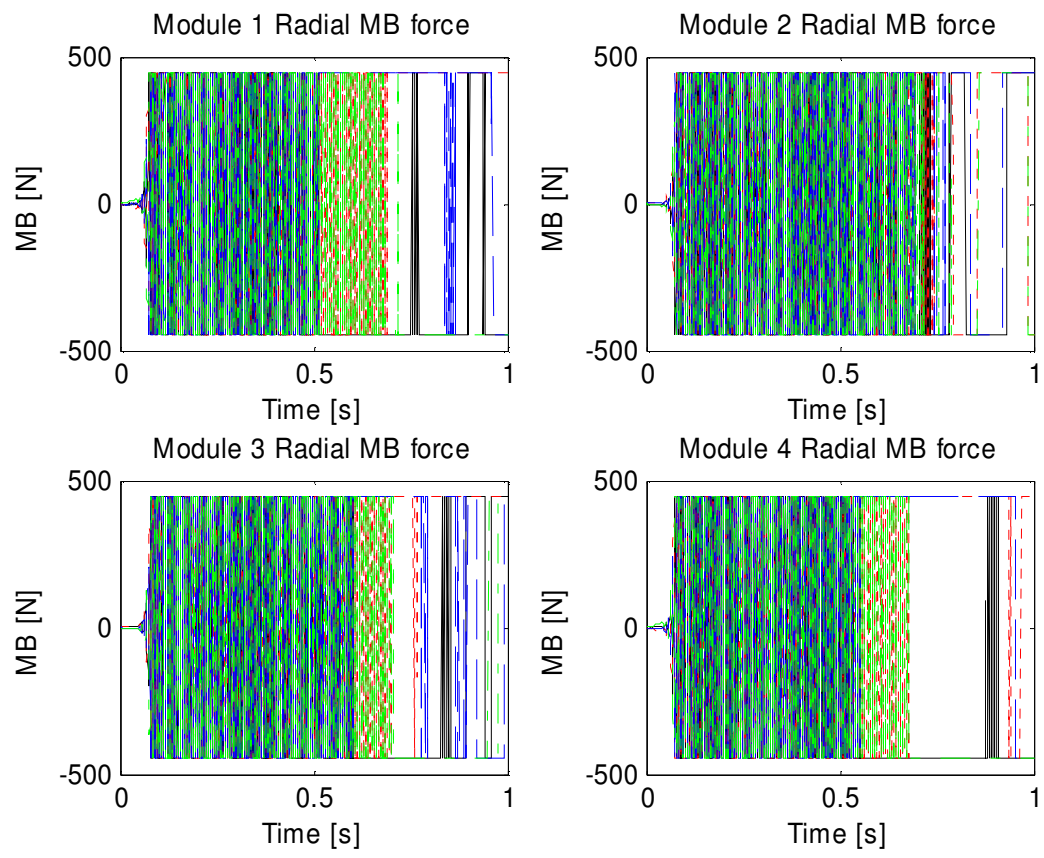

Fig. 6.11 MB Forces at Each Module (SISO Control) 

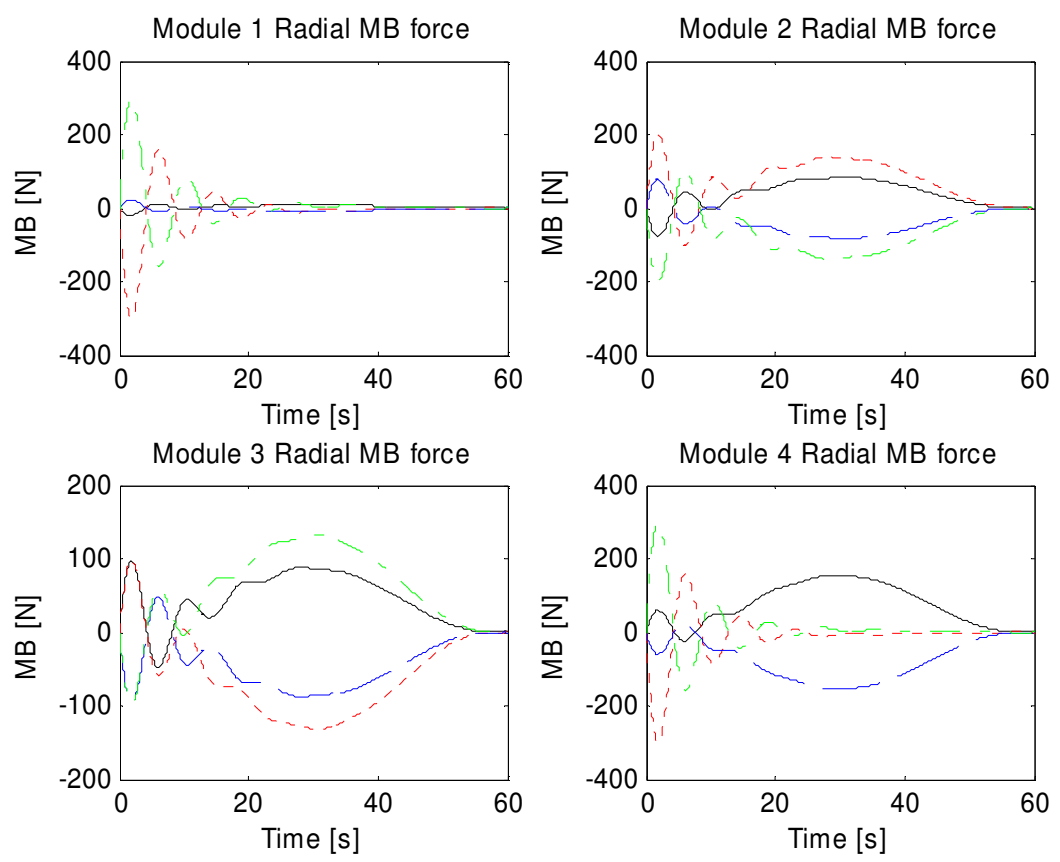

Fig. 6.12 MB Forces at Each Module (MIMO Control)
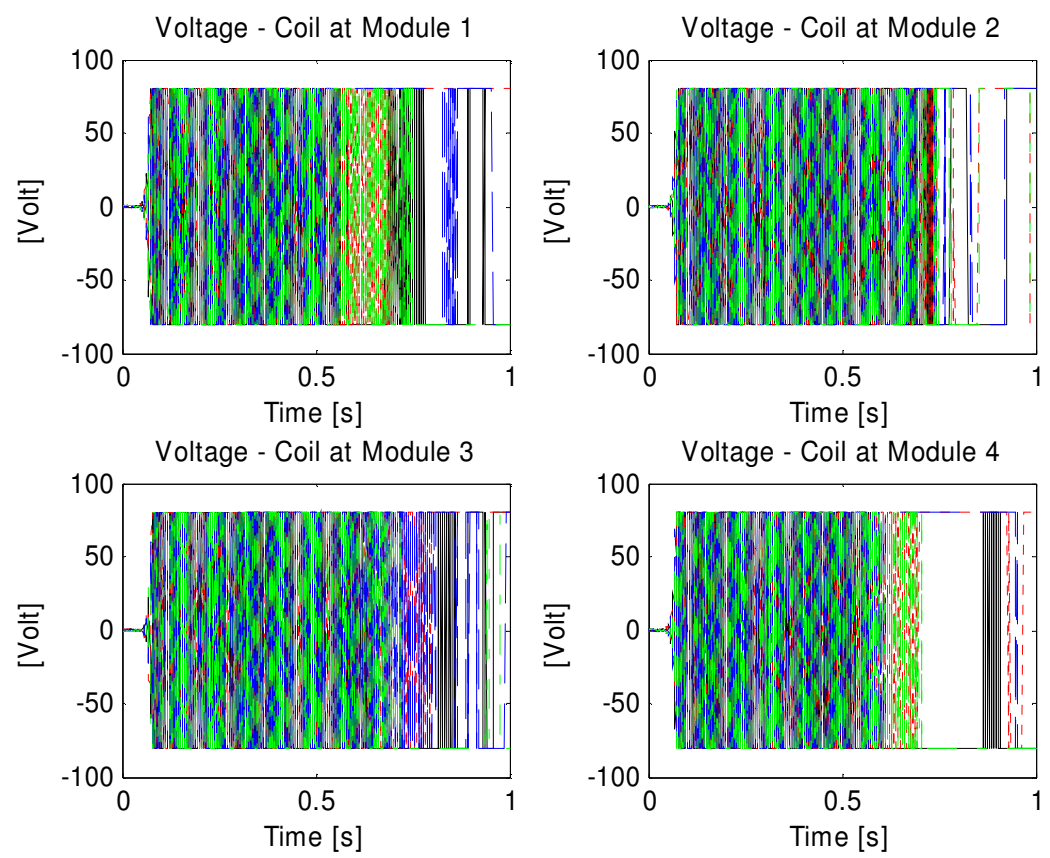

Fig. 6.13 Coil Voltages with SISO Control 

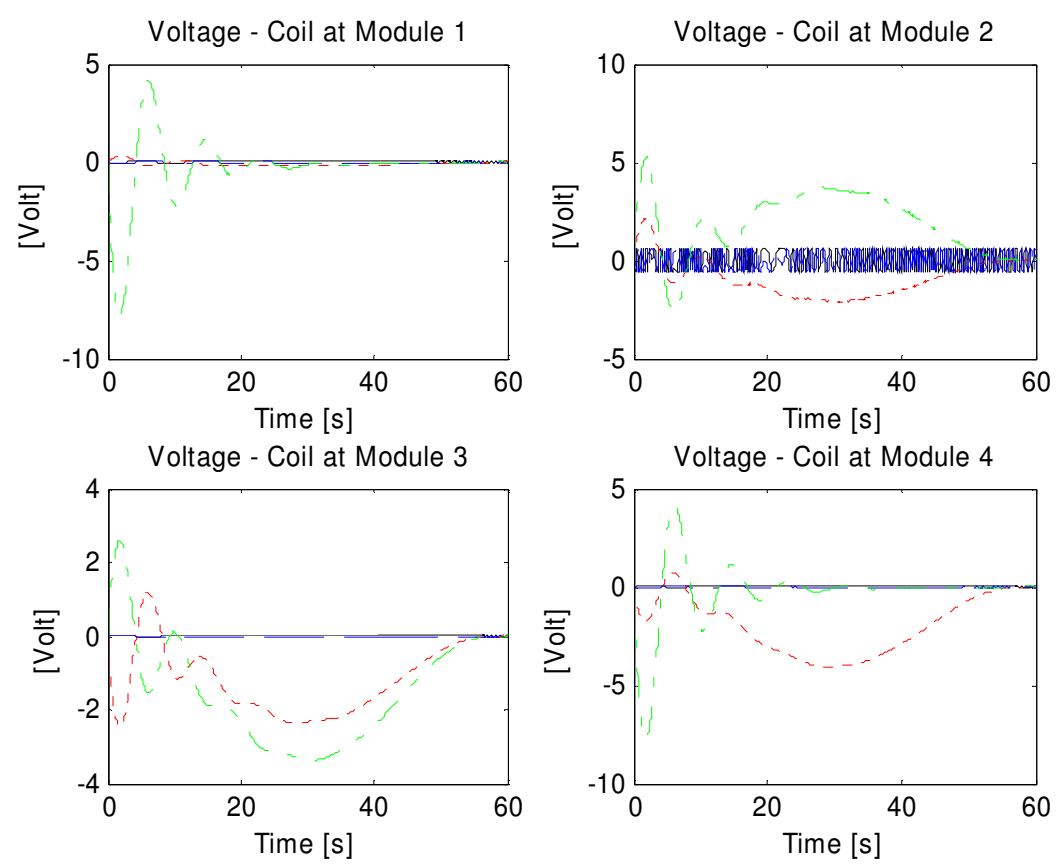

Fig. 6.14 Coil Voltages with MIMO Control

\subsection{VCM Effects on Flywheels and Flexible Appendages Motions}

Each appendage is modeled with 5 rigid disks connected by flexible Euler-Bernoulli

type beam elements. The number of disks is arbitrary and could be easily increased in the model. The proportional damping matrix is employed to stabilize satellite appendage motions. From modal analysis [30], the proportional damping ratio satisfies. $2 \xi \omega=\alpha+\beta \omega^{2}$. If $\alpha$ is selected to be zero, the damping ratio can be expressed as $\xi=\beta \omega / 2$. It is assumed that the appendage damping ratio increases linearly with frequency, with approximately $5[\%]$ damping at $2[\mathrm{~Hz}]$ for the sake of illustration. The last 2 Figs in this section 6.6, the different results are presented between 5[\%] and 2[\%] damping at the same satellite appendage vibration frequency. The vibration control mass (VCM) to attenuate flexible appendage is selected as $1.35[\mathrm{Kg}]$ on both ends. The 
following Figs are consist of flywheel power charging and delivery case.

\subsubsection{VCM Effects on Flywheel Power Charging Case}

Figures $6.15,6.16,6.17$ and 6.18 show the flywheel motions and attitude control power charging torques with and without the VCM. These figures confirm that the "without VCM" oscillations are significantly higher. Power charging responses for the "with and without" VCM cases are shown in Fig.6.19 and 6.20. For illustration, the IPAC operation consists of charging (power transfer) the flywheels at a rate of 500 [watt] for 30 [sec], while the satellite is simultaneously rotated by 90 degrees over a 60 second period. Fig.6.21 and 6.22 show the magnified power charging responses of Fig. 6.19(e) and 6.20(e) revealing that the power fluctuation is significantly reduced by the VCM.
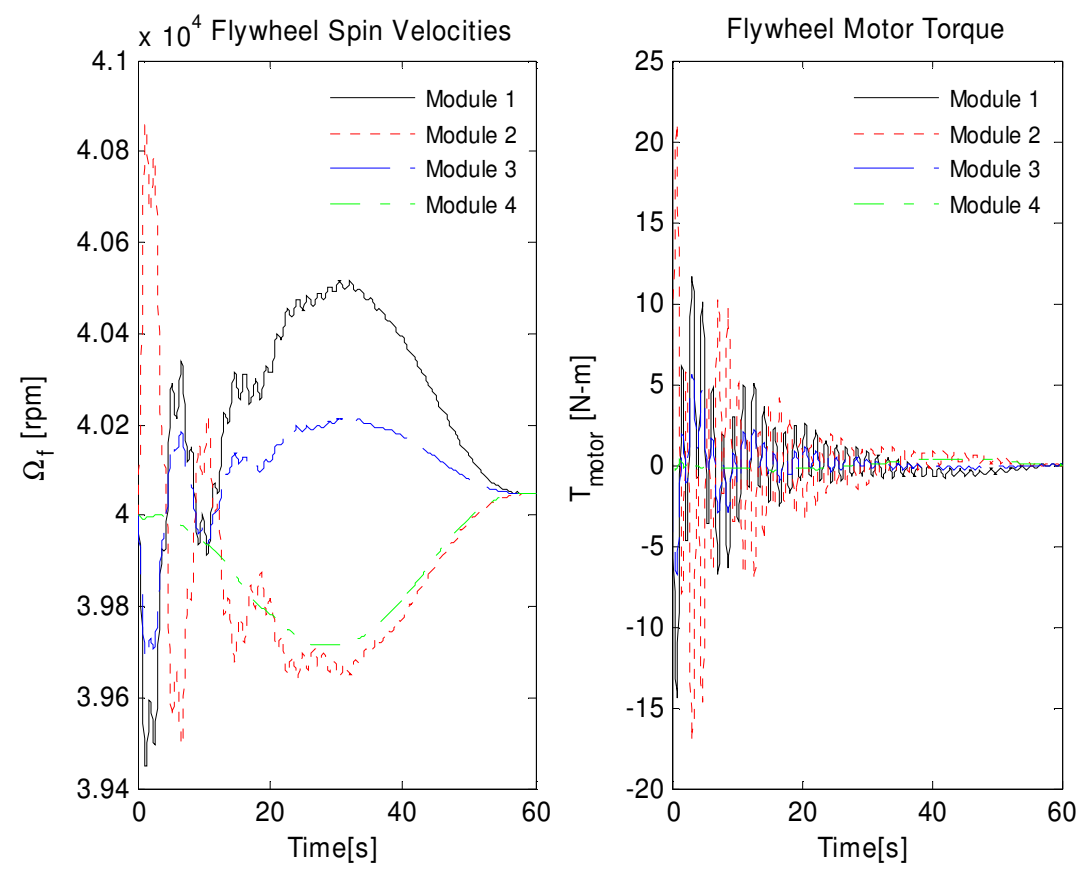

Fig. 6.15 Flywheel Motions without VCM for Power Charging Case 

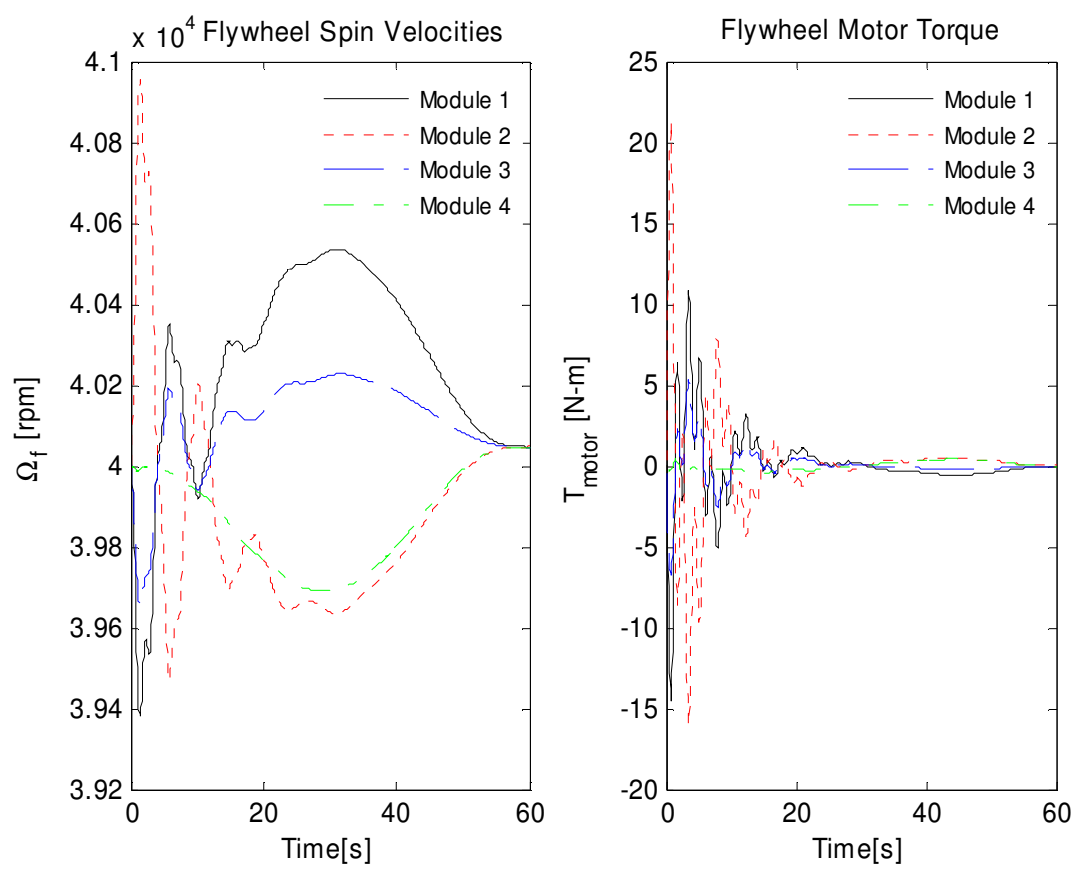

Fig. 6.16 Flywheel Motions with VCM (1.35[kg]) for Power Charging Case
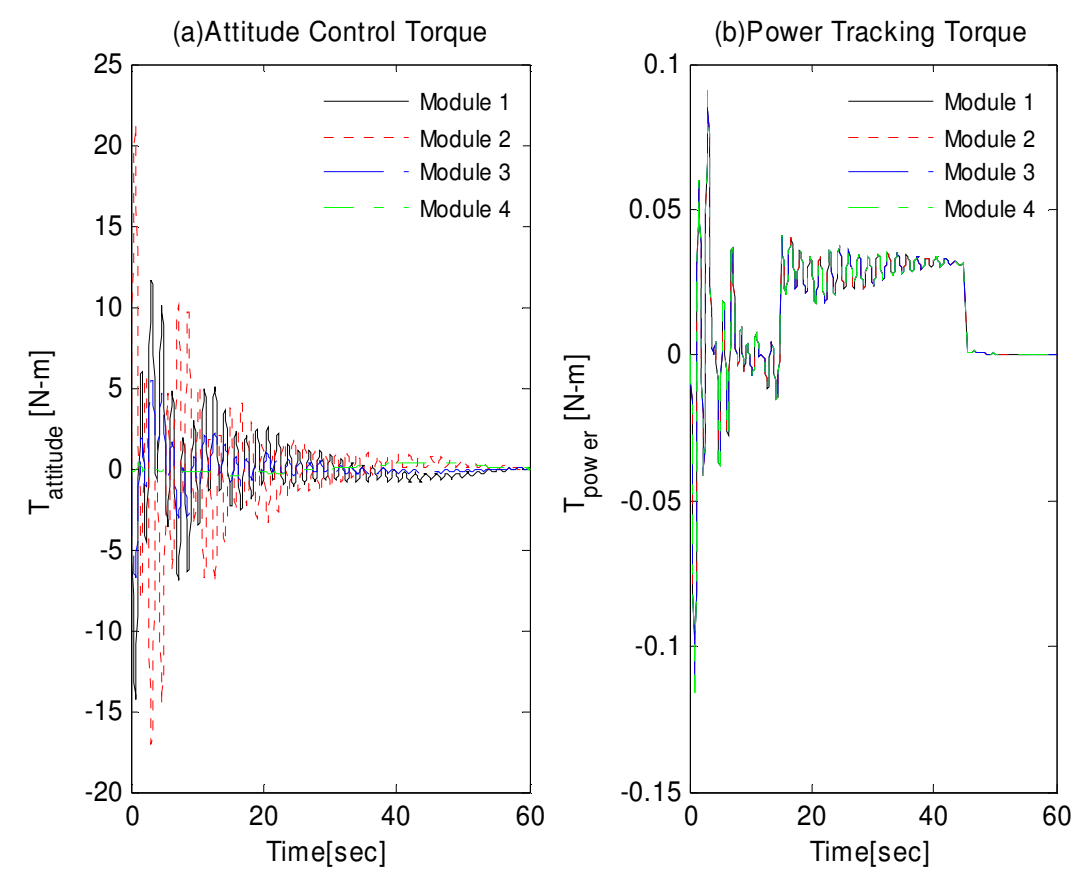

Fig. 6.17 Attitude Control Torque and Power Charging Torque without VCM 

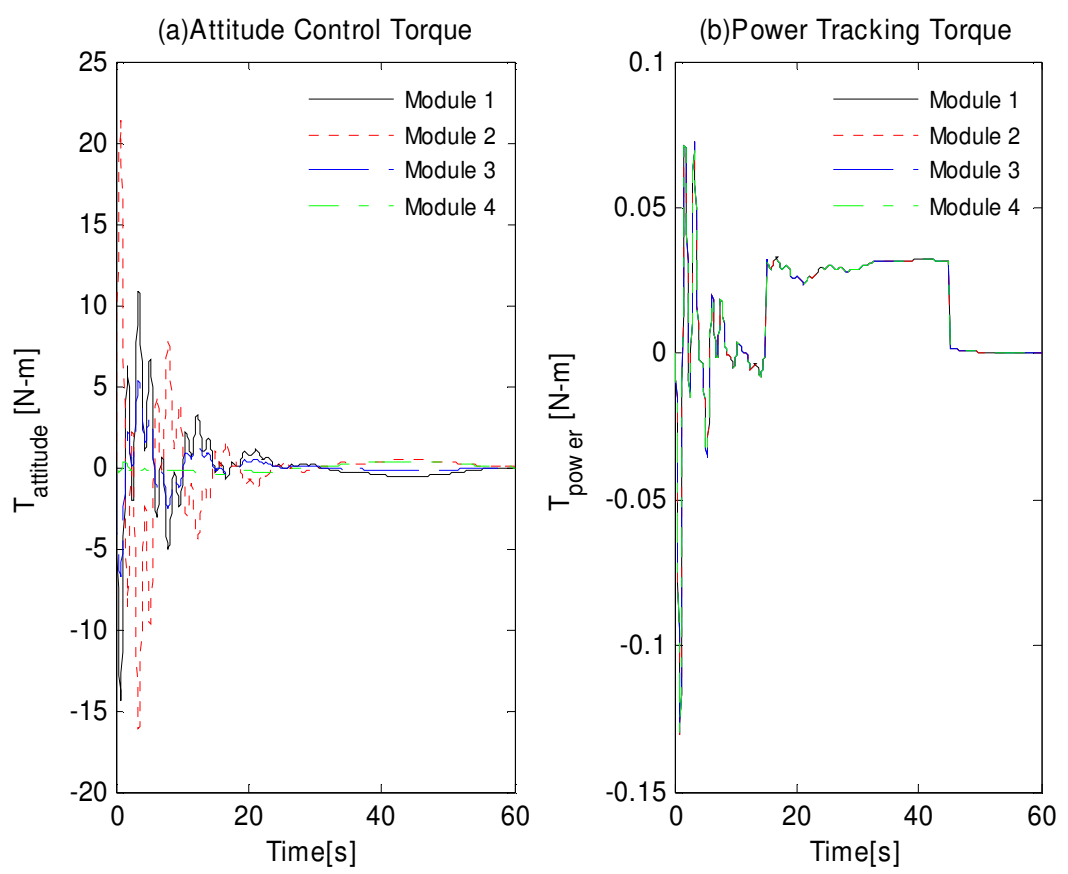

Fig. 6.18 Attitude Control Torque and Power Charging Torque with VCM
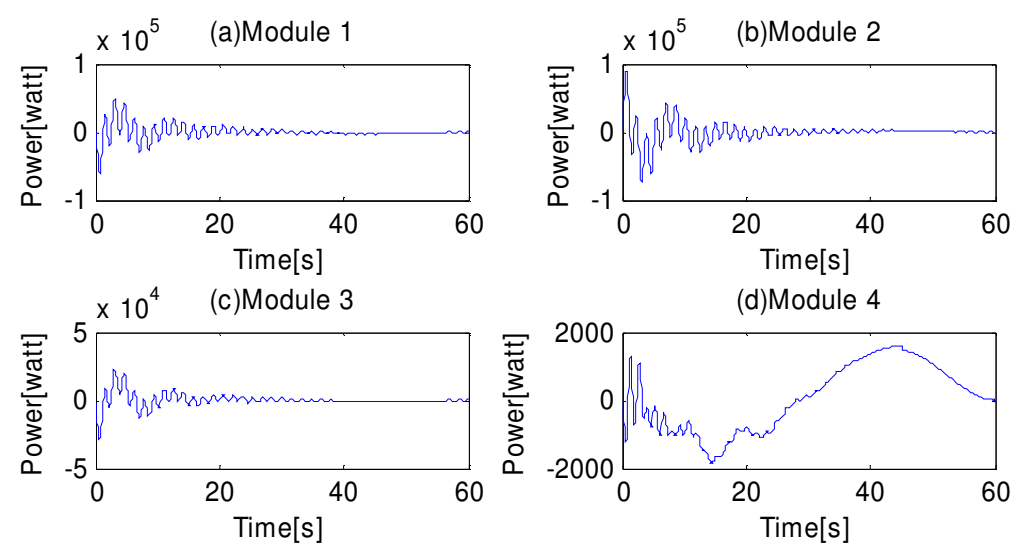

(e)Total Power

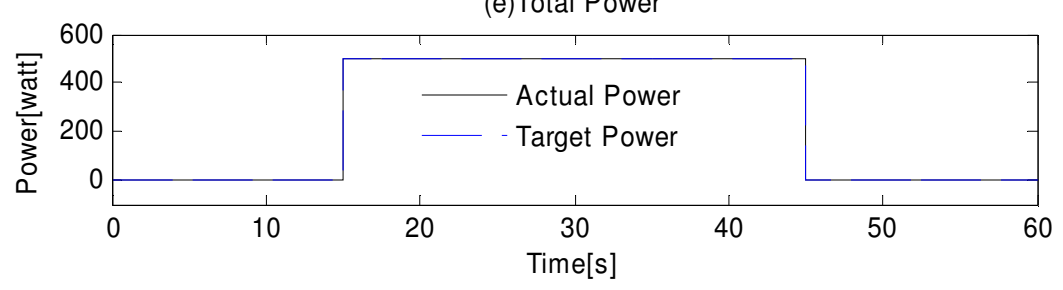

Fig. 6.19 Power Charging Response without VCM 

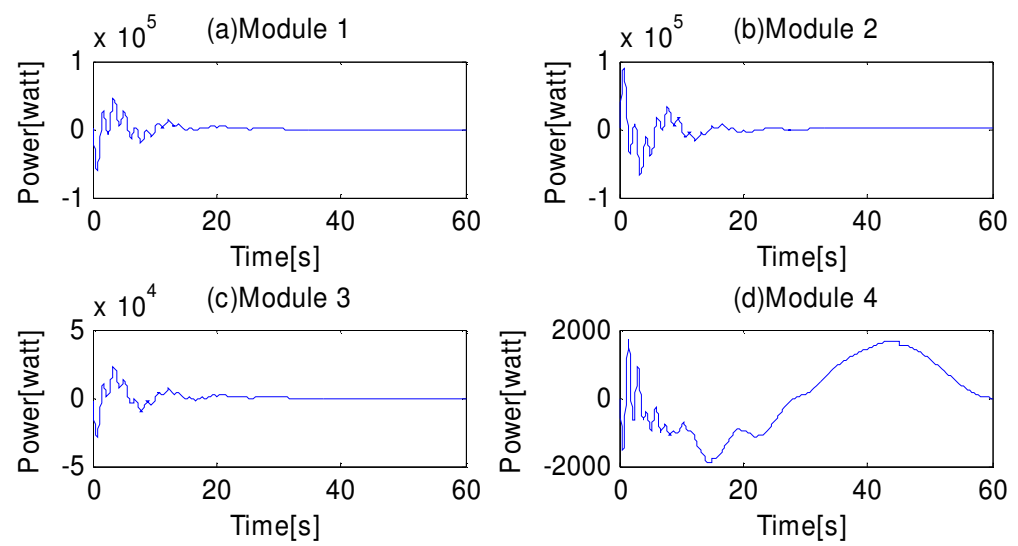

(e)Total Power

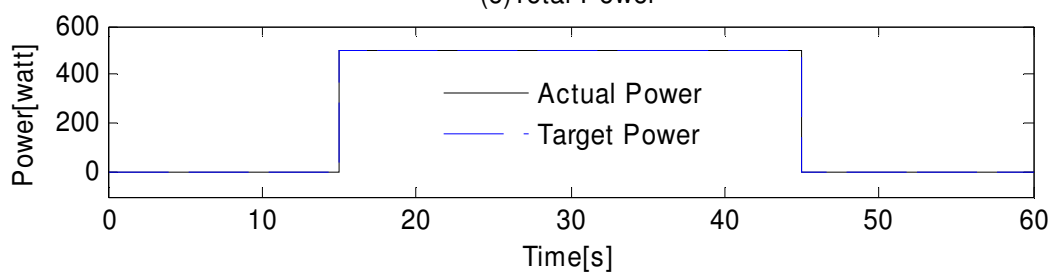

Fig. 6.20 Power Charging Response with VCM (1.35[kg])
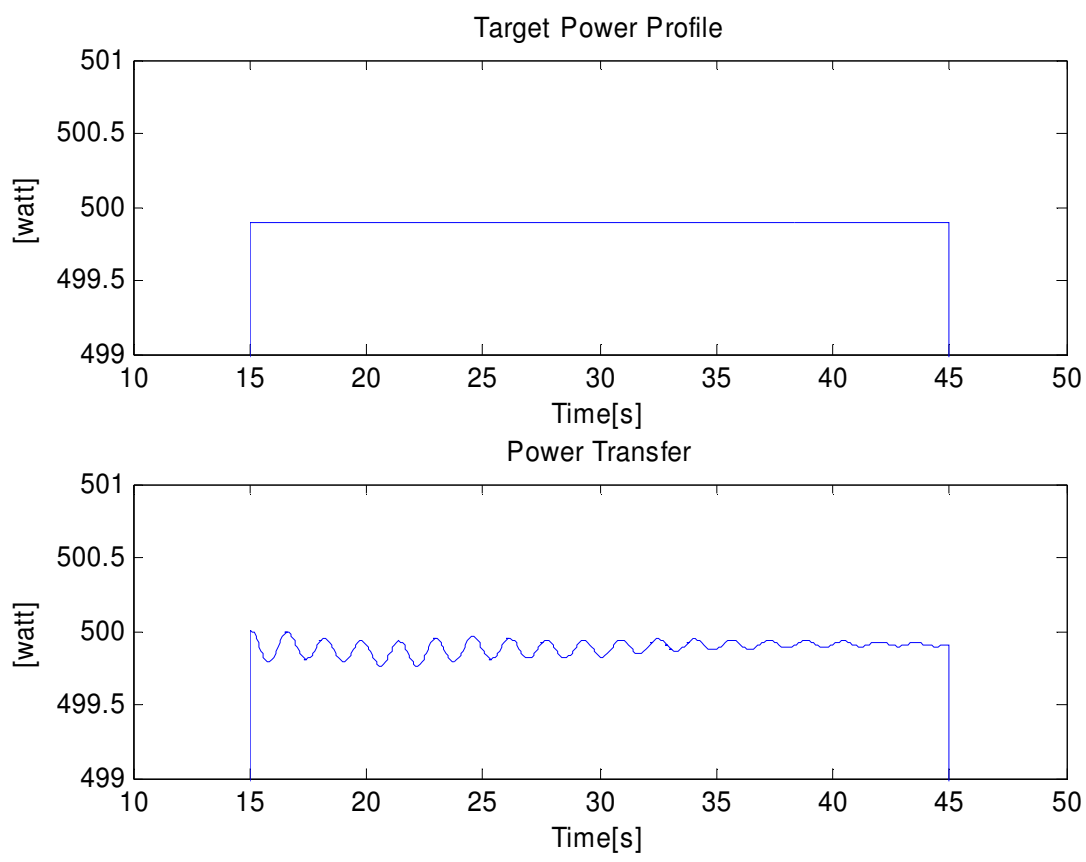

Fig. 6.21 Magnified Power Transfer (Charging) without VCM 

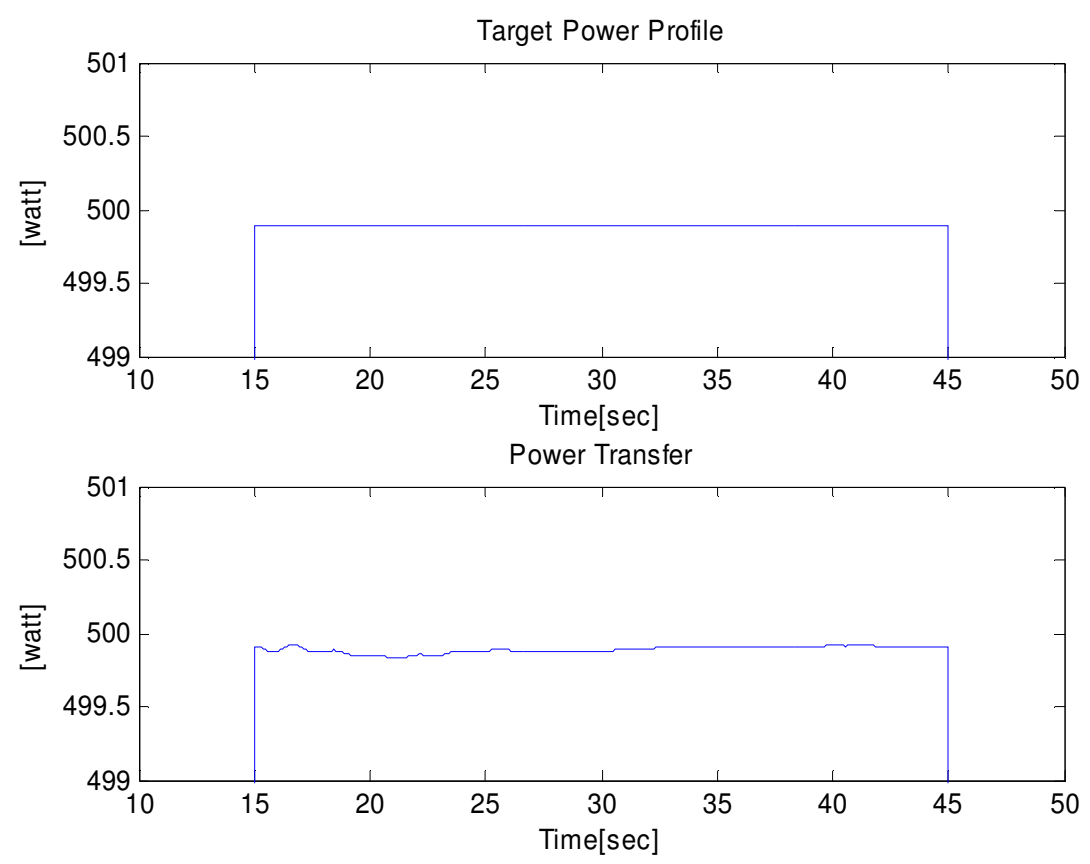

Fig. 6.22 Magnified Power Transfer (Charging) with VCM (1.35[kg])

\subsubsection{VCM Effects on Flywheel Power Delivery Case}

The Flywheel spin speed and flywheel motor torque including attitude and power delivery torques are plotted in the Fig. 6.23 and 6.24 with and without VCM. As same as power charging case, the oscillations of flywheel speed and motor torque are significantly attenuated compared these two Figs. As mentioned before, the flywheel motor torque consists of satellite attitude control torque and power transfer torque, Fig. 6.25 and 6.26 show that these two torques with and without VCM of $1.35[\mathrm{Kg}]$. Attitude control torque derived from range space and power delivery torque from null space performed that $90[\mathrm{deg}]$ rotation about principle axis over $60[\mathrm{sec}]$ and $500[\mathrm{watt}]$ power discharging for $30[\mathrm{sec}]$ successfully. Each flywheel module power delivery rate is shown in the Fig. 6.27 and 6.28 in the case of with and without VCM. Total power 
transfer, 500[watt] for 30[sec], is sum of each flywheel module shown (a), (b), (c) and (d) in these Figs. It can be easily recognized that the power fluctuation reduction compared between magnified total power transfer in the Fig. 6.29 and 6.30.
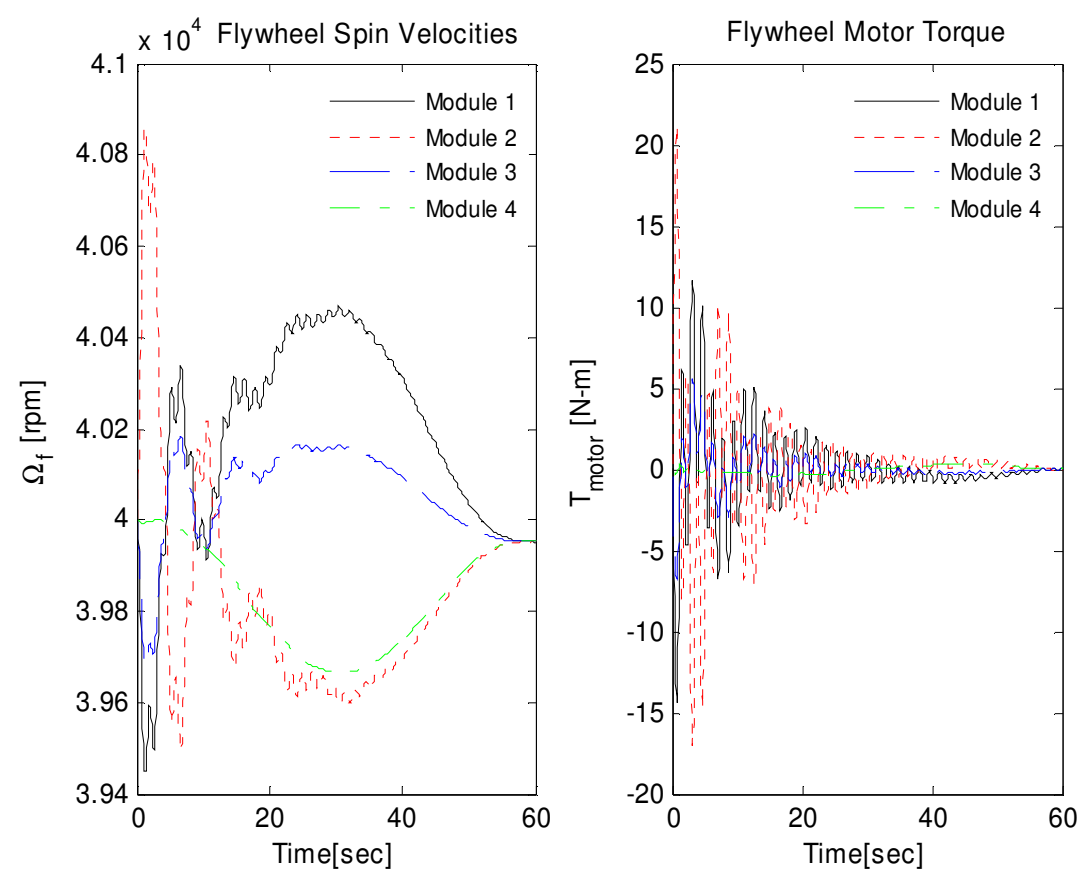

Fig. 6.23 Flywheel Motions without VCM for Power Delivery Case 

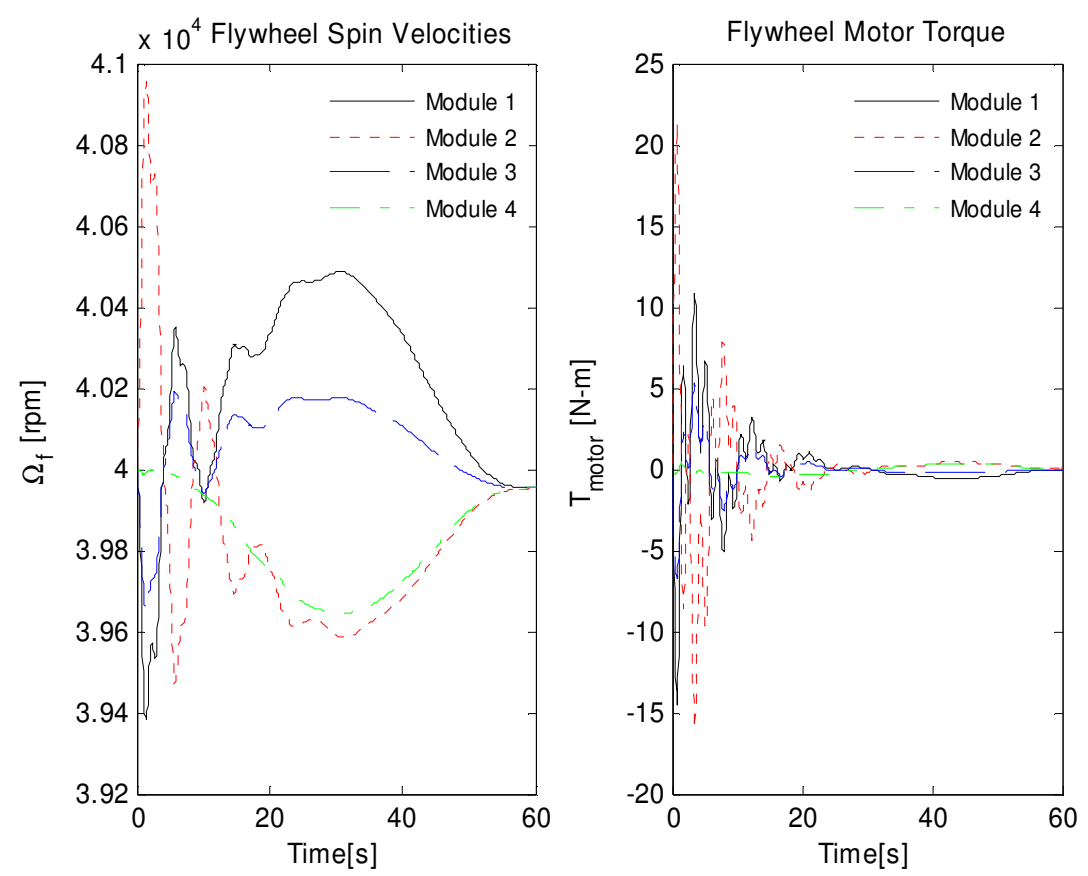

Fig. 6.24 Flywheel Motors with VCM (1.35[kg]) for Power Delivery Case
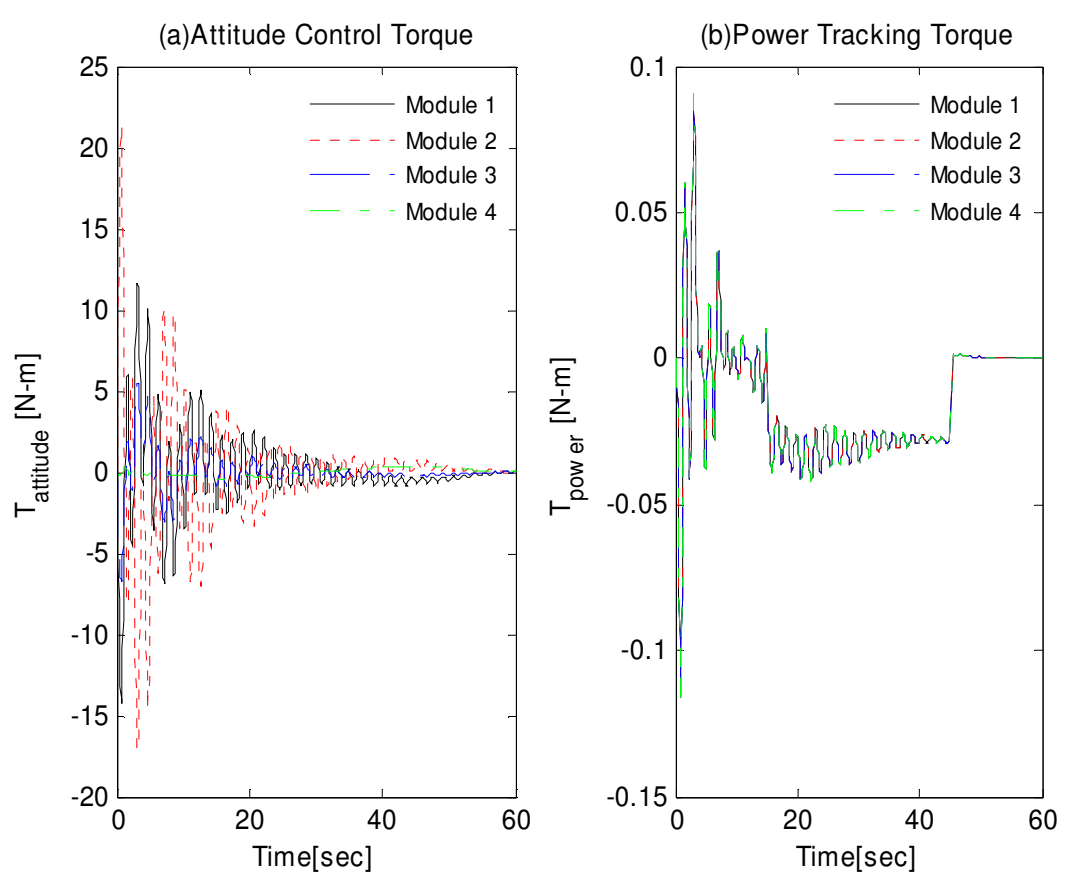

Fig. 6.25 Attitude Control Torque and Power Delivery Torque without VCM 

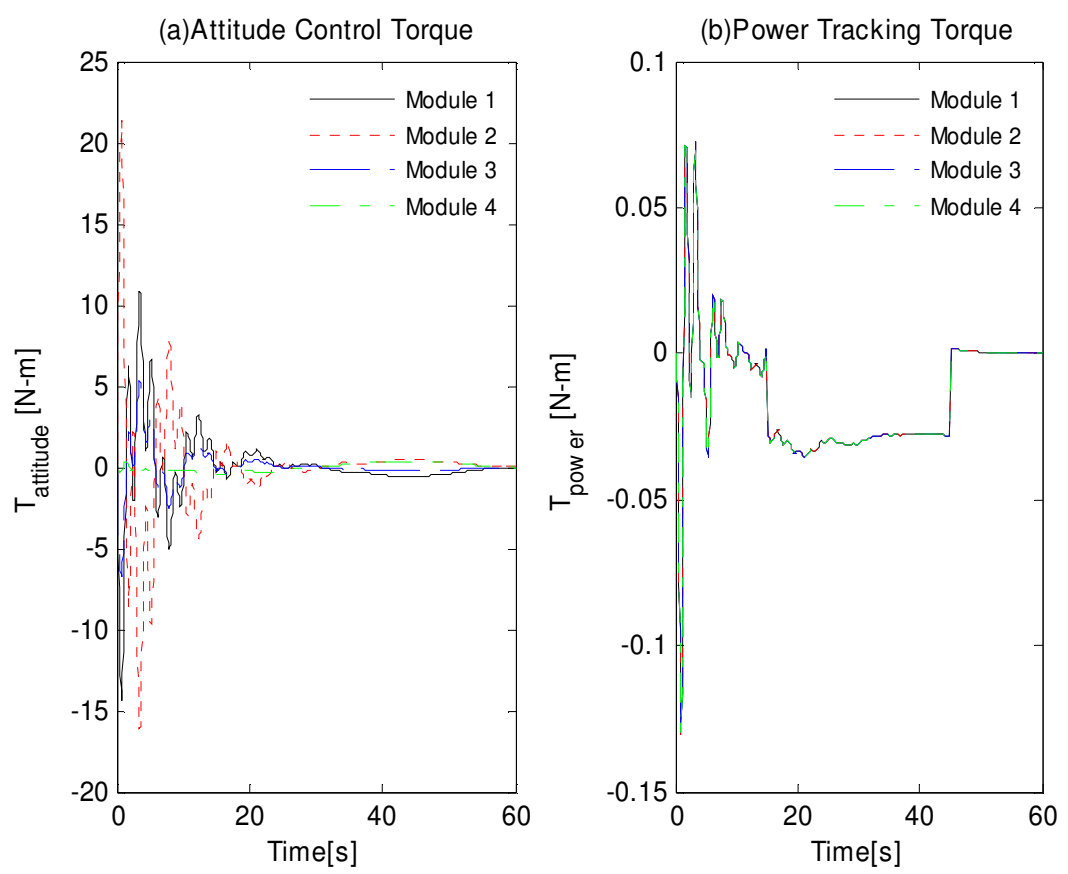

Fig. 6.26 Attitude Control Torque and Power Delivery Torque with VCM
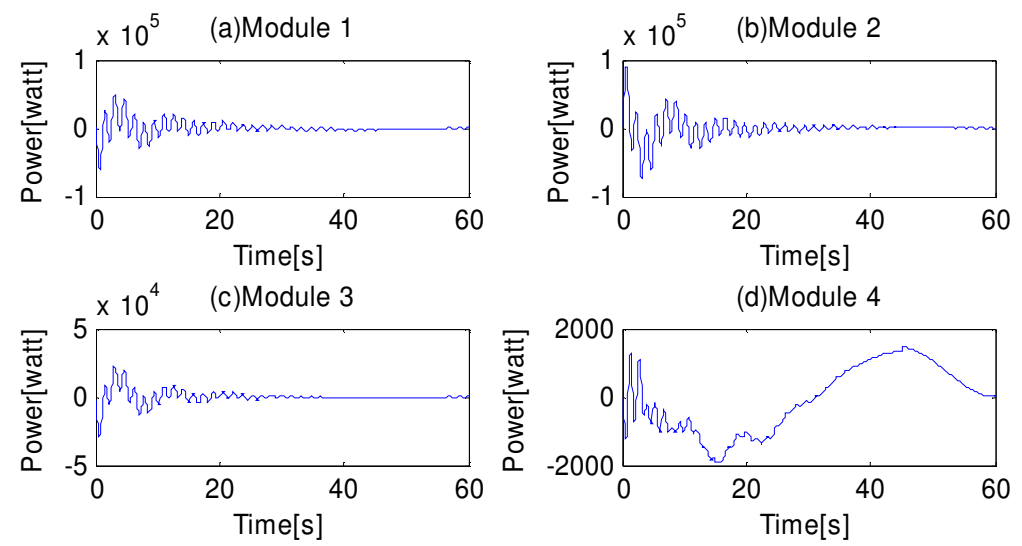

(e)Total Power

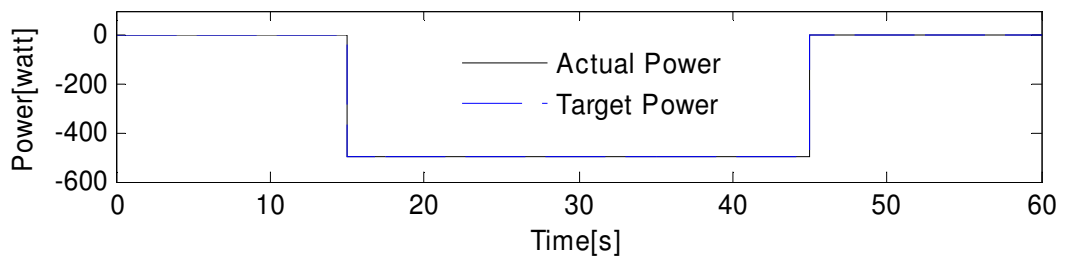

Fig. 6.27 Power Delivery Response without VCM 

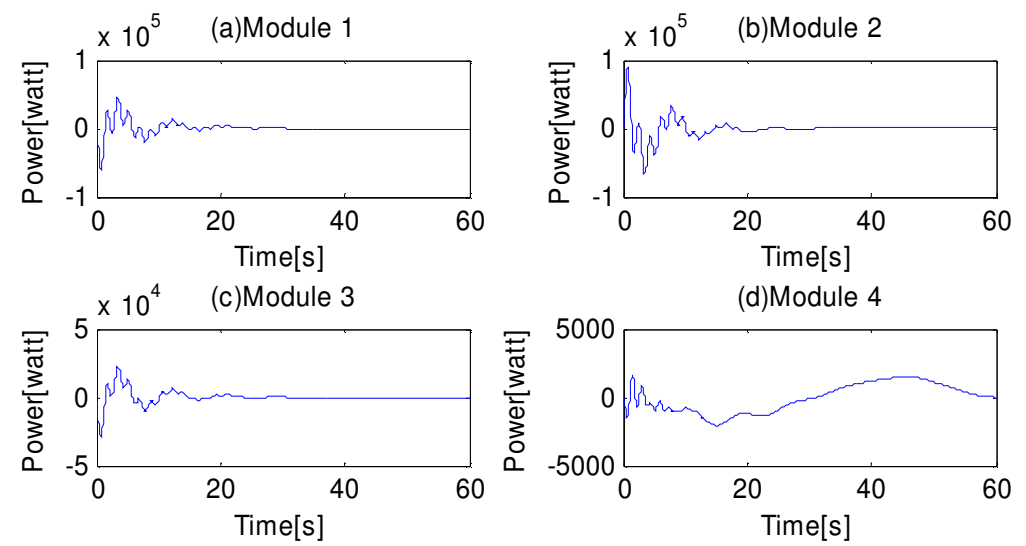

(e)Total Power

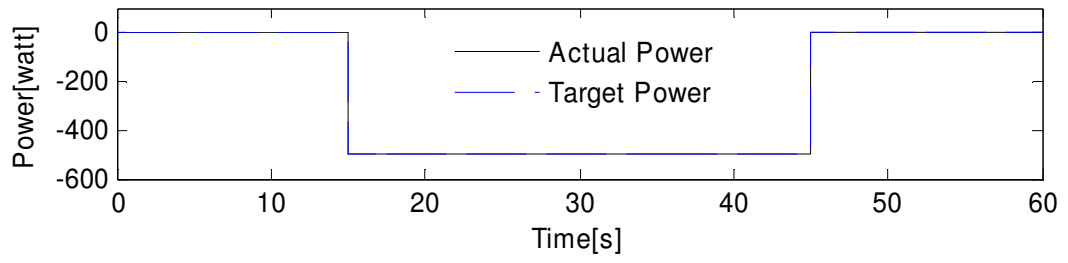

Fig. 6.28 Power Delivery Response with VCM $(1.35[\mathrm{~kg}])$

(a)Target Power profile

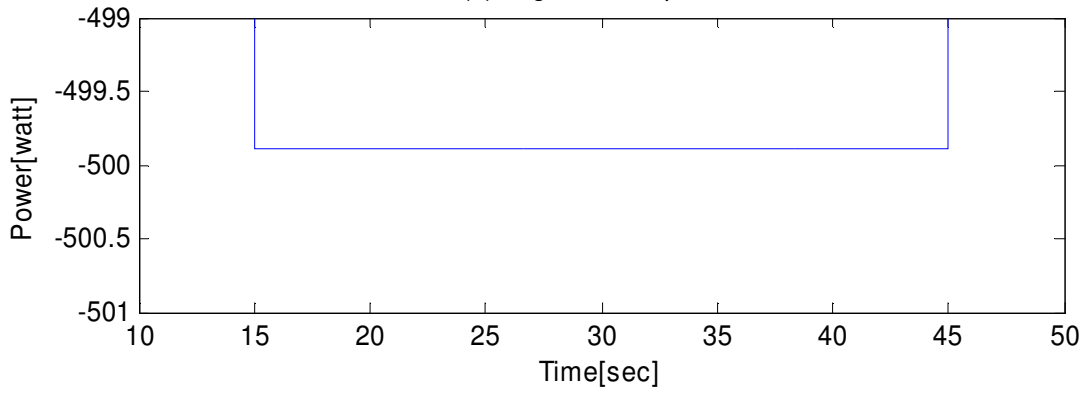

(b)Power Transfer

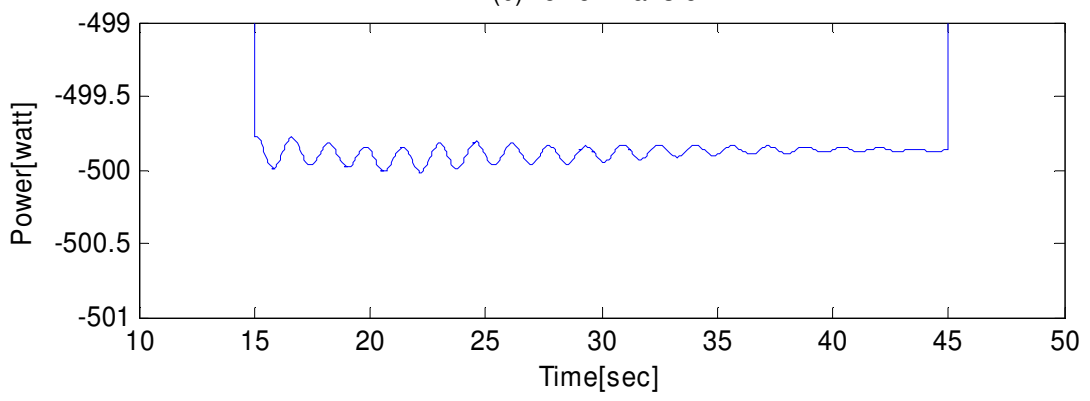

Fig. 6.29 Magnified Power Transfer (Delivery) without VCM 

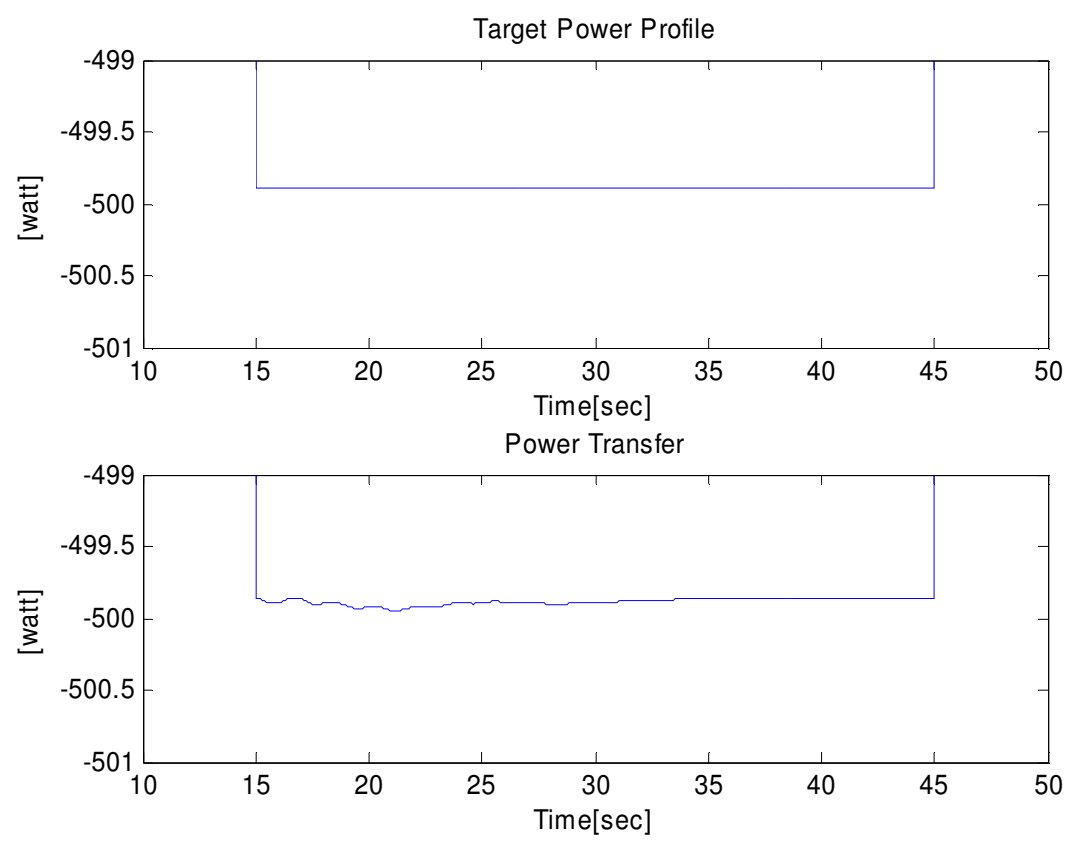

Fig. 6.30 Magnified Power Transfer (Delivery) with VCM (1.35[kg])

\subsubsection{VCM Effects on Flexible Appendages Motion}

Fig.6.31 and 6.32 show the translational motion of the appendage disks relative to the appendage reference line. The disk vibration amplitude increases as one moves along the appendage away from the satellite since the first disk is attached to the satellite with very stiff linear and torsional springs, so its amplitude is very small. Fig.6.32 demonstrates the ability of the VCM to reduce appendage vibration. Fig.6.33 and 6.34 show the maximum power ripple and the relative stroke (displacement difference between the VCM and disk 5) for varying VCM mass with $2 \%$ and 5\% damping ratio at $2[\mathrm{~Hz}$ ], respectively. The maximum power ripple is 0.23 [watt] for the case of no VCM, however, this ripple is reduced by about $70 \%$ with a $(1.35[\mathrm{~kg}]) \mathrm{VCM}$ at $5 \%$ damping ratio case. 

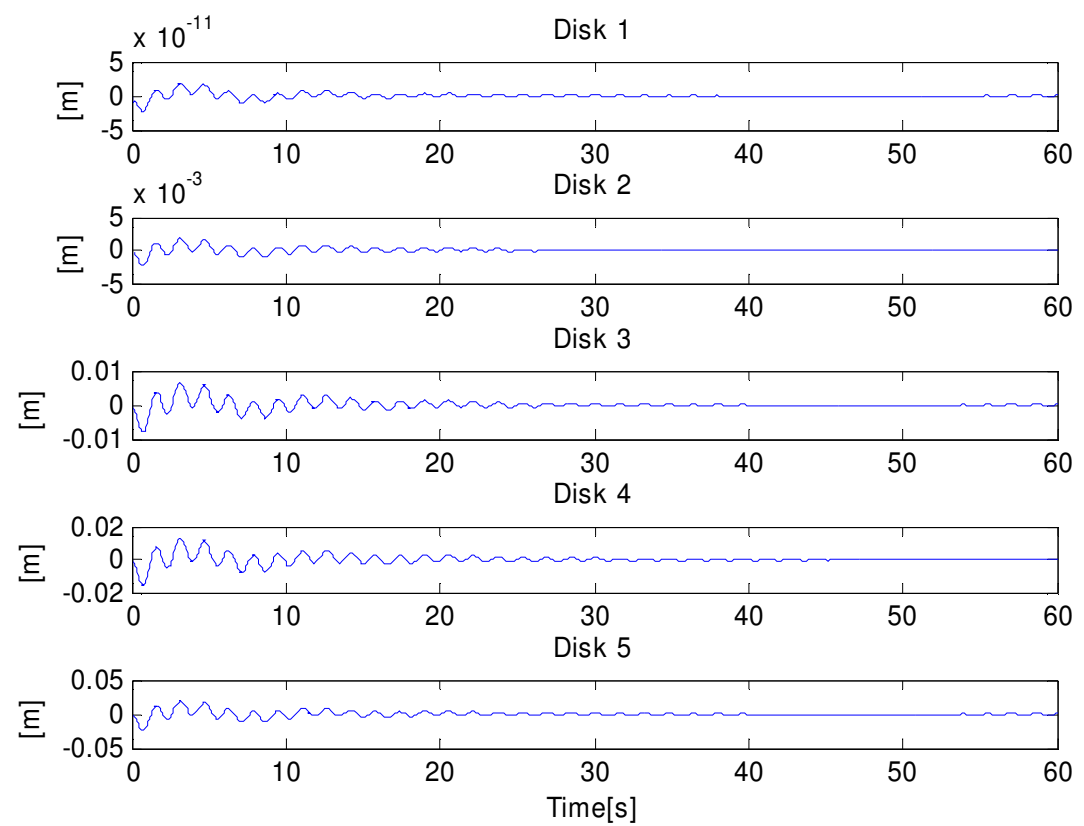

Fig. 6.31 Vibration along Satellite Appendage during IPAC without VCM
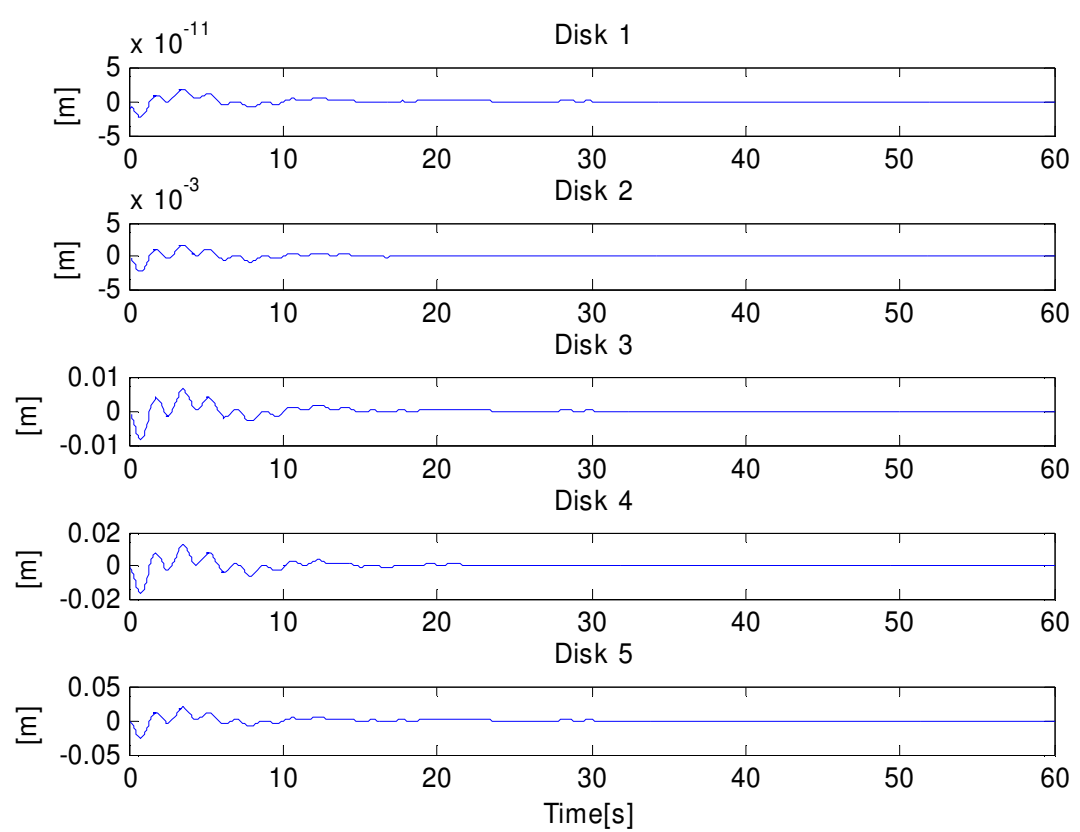

Fig. 6.32 Vibration along Satellite Appendage during IPAC with VCM (1.35[kg]) 


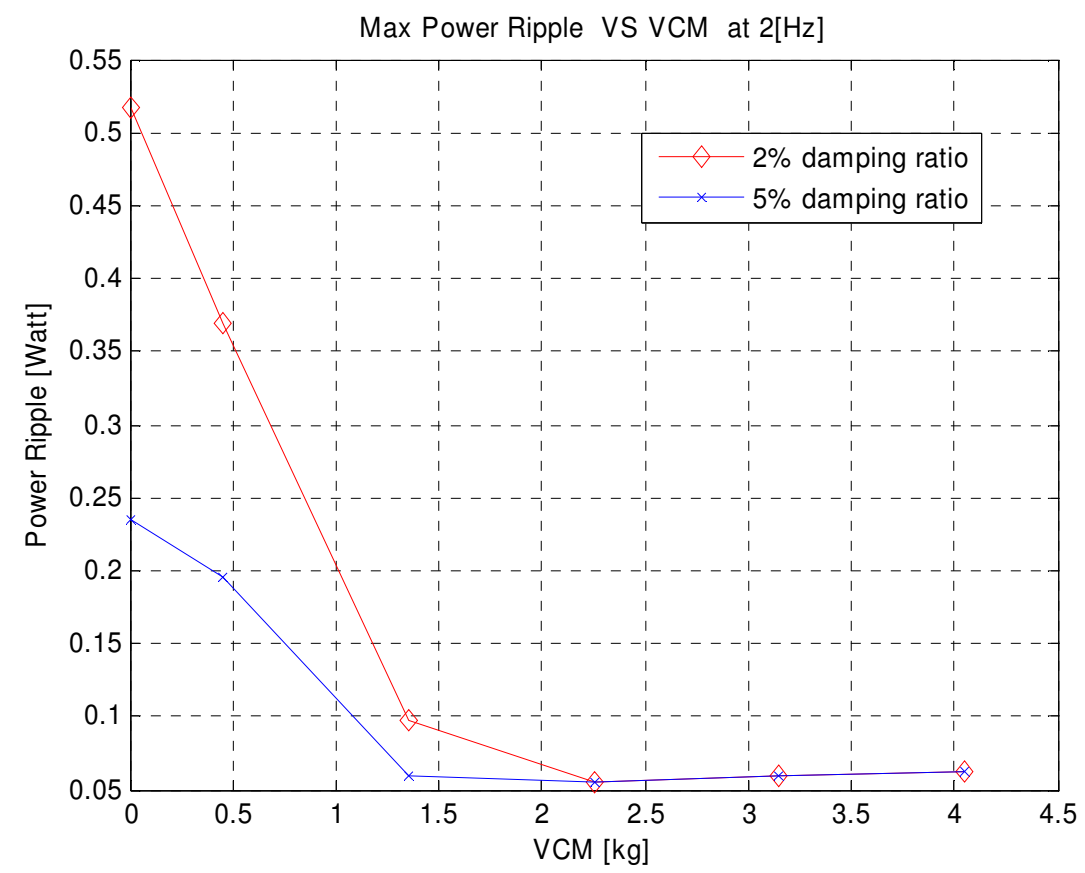

Fig. 6.33 Maximum Power Ripple VS Vibration Control Mass

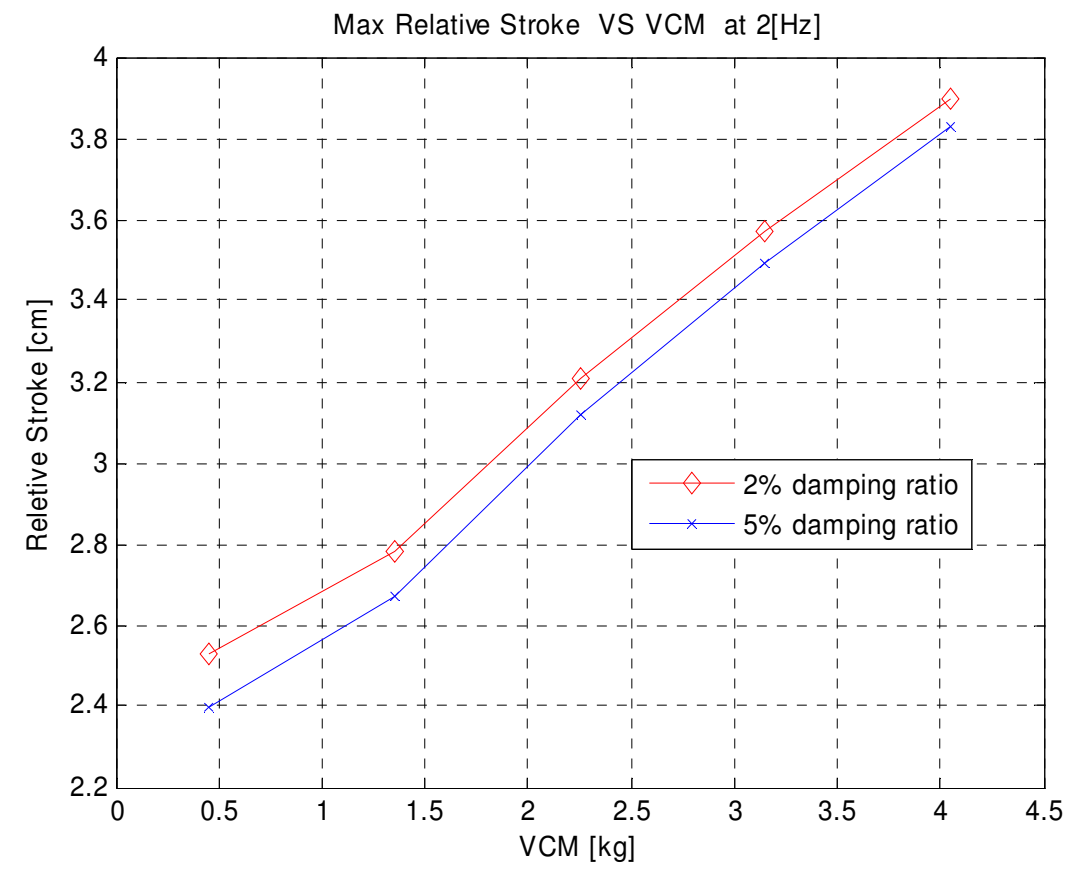

Fig. 6.34 Maximum Relative Stroke of Appendage Vs Vibration Control Mass 


\subsection{Isolation of the Satellite from the Flywheel's Mass Imbalance Forces}

Fig.6.35 thru 6.38 show various of the system responses due to a flywheel imbalance eccentricity of $1 \mathrm{e}-5$ [in] $(=2.54 \mathrm{e}-7[\mathrm{~m}])$ at module 2 . The rigid bearing supported flywheel case, the transmitted imbalance force is approximately $67[\mathrm{~N}]$. Fig.6.35 shows the AMB forces applied to the satellite and the motor torque without the notch and band-pass filters in the AMB suspension controller and without the low-pass filter in the flywheel motor torque control loop. The maximum forces transmitted to satellite are about $2.8[\mathrm{~N}]$ and the maximum torques are about $1.25[\mathrm{~N}-\mathrm{m}]$ for this case. Fig.6.36 and 6.37 show AMB forces and flywheel motor torques with only the notch filter inserted, and with both the notch and the band-pass filters inserted, respectively. The transmitted forces and torques are reduced to $0.55[\mathrm{~N}]$ and $0.258[\mathrm{~N}-\mathrm{m}]$ for the notch filter only case, and $0.0035[\mathrm{~N}]$ and $0.0018[\mathrm{~N}-\mathrm{m}]$ for the notch and band-pass filters inserted case, respectively. Fig.6.38 shows that transmitted forces and torques with the low-pass, band-pass and notch filter (cut off frequency $=100[\mathrm{~Hz}]$ ) systems inserted. The forces are almost the same as Fig.6.37, however, the flywheel motor torques are significantly reduced.

In summary, in the case of rigid bearing suspended flywheel (flywheel has only spin motion) the shaking force due to flywheel imbalance is enormous compared with AMB suspended flywheel case. The filter stages including notch and band pass filters can diminish shaking force almost zero. 

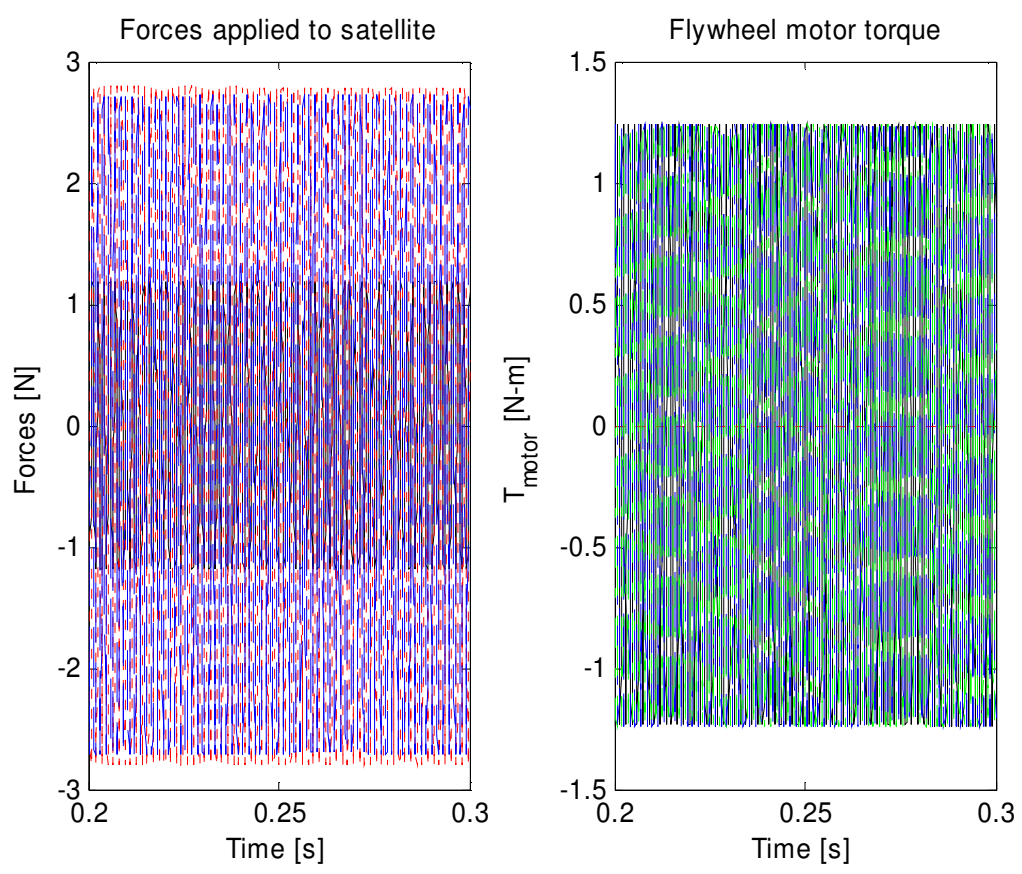

Fig. 6.35 Transmitted Forces and Torques without Notch and Band-Pass Filter
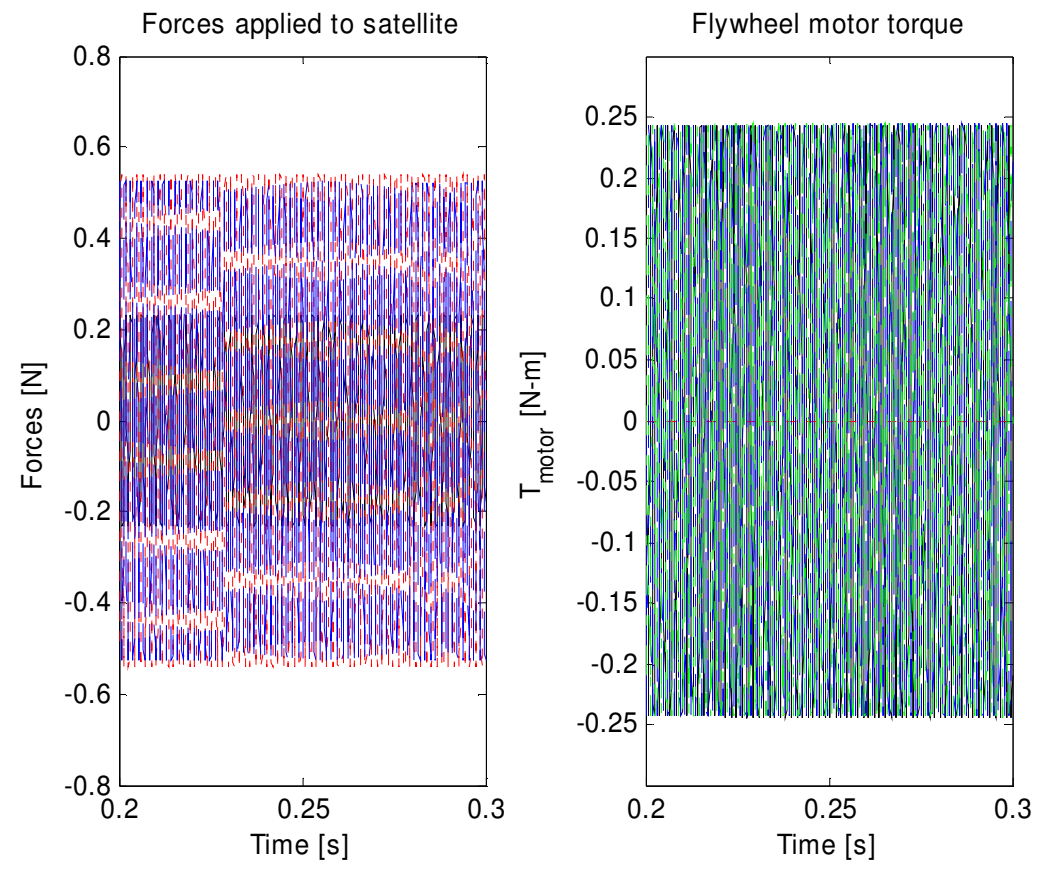

Fig. 6.36 Transmitted Forces and Motor Torques with Notch Filter 

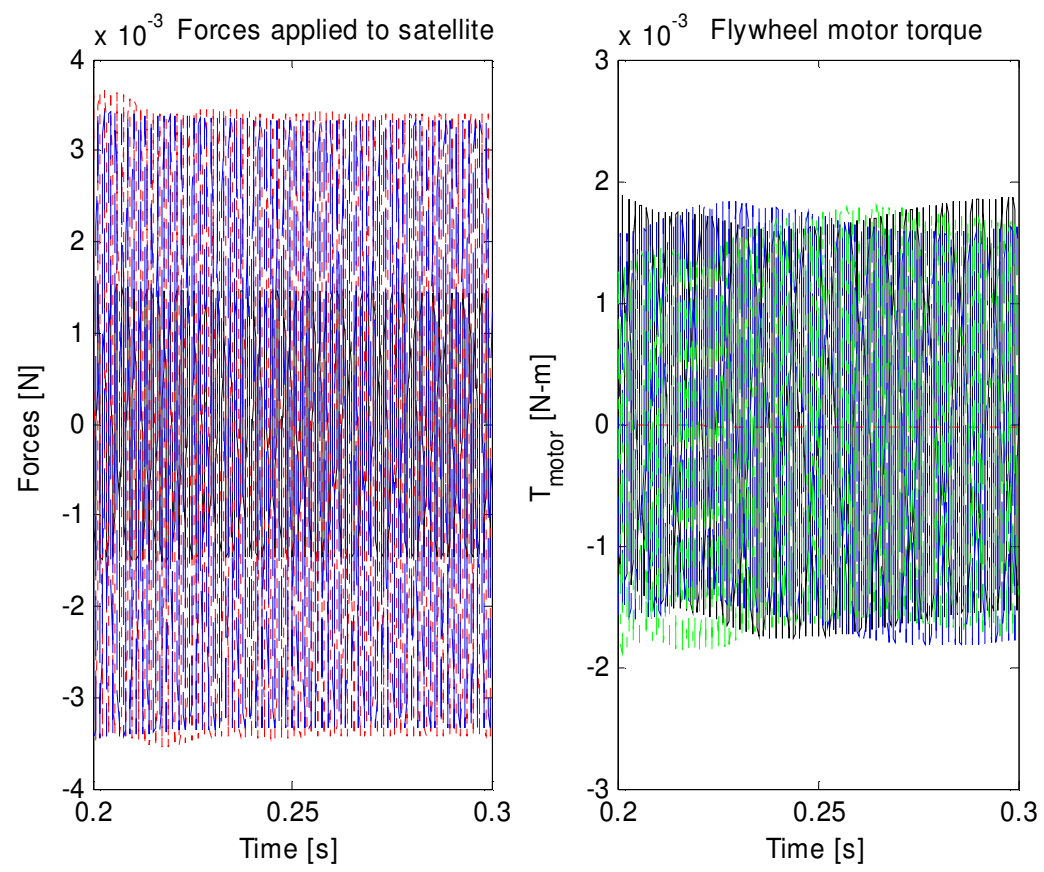

Fig. 6.37 Transmitted Forces and Motor Torques with Notch and Band-Pass Filter
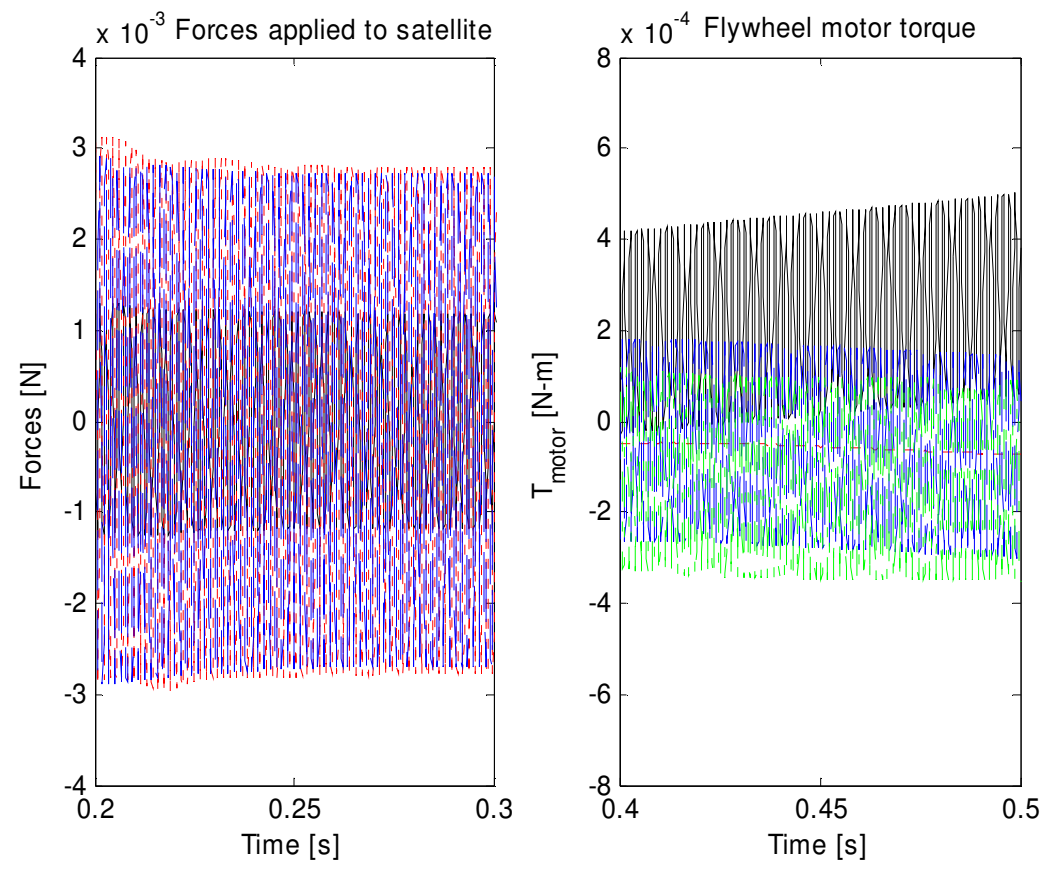

Fig. 6.38 Transmitted Forces and Motor Torques with All Filter Stages 


\section{CHAPTER VII}

\section{IPAC WITH TWO VARIABLE SPEED CONTROL MOMENT}

\section{GYROS}

\subsection{Overview}

This Chapter VII will present IPAC service with two single gimbaled variable speed flywheels. The single gimbaled variable speed flywheel is called as variable speed control moment of gyroscope (VSCMG). Generally speaking, VSCMG combines advantages of the classical single gimbaled control moment of gyro and reaction wheel. The advantages of a control moment gyro (CMG) and reaction wheels (RWs) are that a large effective control torque applied to the spacecraft could be produced by a relatively small gimbal torque input, and do not have singularity configurations and typically have simpler control law than CMG clusters, respectively. On the other hand, the disadvantages are that the single gimbaled CMGs are that their control laws are fairly complex and encounter certain singular gimbal angle configurations and RW systems include a relatively small effective torque being produced on the spacecraft and the possibility of reaction wheel saturation [4]. VSCMGs can produce an extra degree of control to the classical single gimbaled device because the spinning disk can be rotated or gimbaled about a single body fixed axis, while the disk spin rate is also free to be controlled [38, 39].

The dynamics, feedback control law and two different steering laws (velocity based and acceleration based steering laws) of VSCMGs are discussed in [4]. The previous work [5] examines the four simultaneous use of single gimbaled variable speed control 
moment of gyroscopes as spacecraft attitude control actuators and excessive energy storage devices. This present work will demonstrates that the integrated power and attitude control tasks will be achieved by with only two VSCMGs instead of utilizing a standard four VSCMGs pyramid configuration in the literature [5]. The detailed dynamics and control laws are derived in $[4,5]$ concerning IPAC, so they might be briefly reviewed again in this chapter and mostly simulation results will be presented.

\subsection{VSCMGs Dynamics Part}

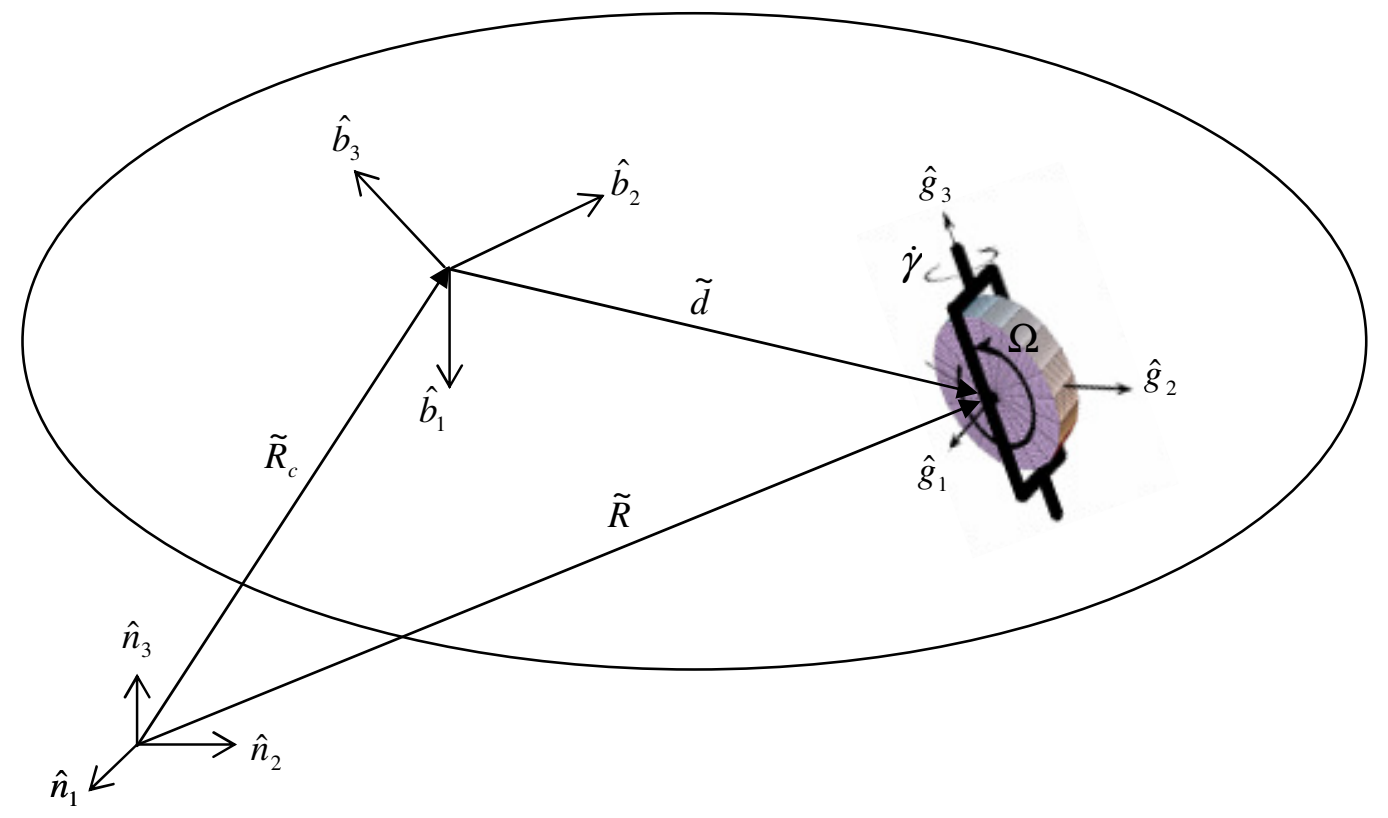

Fig. 7.1 One VSCMG Coordinate System

One variable speed control moment of gyro is shown in the Fig.7.1, and then equation of motion can be written as following. The system angular momentum is sum 
of each component (satellite body, wheel and gimbal) and be expressed as (7.1) and (7.2). The inertial reference, satellite body and gimbal coordinated are denoted as $\hat{n}, \hat{b}$, and $\hat{g}$, respectively. The angular velocities of gimbal respect to body and momentum wheel angular velocity respect to gimbal frame are $\dot{\tilde{\gamma}}=\left[\begin{array}{lll}0 & 0 & \dot{\gamma}\end{array}\right]$ and $\widetilde{\Omega}=\left[\begin{array}{lll}\Omega & 0 & 0\end{array}\right]$, respectively.

$$
\begin{gathered}
\tilde{H}_{s y s}=\tilde{H}_{s c}+\tilde{H}_{w}+\tilde{H}_{g} \\
\tilde{H}_{s c}=I_{s c}^{b} \widetilde{\omega}_{b / n}^{b}, \quad \tilde{H}_{w}=I_{w}^{g} \tilde{\omega}_{w / n}^{g}, \quad \tilde{H}_{g}=I_{g}^{g} \tilde{\omega}_{g / n}^{g}
\end{gathered}
$$

The satellite body inertia matrix shown in (7.2) is satellite body inertia itself plus VSCMG inertia components due to the fact that the mass center of VSCMG is located from the satellite center of mass by a vector $\tilde{d}$. Let's define the inertial time derivative of a vector $A$ is expressed as (7.3.1) and $\vec{\omega}$ is defined as (7.3.2), respectively.

$$
\begin{gathered}
{ }^{N} \frac{d}{d t}(\tilde{A}) \equiv \dot{\tilde{A}} \\
\vec{\omega} \equiv\left[\begin{array}{ccc}
0 & -\omega_{3} & \omega_{2} \\
\omega_{3} & 0 & -\omega_{1} \\
-\omega_{2} & \omega_{1} & 0
\end{array}\right] \text { where } \widetilde{\omega}=\left[\begin{array}{lll}
\omega_{1} & \omega_{2} & \omega_{3}
\end{array}\right]^{T}
\end{gathered}
$$

so, the inertial time derivative of each component and equations of motion of a system of rigid bodies from Euler's equation can be written as (7.4.1) thru (7.4.3) and (7.5), respectively.

$$
\begin{aligned}
& \dot{\tilde{H}}_{s c}=\dot{\tilde{H}}_{s c}^{b}+\vec{\omega}_{b / n}^{b} \tilde{H}_{s c}^{b} \\
& \dot{\tilde{H}}_{w}=C \dot{\tilde{H}}_{w}^{g}+\vec{\omega}_{b / n}^{b} C \tilde{H}_{w}^{g}+C \ddot{\omega}_{g / b}^{g} \tilde{H}_{w}^{g}
\end{aligned}
$$




$$
\dot{\tilde{H}}_{g}=C \dot{\tilde{H}}_{g}^{g}+C \ddot{\omega}_{g / b}^{g} \tilde{H}_{g}^{g}+\overleftrightarrow{\omega}_{b / n}^{b} C \tilde{H}_{g}^{g}
$$

where $C$ is direction cosine matrix (DCM) between satellite body and gimbal frame, $\widetilde{\omega}_{b / n}^{b}$ is satellite body angular velocity coordinatized in body frame $\left(\widetilde{\omega}=\left[\begin{array}{lll}\omega_{1} & \omega_{2} & \omega_{3}\end{array}\right]^{T}\right)$,

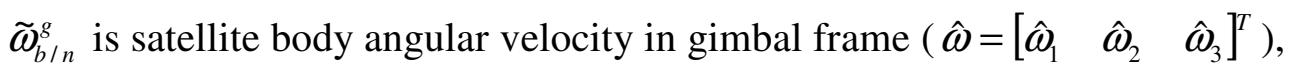

$\widetilde{\omega}_{w / n}^{g}$ is wheel angular velocity coordinatized in the gimbal frame $\left(\widetilde{\omega}_{w / n}^{g}=C^{T} \widetilde{\omega}+\dot{\tilde{\gamma}}+\widetilde{\Omega}\right)$, $\widetilde{\omega}_{g / n}^{g}$ is gimbal angular velocity coordinatized in the gimbal frame $\left(\widetilde{\omega}_{g / n}^{g}=C^{T} \widetilde{\omega}+\dot{\tilde{\gamma}}\right)$, and $\tilde{L}$ is external torque applied to satellite which is assumed $\tilde{L}=\tilde{0}$.

$$
\dot{\tilde{H}}_{s y s}=\dot{\tilde{H}}_{s c}+\dot{\tilde{H}}_{w}+\dot{\tilde{H}}_{g}=\tilde{L}
$$

The equations (7.4.1) thru (7.4.3) can be rewritten as following with some manipulation,

1. The first term of (7.4.1) is expressed as

$\dot{\tilde{H}}_{s c}^{b}=I_{s c}^{b} \dot{\tilde{\omega}}_{b / n}^{b}$

2. Sum of second term of (7.4.1), (7.4.2) and third term of (7.4.3) is also expressed as

$$
\begin{aligned}
\vec{\omega}_{b / n}^{b} \tilde{H}_{s c}^{b}+\vec{\omega}_{b / n}^{b} C \tilde{H}_{w}^{g}+\vec{\omega}_{b / n}^{b} C \tilde{H}_{g}^{g}= & \vec{\omega}_{b / n}^{b}\left(I_{s c}^{b}+I_{w}^{b}\right) \widetilde{\omega}_{b / n}^{b}+\vec{\omega}_{b / n}^{b} C I_{w}^{g}\left[\begin{array}{lll}
\Omega & 0 & \dot{\gamma}
\end{array}\right]^{T}+ \\
& \vec{\omega}_{b / n}^{b} C I_{g}^{g}\left[\begin{array}{lll}
0 & 0 & \dot{\gamma}
\end{array}\right]^{T}+\vec{\omega}_{b / n}^{b} I_{g}^{b} \widetilde{\omega}_{b / n}^{b}= \\
& \vec{\omega}_{b / n}^{b}\left(I_{s c}^{b}+I_{w}^{b}+I_{g}^{b}\right) \widetilde{\omega}_{b / n}^{b}+\vec{\omega}_{b / n}^{b} C I_{w}^{g}\left[\begin{array}{lll}
\Omega & 0 & \dot{\gamma}
\end{array}\right]^{T}+ \\
& \vec{\omega}_{b / n}^{b} C I_{g}^{g} \cdot\left[\begin{array}{lll}
0 & 0 & \dot{\gamma}
\end{array}\right]^{T}
\end{aligned}
$$

3. Sum of first term of (7.4.2) and (7.4.3) is expressed as

$$
\begin{aligned}
& C \dot{\tilde{\tilde{H}}}_{w}^{g}+C \dot{\tilde{\tilde{H}}}_{g}^{g}=C I_{w}^{g}\left[\begin{array}{lll}
\dot{\Omega} & 0 & \ddot{\gamma}
\end{array}\right]^{T}+C I_{w}^{g} C^{T} \ddot{\omega}_{b / g}^{b} \widetilde{\omega}_{b / n}^{b}+I_{w}^{b} \dot{\tilde{\omega}}_{b / n}^{b}+ \\
& C I_{g}^{g}\left[\begin{array}{lll}
0 & 0 & \ddot{\gamma}
\end{array}\right]^{T}+C I_{g}^{g} C^{T} \vec{\omega}_{b / g}^{b} \tilde{\omega}_{b / n}^{b}+I_{g}^{b} \dot{\tilde{\omega}}_{b / n}^{b}
\end{aligned}
$$

4. Sum of third term of (7.4.2) and second term of (7.4.3) is expressed as 


$$
\begin{aligned}
& C \ddot{\omega}_{g / b}^{g} \tilde{H}_{w}^{g}+C \ddot{\omega}_{g / b}^{g} \tilde{H}_{g}^{g}=C \ddot{\omega}_{g / b}^{g} I_{w}^{g}\left[\begin{array}{lll}
\Omega & 0 & \dot{\gamma}
\end{array}\right]^{T}+C \vec{\omega}_{g / b}^{g} I_{w}^{g} C^{T} \widetilde{\omega}_{b / n}^{b}
\end{aligned}
$$

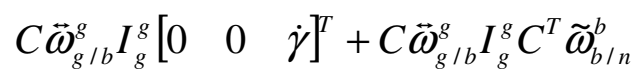

Therefore, the total equation of motion can be obtained by gathering from (7.6.1) thru (7.6.4) as shown by (7.7.1) in the satellite body coordinate.

$$
\begin{aligned}
& \left(I_{s c}^{b}+I_{w}^{b}+I_{g}^{b}\right) \dot{\tilde{\omega}}_{b / n}^{b}+\vec{\omega}_{b / n}^{b}\left(I_{s c}^{b}+I_{w}^{b}+I_{g}^{b}\right) \tilde{\omega}_{b / n}^{b}+ \\
& C\left\{\begin{array}{l}
\ddot{\omega}_{b / n}^{g} I_{w}^{g}\left[\begin{array}{lll}
\Omega & 0 & \dot{\gamma}
\end{array}\right]^{T}+\vec{\omega}_{b / n}^{g} I_{g}^{g}\left[\begin{array}{lll}
0 & 0 & \dot{\gamma}
\end{array}\right]^{T}+I_{w}^{g}\left[\begin{array}{lll}
\dot{\Omega} & 0 & \ddot{\gamma}
\end{array}\right]^{T}-I_{w}^{g} \vec{\omega}_{g / b}^{g} \widetilde{\omega}_{b / n}^{g}+ \\
I_{g}^{g}\left[\begin{array}{lll}
0 & 0 & \ddot{\gamma}
\end{array}\right]^{T}-I_{g}^{g} \vec{\omega}_{g / b}^{g} \omega_{b / n}^{g}+\vec{\omega}_{g / b}^{g} I_{w}^{g}\left[\begin{array}{lll}
\Omega & 0 & \dot{\gamma}
\end{array}\right]^{T}+\ddot{\omega}_{g / b}^{g} I_{w}^{g} C^{T} \omega_{b / n}^{b}+ \\
\vec{\omega}_{g / b}^{g} I_{g}^{g}\left[\begin{array}{lll}
0 & 0 & \dot{\gamma}
\end{array}\right]^{T}+\vec{\omega}_{g / b}^{g} I_{g}^{g} C^{T} \omega_{b / n}^{b}
\end{array}\right\}=\tilde{L} \\
& I \dot{\tilde{\omega}}_{b / n}^{b}+\ddot{\omega}_{b / n}^{b} I \tilde{\omega}_{b / n}^{b}+C F=\tilde{L} \\
& \text { where } F=\left\{\begin{array}{c}
\left(J_{t}+J_{3}\right) \dot{\gamma} \hat{\omega}_{2}+\left(J_{a}+J_{1}\right) \dot{\gamma} \hat{\omega}_{2}+J_{a} \dot{\Omega}-\left(J_{t}+J_{2}\right) \dot{\gamma} \hat{\omega}_{2} \\
J_{a} \Omega \hat{\omega}_{3}-\left(J_{t}+J_{3}\right) \dot{\gamma} \hat{\omega}_{1}-\left(J_{t}+J_{2}\right) \dot{\gamma} \hat{\omega}_{1}+J_{a} \Omega \dot{\gamma}+\left(J_{a}+J_{1}\right) \dot{\gamma} \hat{\omega}_{1} \\
-J_{a} \Omega \hat{\omega}_{2}+\left(J_{t}+J_{3}\right) \ddot{\gamma}
\end{array}\right\} \\
& I_{w}^{g}=\operatorname{diag}\left(J_{a}, J_{t}, J_{t}\right), I_{g}^{g}=\operatorname{diag}\left(J_{1}, J_{2}, J_{3}\right) \text { and } I=I_{s c}^{b}+I_{w}^{b}+I_{g}^{b}
\end{aligned}
$$

$F$ term in the (7.7.2) can be simplified as combining the inertia matrix of the RW and the gimbal frame into VSCMG inertia matrix $J$. and rewritten as (7.7.4) with $J_{S} \approx J_{a}$ assumption.

$$
\begin{gathered}
J=I_{w}^{g}+I_{g}^{g}=\operatorname{diag}\left(J_{S}, J_{T}, J_{G}\right) \\
F=\left\{\begin{array}{c}
J_{S}\left(\dot{\gamma} \hat{\omega}_{2}+\dot{\Omega}\right)-\left(J_{T}-J_{G}\right) \dot{\gamma} \hat{\omega}_{2} \\
J_{S}\left(\Omega+\hat{\omega}_{1}\right) \dot{\gamma}+J_{S} \Omega \hat{\omega}_{3}-\left(J_{T}+J_{G}\right) \dot{\gamma} \hat{\omega}_{1} \\
J_{G} \ddot{\gamma}-J_{S} \Omega \hat{\omega}_{2}
\end{array}\right\}
\end{gathered}
$$

Now, the equation (7.7.2) can be extended as following utilized by results of (7.7.4).

$$
I \dot{\tilde{\omega}}_{b / n}^{b}+\vec{\omega}_{b / n}^{b} I \tilde{\omega}_{b / n}^{b}+B \ddot{\gamma}+D_{S} \dot{\gamma}+E \dot{\Omega}+F \Omega=\tilde{L}
$$


, where $B=\left[J_{G} C_{1}^{3}\right]$

$$
\begin{aligned}
& D_{S}=\left[\left(J_{S} \hat{\omega}_{2}-J_{P} \hat{\omega}_{2}\right) C_{1}^{1}+J_{S}\left(\hat{\omega}_{1}+\Omega_{1}\right) C_{1}^{2}-J_{Q} \hat{\omega}_{1} C_{1}^{2}\right] \\
& E=\left[J_{S} C_{1}^{1}\right] \\
& F=\left[\hat{\omega}_{3} C_{1}^{2}-J_{S} \hat{\omega}_{2} C_{1}^{3}\right]
\end{aligned}
$$

\subsection{VSCMGs Control Law Part}

The feedback control law utilized for variable speed control moment gyro is identical with that used in the Chapter III. The following Lyapunov function $V$ is a positive definite, radially unbounded measure of the total system state error relative to the target state where $k_{l}$ is a scalar attitude feedback gain.

$$
V(\delta \widetilde{\omega}, \delta \widetilde{\sigma})=\frac{1}{2} \delta \widetilde{\omega}^{T} I \delta \widetilde{\omega}+2 k_{1} \log \left(1+\delta \tilde{\sigma}^{T} \delta \widetilde{\sigma}\right)
$$

The first term of (7.8) can be rewritten as (7.9.1) due to time varying of VSCMG inertia matrix. The inertia matrix $I$ is defined as $I=I_{s c}^{b}+I_{w}^{b}+I_{g}^{b}=I_{s c}^{b}+J^{b}$ before where $J^{b}=I_{w}^{b}+I_{g}^{b}$ and the time derivative of (7.8) is expressed as (7.9.2). The time derivative of second term in (7.8) is derived in the Chapter III.

$$
\begin{gathered}
\frac{1}{2} \delta \tilde{\omega}^{T} I \delta \widetilde{\omega}=\frac{1}{2} \delta \tilde{\omega}^{T}\left(I_{s c}^{b}+J^{b}\right) \delta \tilde{\omega} \\
\dot{V}(\delta \tilde{\omega}, \delta \tilde{\sigma})=\delta \tilde{\omega}^{T} I \delta \dot{\tilde{\omega}}+\frac{1}{2} \delta \tilde{\omega}^{T} \dot{J}^{b} \delta \widetilde{\omega}+k_{1} \delta \tilde{\omega}^{T} \delta \tilde{\sigma} \\
=\delta \widetilde{\omega}^{T}\left(I \delta \dot{\tilde{\omega}}+\frac{1}{2} \dot{J}^{b} \delta \widetilde{\omega}+k_{1} \delta \tilde{\sigma}\right)
\end{gathered}
$$

Lyapunov stability theory requires that $\dot{V}$ be negative semi-definite to guarantee stability, let $k_{2}$ is a positive definite angular velocity feedback gain, then (7.9.2) can be 
expressed as (7.9.3) and (7.9.4).

$$
\begin{gathered}
\dot{V}(\delta \omega, \delta \sigma)=-\delta \widetilde{\omega}^{T} k_{2} \delta \omega \\
I \delta \dot{\tilde{\omega}}+\frac{1}{2} \dot{J}^{b} \delta \widetilde{\omega}+k_{1} \delta \widetilde{\sigma}=-k_{2} \delta \widetilde{\omega} \text { where } \delta \widetilde{\omega}=\widetilde{\omega}-\widetilde{\omega}_{r}
\end{gathered}
$$

The equation (7.9.4) can be rearranged by plugging (7.7.5) into (7.9.4) as following,

$$
B \ddot{\gamma}+D_{S} \dot{\gamma}+E \dot{\Omega}+F \Omega=\tilde{L}-I \dot{\widetilde{\omega}}_{r}+\frac{1}{2} \dot{J}^{b} \delta \widetilde{\omega}+k_{1} \delta \widetilde{\sigma}+k_{2} \delta \widetilde{\omega}-\overleftrightarrow{\omega} I \omega
$$

As mentioned above, the external torque vector, $\tilde{L}$ is zero and the third term of right hand side in the (7.9.5) can be expressed as followings,

$$
\begin{aligned}
\frac{1}{2} \dot{J}^{b} \delta \widetilde{\omega} & =\frac{1}{2} C\left[\vec{\omega}_{g / b}^{g} J^{g}-J^{g} \ddot{\omega}_{g / b}^{g}\right] C^{T} \delta \omega \\
& =\frac{1}{2} C\left[\vec{\omega}_{g / b}^{g} J^{g}-J^{g} \ddot{\omega}_{g . b}^{g}\right] \delta \hat{\omega} \\
& =R \dot{\gamma}
\end{aligned}
$$

where $R=\frac{1}{2}\left[J_{r} \delta \hat{\omega}_{2} C_{1}^{1}+J_{r} \delta \hat{\omega}_{1} C_{1}^{2}\right], \delta \hat{\omega}=C^{T} \delta \widetilde{\omega}=\delta \omega^{g}$ and $J_{r}=J_{S}-J_{T}$

The following relationship (7.9.7) can be found by substituting (7.9.6) into (7.9.5)

$$
B \ddot{\gamma}+D \dot{\gamma}+E \dot{\Omega}+F \Omega=-I \dot{\tilde{\omega}}_{r}+k_{1} \delta \tilde{\sigma}+k_{2} \delta \widetilde{\omega}-\overleftrightarrow{\omega} I \omega
$$

where $D=D_{S}-R$ and as usual if the gimbal acceleration is assumed to be small, the first term of (7.9.7) can be ignored, then it can be rearranged as (7.9.8)

$$
D \dot{\gamma}+E \dot{\Omega}=\tilde{L}_{r}-F \Omega
$$

where $\tilde{L}_{r}=-I \dot{\tilde{\omega}}_{r}+k_{1} \delta \tilde{\sigma}+k_{2} \delta \tilde{\omega}-\vec{\omega} I \omega$ is required attitude control torque. 


\subsection{VSCMGs Torque Distributions Part}

In this section, attitude control and power transfer torques will be presented. Most required torques produced by a combination of the RW angular velocity $(\dot{\Omega})$ and gimbal rate $(\dot{\gamma})$ not gimbal acceleration $(\ddot{\gamma})$ are desirable to amplify the potential torques

The total kinetic energy stored in the reaction wheel is

$$
E_{R W}=\frac{1}{2} \widetilde{\Omega}^{T} J_{R W} \tilde{\Omega}
$$

Hence, the rate of change of the energy (power) is given by

$$
\dot{E}_{R W}=P_{R W}=\left[\widetilde{\Omega}^{T} J_{R W} \quad 0_{1 \times 2}\left[\begin{array}{c}
\dot{\tilde{\Omega}} \\
\dot{\tilde{\gamma}}
\end{array}\right]\right.
$$

where $J_{R W}=\operatorname{diag}\left(J_{S}^{1}, J_{S}^{2}\right)$, therefore simultaneous attitude control and power transfer constraint equation can be obtained by combining (7.9.8) and (7.11).

$$
\left[\begin{array}{ll}
Q_{11} & Q_{12} \\
Q_{21} & Q_{22}
\end{array}\right] \eta=\left[\begin{array}{c}
\tilde{L}_{T} \\
P_{R W}
\end{array}\right]
$$

where $Q_{11}=E, \quad Q_{12}=D, \quad Q_{21}=\tilde{\Omega}^{T} J_{R W}, \quad Q_{22}=0_{1 \times 2}, \quad \eta=\lfloor\dot{\tilde{\Omega}} \quad \dot{\tilde{\gamma}}\rfloor, \quad \tilde{L}_{T}=\tilde{L}_{r}-F \Omega$ As defining $Q_{1}=\left[\begin{array}{ll}Q_{11} & Q_{12}\end{array}\right], Q_{2}=\left[\begin{array}{ll}Q_{21} & Q_{22}\end{array}\right]$, required attitude control torque constraint becomes (7.13) and $Q_{1}=3 \times 4, \eta=4 \times 1$ matrices.

$$
Q_{1} \eta=\tilde{L}_{T}
$$

The general solution to (7.13) is given by

$$
\eta=Q_{1}^{+} \tilde{L}_{T}+\eta_{\text {null }}
$$

where $Q_{1}^{+}$is general inverse matrix of $Q_{1}$ which obtained from range space of $Q_{1}$ and 
$\eta_{\text {null }}$ is null vector which obtained from null space of $Q_{1}$ (i.e. $Q_{1} \eta_{\text {null }}=0$ ).

Required power transfer control torque constraint becomes (7.15) and $Q_{2}=1 \times 4, \eta=4 \times 1$ matrices. After substitute (7.14) into (7.15), it yields (7.16) and the null vector, $\eta_{\text {null }}$ can be obtained from it.

$$
\begin{gathered}
Q_{2} \eta=P_{R W} \\
Q_{2}\left(Q_{1}^{+} \tilde{L}_{T}+\eta_{\text {null }}\right)=P_{R W}
\end{gathered}
$$

Let define modified power $P_{m}=P_{R W}-Q_{2} Q_{1}^{+} \tilde{L}_{T}=Q_{2} \eta_{\text {null }}$. As discussed in the Chapter III, the null vector, $\eta_{\text {null }}$ is obtained from null space of $Q_{1}$, so there exists a vector satisfying

$$
\eta_{\text {null }}=P_{N} v
$$

where $P_{N}$ is the orthogonal projection onto null space of $Q_{1}$ and property of $P_{N} P_{N}^{T}=1$

and $P_{N}=I_{n}-Q_{1}^{+} Q_{1}=I_{n}-Q_{1}^{T}\left(Q_{1} Q_{1}^{T}\right)^{-1} Q_{1}$, then insert (7.17) into modified power equation, $P_{m}=Q_{2} \eta_{\text {null }}$. It yields (7.18).

$$
Q_{2} P_{N} v=P_{m}
$$

and can be rewritten as following using by minimum norm solution

$$
v=\left(Q_{2} P_{N}\right)^{T}\left(Q_{2} P_{N} P_{N}^{T} Q_{2}^{T}\right)^{-1} P_{m}=P_{N}^{T} Q_{2}^{T}\left(Q_{2} P_{N} Q_{2}^{T}\right)^{-1} P_{m}
$$

Therefore, the power transfer torque can be given by (7.20).

$$
\eta_{\text {null }}=P_{N} Q_{2}^{T}\left(Q_{2} P_{N} Q_{2}^{T}\right)^{-1} P_{m}
$$

Finally, the simultaneous attitude control and power transfer torque can be presented as combining (7.14) and (7.20). 


$$
\eta=Q_{1}^{+} \tilde{L}_{T}+P_{N} Q_{2}^{T}\left(Q_{2} P_{N} Q_{2}^{T}\right)^{-1} P_{m}
$$

In this presented work, the weighted generalized inverse $Q_{1}^{+}$is utilized which is given as [4].

$$
Q_{1}^{+}=W Q_{1}^{T}\left(Q_{1} W Q_{1}^{T}\right)^{-1}
$$

where $W$ is a diagonal RW/CMG mode weighting matrix, $W=\operatorname{diag}\left(W_{s}, W_{g}\right)$ where $W_{s}$ and $W_{g}$ are reaction wheel and CMG weighting factor, respectively, which is given by $W_{s}=\operatorname{diag}\left(W_{s 1}, W_{s 2}\right), W_{g}=\operatorname{diag}\left(W_{g 1}, W_{g 2}\right)$. The reaction wheel mode weight, $W_{s}$ is defined as $W_{s}=W_{S i}^{0} \exp (-\mu \delta)$ where $W_{S i}^{0}$ and $\mu$ are positive scalars to be chosen by the control designer and $\delta$ is factor of proximity of singularity which indicates that the gimbal angles approach a singularity $\mathrm{CMG}$ configuration, this parameter will go to zero.

$$
\delta=\operatorname{det}\left(Q_{1} Q_{1}^{T}\right)
$$

\subsection{VSCMGs Simulation Results}

From the previous work [5], it is assured that four VSCMGs can achieve attitude control and power transfer functions at the same time. In this section, the near minimum time rest to rest reference motion is designed as same manner as previous Chapter III and target power transfer is assigned to 1000 [watt] for 30 [sec] during attitude control. The numerical simulations of both four VSCMGs and two VSCMGs are presented in this section with same satellite moment of inertia utilized in the Chapter VI.

The four VSCMGs in a pyramid configuration are described in the [4] and Fig.7.2 shows the two VSCMGs configuration which is removed third and fourth variable speed control moment of gyro from [4]. Table 7.1 presents those simulation parameters for 
both four and two cases. The different results between four and two cases are compared in the next section.

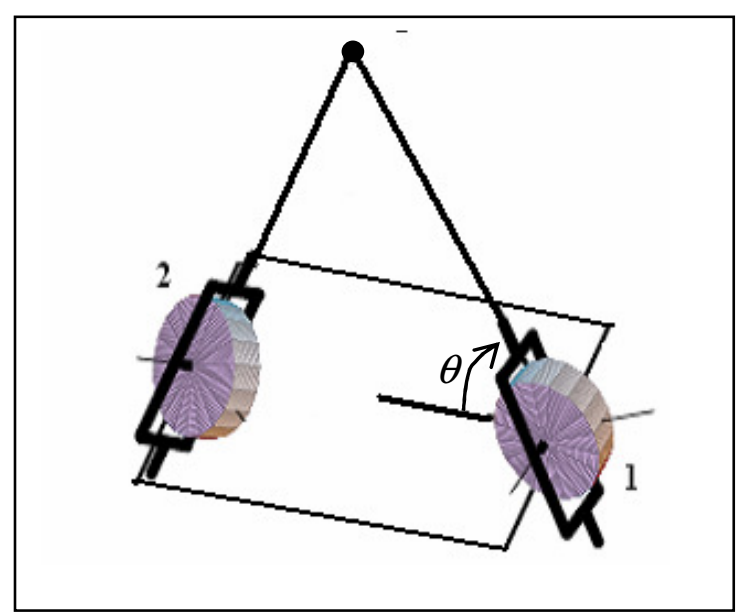

Fig. 7.2 Two VSCMGs IPAC Service Configuration

The angle $\theta$ in the Fig. 7.2 represents the angle of each VSCMG that is measured from the satellite/spacecraft body axis $\left(\hat{b}_{1}-\hat{b}_{2}\right)$ plane to the VSCMG's gimbal axis and the initial gimbal angles are $45[\mathrm{deg}]$ and $-45[\mathrm{deg}]$, respectively. The same initial attitude and velocity errors are used to achieve same satellite motion results with four tetrahedral array flywheels case presented in the previous Chapter VI. The parameter, $\mu$, is selected in a sense of flywheel (reaction wheel) weighting factor $\left(W_{s i}\right)$ will not be zero. In this simulation, a weighted pseudo inverse is used instead of standard Moore- Penrose inverse to obtain solution in (7.13) because ideally the VSCMGs are to act like classical CMGs. If the parameter $\mu$ is chosen to make $W_{s i}$ to be zero, flywheel spin accelerations will be zero, in other words, the flywheel spin velocities are constant which recovered to 
classical CMGs behavior (reaction wheel speed is constant and only gimbal angle rate is changed). Therefore, the parameter $(\mu)$, which can be selected by the control engineer, should be chosen to make $W_{s i}$ to be non-zero value. The time varying $W_{s i}$ is plotted in the following and the gimbal weighting factor $W_{g i}$ is chosen constant value during IPAC.

Table 7.1 VSCMG Simulation Parameters

\begin{tabular}{|c|c|c|c|}
\hline \multirow{2}{*}{ Parameter } & \multicolumn{2}{|c|}{ Value } & \multirow{2}{*}{ Units } \\
\hline & Four VSCMGs & Two VSCMGs & \\
\hline$N$ & 4 & 2 & \\
\hline$\theta$ & 54.75 & 54.75 & [deg] \\
\hline$\omega(0)$ & {$\left[\begin{array}{lll}0 & 0 & 0\end{array}\right]$} & {$\left[\begin{array}{lll}0 & 0 & 0\end{array}\right]$} & {$[\mathrm{rad} / \mathrm{sec}]$} \\
\hline$\sigma(0)$ & {$\left[\begin{array}{lll}-0.025 & 0.0375 & 0\end{array}\right]$} & {$\left[\begin{array}{lll}-0.025 & 0.0375 & 0\end{array}\right]$} & \\
\hline$\gamma(0)$ & {$\left[\begin{array}{llll}45 & -45 & -45 & 45\end{array}\right]$} & {$[45-45]$} & [deg] \\
\hline$\dot{\gamma}(0)$ & {$\left[\begin{array}{llll}0 & 0 & 0 & 0\end{array}\right]$} & {$\left[\begin{array}{ll}0 & 0\end{array}\right]$} & {$[\mathrm{rad} / \mathrm{s}]$} \\
\hline$\Omega(0)$ & {$\left[\begin{array}{llll}4 & 4 & 4 & 4\end{array}\right]^{*} 1 \mathrm{e}+4$} & {$[4]^{*} 1 \mathrm{e}+4$} & {$[\mathrm{rpm}]$} \\
\hline$W_{s i}^{0}$ & 2 & 2 & \\
\hline$W_{g i}$ & 1 & 1 & \\
\hline$J$ & $\operatorname{diag}\left[\begin{array}{llll}0.07 & 0.04 & 0.03\end{array}\right]$ & $\operatorname{diag}\left[\begin{array}{llll}0.07 & 0.04 & 0.03\end{array}\right]$ & {$\left[\mathrm{kg}-\mathrm{m}^{2}\right]$} \\
\hline$k_{2}$ & 1.74 & 1.74 & {$\left[\mathrm{~kg}-\mathrm{m}^{2} / \mathrm{sec}\right]$} \\
\hline$k_{1}$ & 13.2 & 13.2 & {$\left[\mathrm{~kg}-\mathrm{m}^{2} / \mathrm{sec}^{2}\right]$} \\
\hline$\mu$ & $1 e-24$ & $1 e-24$ & \\
\hline
\end{tabular}

\subsubsection{IPAC Simulation Results with Two VSCMGs}

The satellite rotational angle, attitude and velocity vectors are shown in the Fig. 7.3. The target rotational angle (dashed line) is compared with actual rotational angle (solid line) which has initially 10 [deg] errors and the latter tracks almost completely after 18[sec]. Fig. 7.4 is plotted of attitude and velocity error vectors and both of them 
diminished to zero after 30 [sec] later. To perform the desired VSCMGs motion, the weight factors are depending on the proximity to a single gimbal CMG singularity. The scalar factor determined in (7.23) will go to zero when the gimbal angles approach a singular CMG configuration. Fig.7.5 presents the proximity of singularity in two VSCMGs case and it does not go to zero during whole maneuver time. The second plot in the Fig. 7.5 shows the flywheel and gimbal weighting factors $\left(W_{s i}, W_{g i}\right)$, respectively.

The flywheel factor $\left(W_{s i}\right)$ is time varying parameter which shows almost close to 2 during whole maneuver time and gimbal factor $\left(W_{g i}\right)$ is selected to be constant value in the Table 7.1. The gimbal angles and angle rates are shown in the Fig. 7.6. Initially the angles are $45[\mathrm{deg}]$ and $-45[\mathrm{deg}]$ for each gimbal and they reached about $-20[\mathrm{deg}]$ and $275[\mathrm{deg}]$ at the final time, respectively. The second gimbal angle rate is dramatically increased from $-0.05[\mathrm{rad} / \mathrm{s}]$ to $-1.4[\mathrm{rad} / \mathrm{s}]$ at $45[\mathrm{sec}]$. Flywheel spin velocities and accelerations are presented in the Fig. 7.7 and they changed very much compared with four VSCMGs case. The final spin velocities are about 55000[rpm] and 10000[rpm], respectively. The target power (' $\mathrm{x}$ ' mark) and actual power (solid line) schedules are plotted in the Fig. 7.8. The target power is designed to transfer 1000[watt] for 30[sec] of charging case and it can be recognized that the actual power tracks target power successfully. 

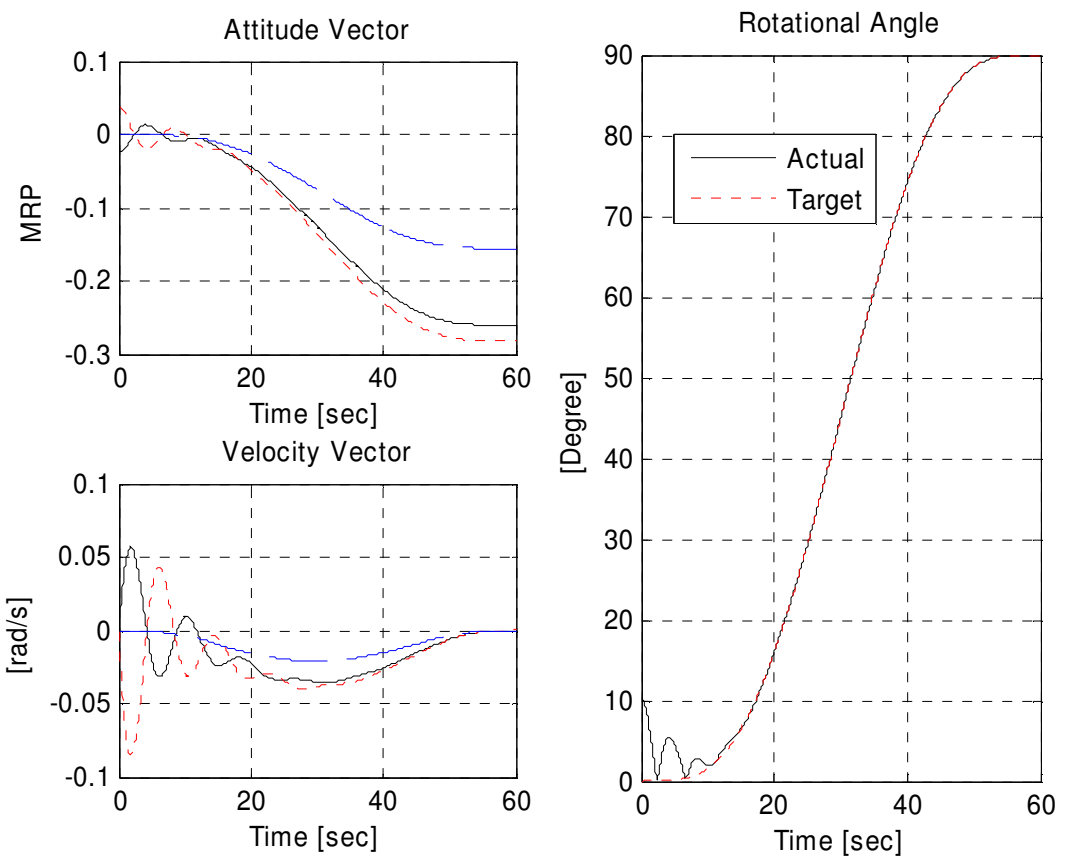

Fig. 7.3 Satellite Motions with Two VSCMGs

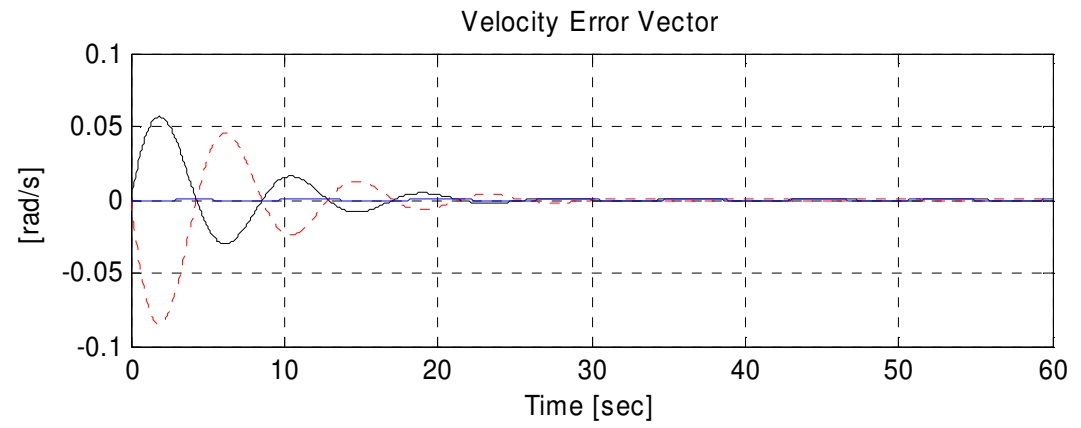

Attitude Error Vector

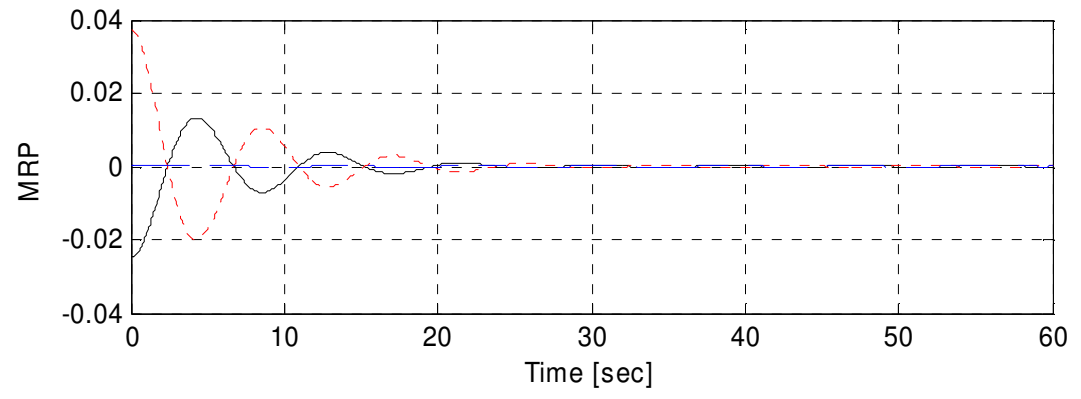

Fig. 7.4 Satellite Error Motions 

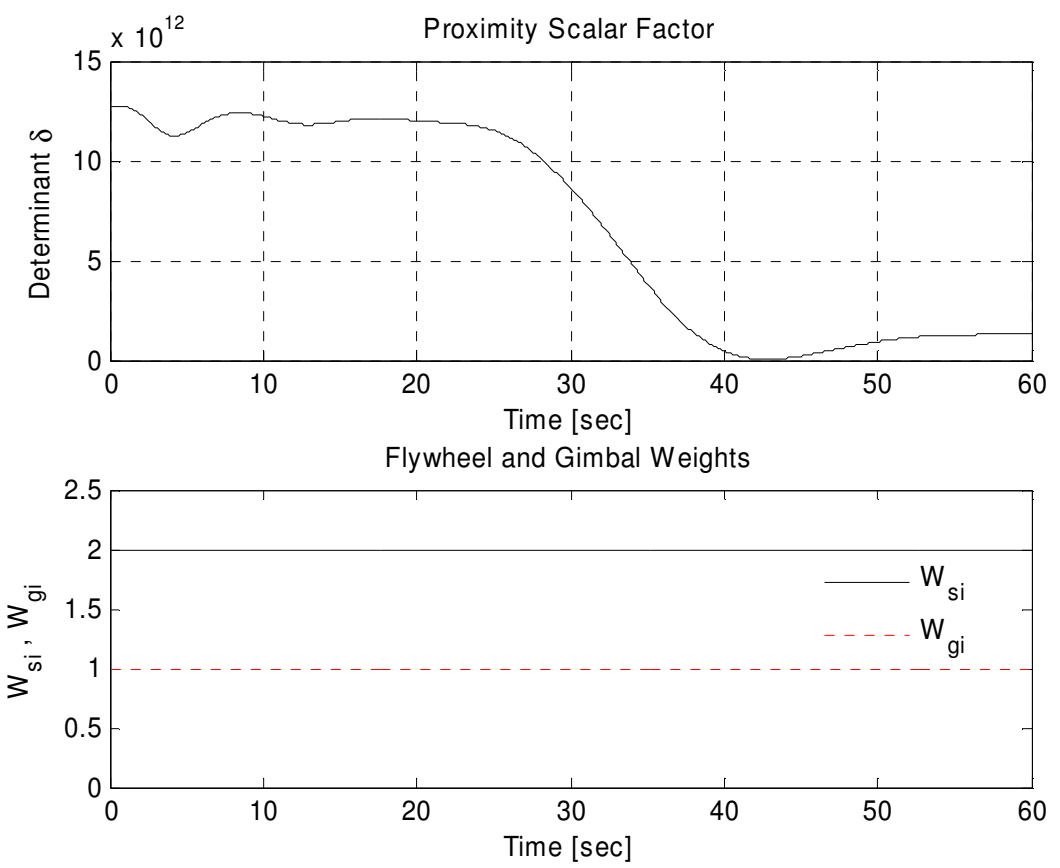

Fig. 7.5 Proximity Scalar and Weights Factor
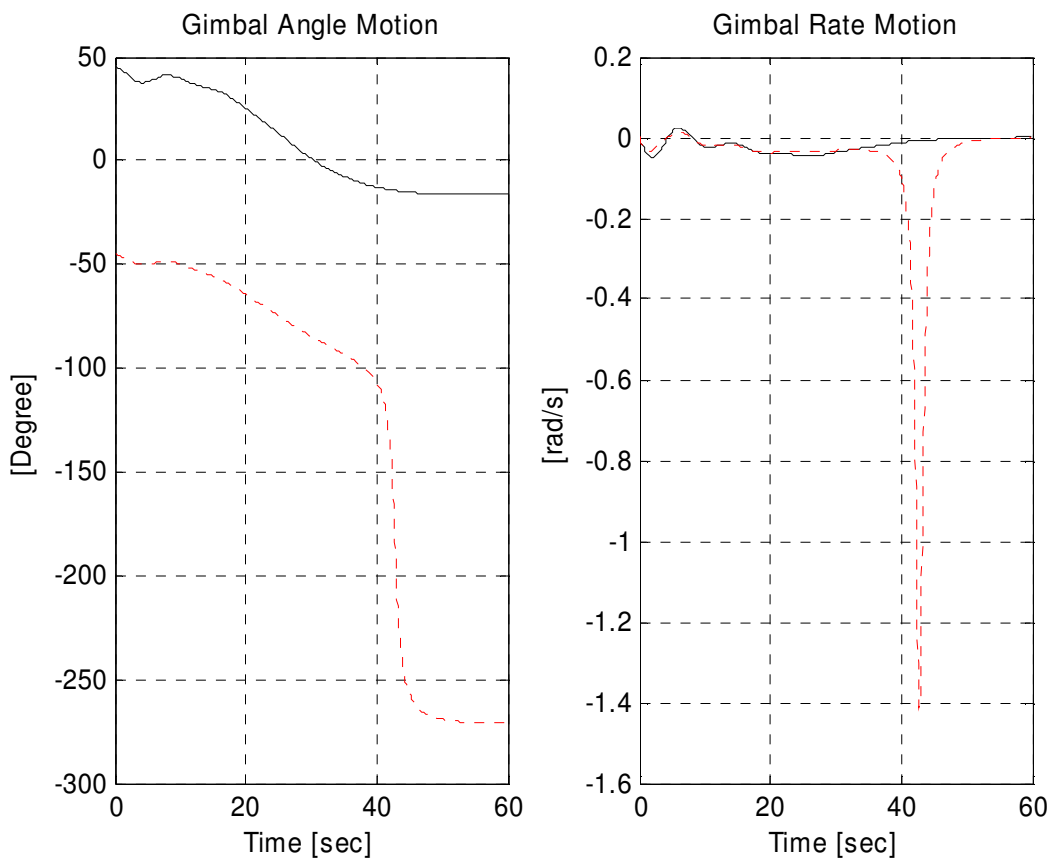

Fig. 7.6 Gimbal Motions 

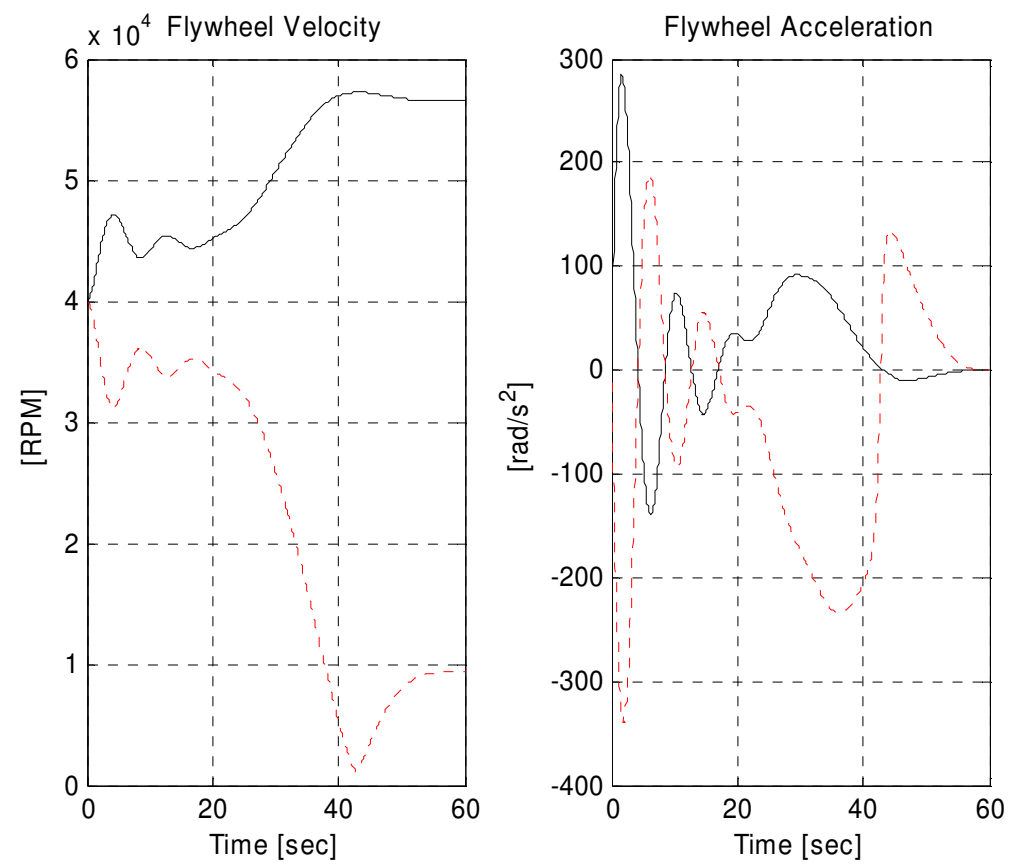

Fig. 7.7 Flywheels (RWs) Motions

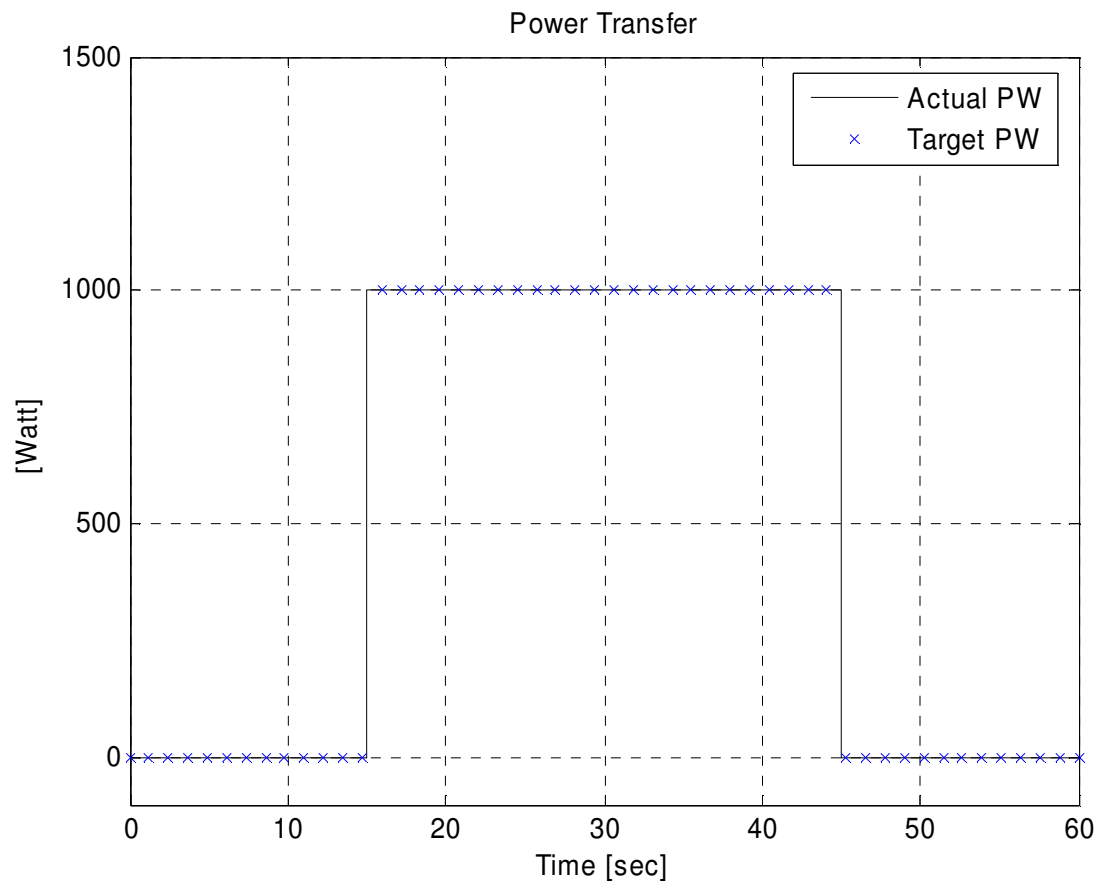

Fig. 7.8 Power Transfer during Attitude Control 


\subsubsection{IPAC Simulation Results with Four VSCMGs}

The following Figs are presented the same attitude control and power tracking tasks with two VSCMGs instead of four. The satellite rotational angle, attitude and angular velocity are identical with two VSCMGs case. The proximity scalar factor shown in the Fig. 7.11 never closes to zero which indicates approach gimbal singularity.

Four different gimbal angles and rates are plotted in the Fig. 7.12 which are little change compared with two gimbal case. The maximum gimbal rate is about $0.5[\mathrm{rad} / \mathrm{s}]$ at the beginning of power tracking task starts. The four flywheels spin speeds and accelerations are shown in the Fig. 7.13. Flywheels spin speed change is very smaller than two gimbal case. In the four VSCMGs case, the attitude control and power tracking variables have 8 (4 flywheel spin speeds and 4 gimbal rates) compared with two VSCMGs case which has only 4 (2 flywheel spin speeds and 2 gimbal rates). These 8 variables can be divided to produce 3 required IPAC torques. Power tracking during attitude maneuver is presented in the Fig. 7.14 and identical with two VSCMGs case. 

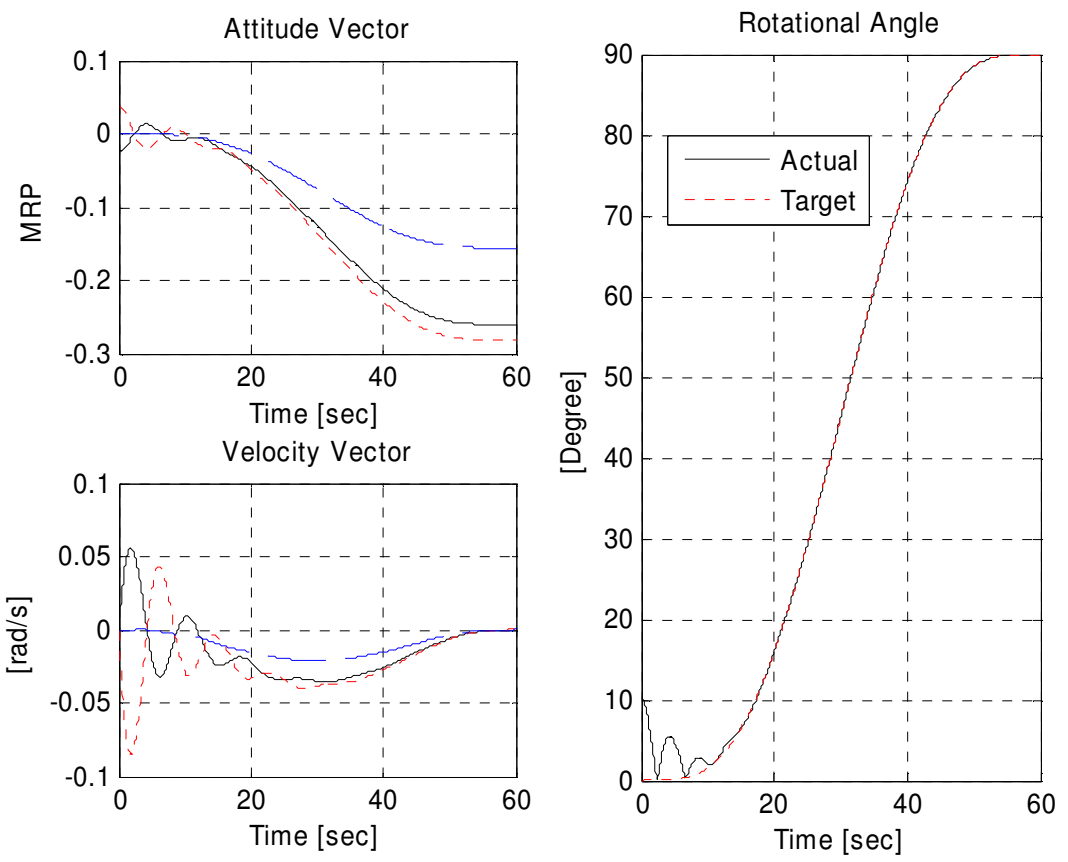

Fig. 7.9 Satellite Motions with Four VSCMGs
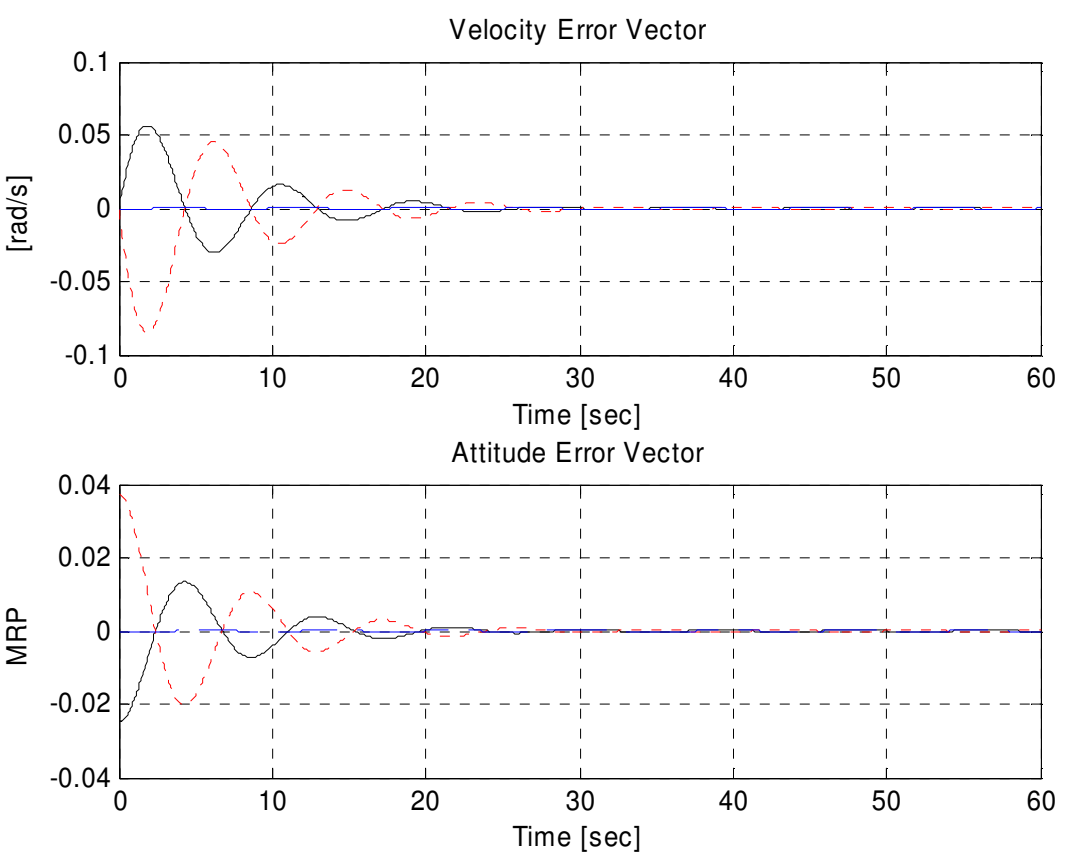

Fig. 7.10 Satellite Error Motions 

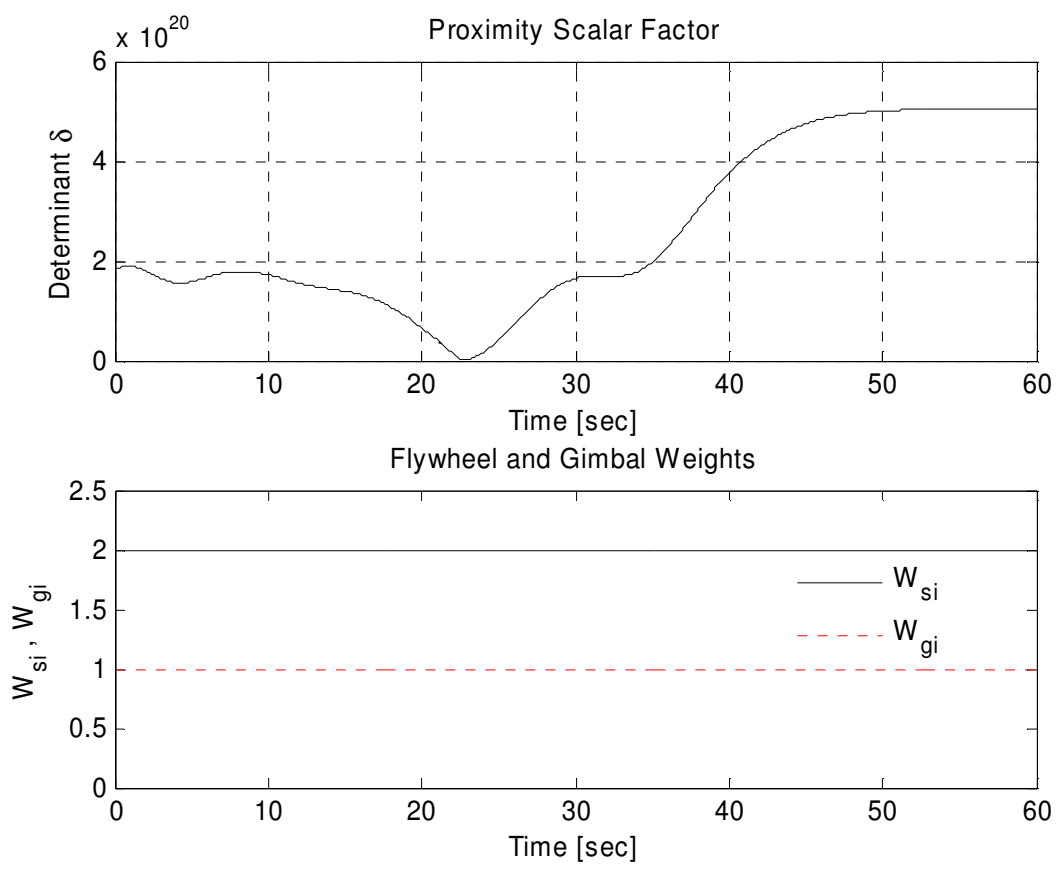

Fig. 7.11 Proximity Scalar and Weights Factor
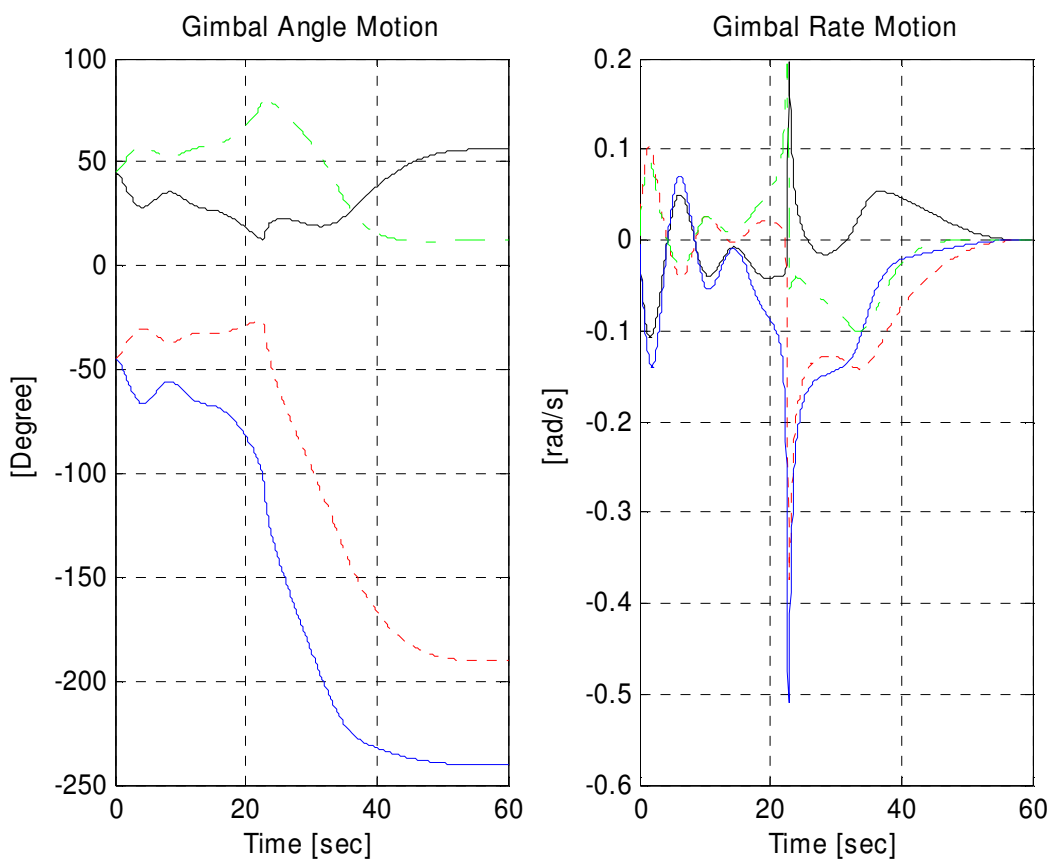

Fig. 7.12 Gimbal Motions 

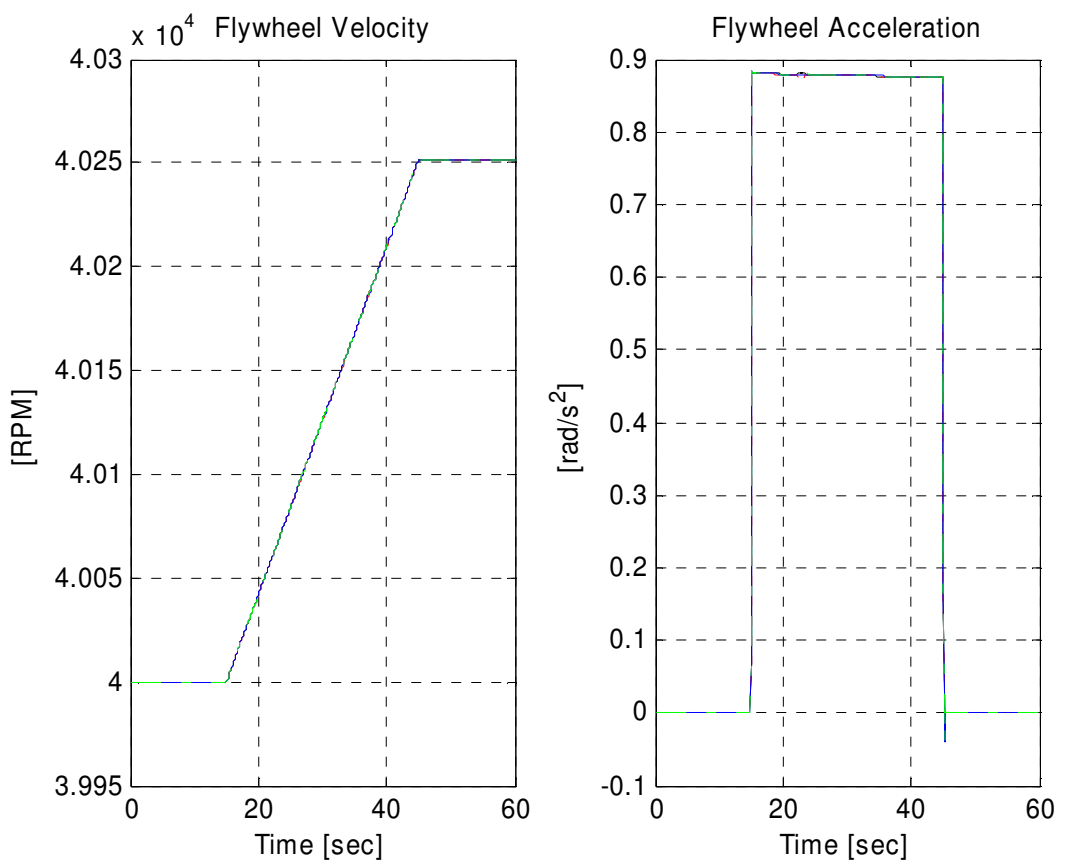

Fig. 7.13 Flywheels (RWs) Motion

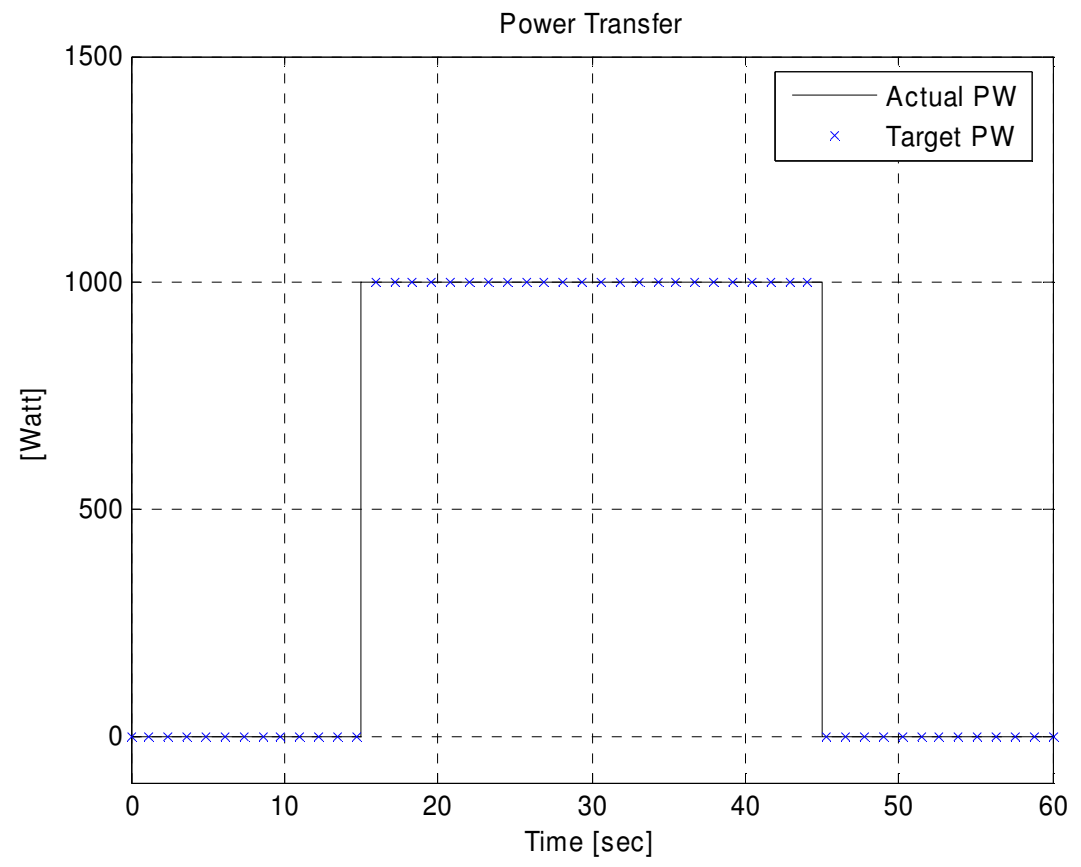

Fig. 7.14 Power Transfer during Attitude Control 


\section{CHAPTER VIII}

\section{CONCLUSION AND FUTURE WORK}

A simulated IPAC operation consisting of a 90 [deg] rotation over 60 [sec], with a $12 \%$ initial attitude error and 500[watt] power transfer for 30[sec] was presented. The IPAC algorithm utilized a nonlinear feedback controller and the magnetic bearings utilized a special gyro torque canceling MIMO control. The magnetic bearing model had a nominal air gap of $(5 \mathrm{e}-4[\mathrm{~m}])$, force limit of $(444.8[\mathrm{~N}])$ and coil voltage limit of (80[volt]). The vibration control masses (VCM) attached to the flexible appendages were very effective for reducing both the power transfer and appendages vibration oscillations. The maximum power ripple is 0.23 [watt] without the VCM at 5\% damping ratio, which is reduced by about $70 \%$ with a $1.35[\mathrm{~kg}] \mathrm{VCM}$. The flexible appendage oscillations also nearly disappear after $25[\mathrm{sec}]$ with the VCM included. The forces transmitted to the satellite were reduced by about $80 \%$ by including a notch filter stage in the MIMO control path. In the case when both the notch and band-pass filters were utilized, the transmitted forces were reduced to $3.5 \mathrm{e}-3[\mathrm{~N}]$.

Two variable speed control moment gyro (VSCMGs) performed attitude control and power tracking functions simultaneously without interfering each other. In the literature, four standard pyramid configuration VSCMGs are utilized for IPAC service, however, this work shows that two VSCMGs can also take care of both attitude and power control functions as same as four VSCMGs case.

For the example considered, the simulation results confirmed the following objectives: 
(1) Demonstrate the effectiveness of IPAC with satellite appendage flexibility, and magnetic bearing feedback dynamics included in the simulation.

(2) Demonstrate the effectiveness of passive dampers for suppressing power oscillations in the IPAC system.

(3) Demonstrate the effectiveness of a MIMO-GYRO torque canceling AMB control algorithm even for a high $\mathrm{I}_{\mathrm{P}} / \mathrm{I}_{\mathrm{T}}$ ratio and when coupled with a IPAC model of a satellite.

(4) Demonstrate the effectiveness of the magnetic bearing suspension to isolate the rotor imbalance forces from the satellite body.

(5) Demonstrate two VSCMGs can be utilized to perform simultaneous attitude control and power tracking functions rather than four VSCMGs case.

Some future work in this area will be seek to demonstrate the effectiveness of IPAC as implemented with only two gimbaled flywheels including MB supported system response with higher energy storage density, nonlinearities of MB system components such as power amplifier saturation and nonlinear MB with magnetic flux saturation and the effects of structural flexibility. Unconditional stability theory of IPAC for MB suspended system and builds and tests in Lab and on satellite. 


\section{REFERENCES}

[1] Tsiotras, P., Shen, H and Hall,C., 2001,“ Satellite Attitude Control for Power Tracking with Energy/Momentum Wheels," AIAA J. Guidance, Control, and Dynamics, 24(1), pp.23-34.

[2] Kim, Y., 2003, "Integrated Power and Attitude Control of a Rigid Satellite with On Board Magnetic Bearing Suspended Rigid Flywheels,” Ph.D. Dissertation, Texas A\&M University, College Station.

[3] Li, Ming-Hsiu., 2004, "Fault Tolerant Control of Homopolar Magnetic Bearing and Circular Sensor Arrays," Ph.D. Dissertation, Texas A\&M University, College Station.

[4] Schaub, H., Vadali, S, R., and Junkins, J, L., 1998, "Feedback Control Law for Variable Speed Control Moment of Gyros," AIAA J. Astronautical Sciences, 46(3), pp.307-328.

[5] Richie, D, J., Tsiotras, P and Fausz, J., 2001, "Simultaneous Attitude Control and Energy Storage using VSCMGs: Theory and Simulation," Proceedings of the American Control Conference, Arlington, Virginia, pp. 3973-3976.

[6] Roes, J. B., 1961, “An Electro-Mechanical Energy Storage System for Space Application," Progress in Astronautics and Rocketry, 3, pp. 613-622.

[7] Sindlinger, R., 1976, "Magnetic Bearing Momentum Wheels with Vernier Gimballing Capability for 3 Axis Active Attitude Control and Energy Storage," Proceedings of the 7th symposium on Automatic Control in Space, Rottach-Egern, West Germany, pp.849-860. 
[8] Brunet, M., 1976, “A New Technology for Three-Axis Stabilized SatelliteActive Magnetic Bearings," Proceedings of the 27th International Astronautical Federation, International Astronautical Congress, Anaheim, California.

[9] Flatley, T. W., 1985, “Tetrahedral Array of Reaction Wheels for Attitude Control and Energy Storage," Proceedings of the 20th Intersociety Energy Conversion Engineering Conference, Warrendale, Pennsylvania, pp.438-443.

[10] Tsiotras, P., 1994, "New Control Laws for the Attitude Stabilization of Rigid Bodies," IFAC Symposium on Automatic Control in Aerospace, Palo Alto, California, pp.316-321.

[11] Schaub, H., Robinett, R. D., and Junkins, J. L., 1996,“ Global Stable Feedback Laws for Near-Minimum-Fuel and Near-Minimum-Time Pointing Maneuvers for a Landmark - Tracking Spacecraft,” AIAA J. Astronautical Sciences, 44(4), pp.443-466.

[12] Okada, Y., Nagai, B., Shimane, T., 1992, "Cross Feedback Stabilization of the Digitally Controlled Magnetic Bearing," ASME J. Vibration and Acoustics, 114, pp. 54-59.

[13] Ahrens, M., Traxler, A., Von Burg, P., and Schweitzer, G., 1994, "Design of a Magnetically Suspended Flywheel Energy Storage Device,” Fourth International Symposium on Magnetic Bearing, ETH, Zurich, pp. 553-558.

[14] Na, U., 1999, "Fault-Tolerant Control of Heteropolar Magnetic Bearings," Ph.D. Dissertation, Texas A\&M University, College Station..

[15] Herzog, R., Buhler, P., and Gahler, C., 1996, "Unbalance Compensation Using 
Generalized Notch Filters in the Multivariable Feedback of Magnetic Bearings," IEEE Trans. Control Systems Technology, 4(5), pp.580-586.

[16] Bhat, P, S., and Bernstein, S, D., 1998, “A Topological Obstruction to Global Asymptotic Stabilization of Rotational Motion and the Unwinding Phenomenon," Proceedings of the American Control Conference, Philadelphia, Pennsylvania, pp. 2785-2789.

[17] Parman, S., Koguch, H., 1999, "Controlling the Attitude Maneuvers of Flexible Spacecraft by Using Time-Optimal/Fuel-Efficient Shaped Inputs,” J. Sound and Vibration, 221(4), pp.545-565.

[18] Kim, Y., Palazzolo, A., Beach, R., and Provenza, A., 2003, “Interaction Dynamics between A Satellite and Onboard Magnetically Suspended Flywheels," $1^{\text {st }}$ International Energy Conversion Engineering Conference. Paper No. AIAA 2003-6109, Portmouth, Virginia.

[19] Ichihara, T., et al., 2005, "Fabrication and Evaluation of Superconducting Magnetic Bearing for 10 KW H-class Flywheel Energy Storage System,” Physica C-Superconductivity and Its Applications, 426, Part 1, pp. 752-758.

[20] Sotelo, G, G., Ferreira, A, C., 2005, "Halbach Array Superconducting Magnetic Bearing for a Flywheel Energy Storage System," IEEE Trans. Applied Superconductivity, 15(2), pp.2253-2256.

[21] Sawada, H., Hashimoto, T., Ninomiya, K., 2001, "High-Stability Attitude Control of Satellite by Magnetic Bearing Wheels," Trans. Japan Society for Aeronautical and Space Sciences, 44(145), pp. 133-141. 
[22] Robinson, A, A., 1982, “A Lightweight, Low-Cost, Magnetic-Bearing Reaction Wheel for Satellite Attitude-Control Applications,” ESA J. European Space Agency, 6(4), pp. 397-406.

[23] Jayaraman, C, P., Kirk, J, A., Anand, D, K., et al., 1991, "Rotor Dynamics of Flywheel Energy Storage Systems,” ASME J. Solar Energy Engineering, 113(1), pp.11-18.

[24] Kirk, J, A., Anand, D, K., 1998, "Satellite Power Using A Magnetically Suspended Flywheel Stack," J. Power Source, 22(3-4), pp. 301-311.

[25] Kirk, J, A., 1997, "Flywheel Energy - Storage. 1. Basic Concepts,” International J. Mechanical Science, 19(4), pp. 223-231.

[26] Kirk, J, A., Studer, P, A., 1997, "Flywheel Energy - Storage.2. Magnetically Suspended Super Flywheel," International J. Mechanical Sciences, 19(4), pp. 223-245.

[27] Kenny, B, H., Kascak, P, E., Jansen, R., Denver, T., Santiago, T., 2005, “Control of a High-Speed Flywheel System for Energy Storage in Space Applications," IEEE Trans. 41(4), pp. 1029-1038.

[28] Christopher, D, A., Beach, R., 1998, "Flywheel Technology Development Program for Aerospace Applications,” IEEE Aerospace and Electronic Systems Magazine, 13(6), pp. 9-14.

[29] Park, J., Palazzolo, A., and Beach, R., 2006, “Integrated Power and Attitude Control (IPAC) of a Satellite via Magnetic Bearing Supported, Flexible Shaft Flywheels," AIAA, Guidance, Navigation and Control Conference and Exhibit, 
Paper No. AIAA 2006-6803, Keystone, Colorado.

[30] Thomson, W, T., 1998, Theory of Vibration with Applications, Fourth Edition, Prentice Hall, Upper Saddle River, New Jersey, pp. 28-29, 188-189.

[31] Junkins, J. L., and Kim, Y., 1993, Introduction to Dynamics and Control of Flexible Structures, AIAA Education Series, Washington D.C., pp.115-119.

[32] Hall, C., Tsiotras, P., and Shen, H., 1998, “Tracking Rigid Body Motion Using Thrusters and Momentum Wheels," AIAA, Paper No. AIAA 98-4471.

[33] Tsiotras, P., 1996, "Stabilization and Optimality Results for the Attitude Control Problem,” AIAA J. Guidance, Control and Dynamics, 19(4), pp.772-779.

[34] Schweitzer, G., Bleuler, H., Traxler, A., 1994, Active Magnetic Bearings, V/D/F, ETH, Zurich, Chap 3.

[35] Meeker, D, C., Maslen, E, H., and Noh, M, D., 1996, “An Augmented Circuit Model for Magnetic Bearings Including Eddy Currents, Fringing, and Leakage," IEEE Trans. Magnetics., 32(4), pp. 3219-3227.

[36] Singiresu S. Rao., 1995, Mechanical Vibrations, Addison-Wesley, Menlo Park, California, Chap 3.6.

[37] Vance, M, J., 1998, Rotordynamics of Turbomachinery, John Wiley \& Sons, New York, Chap 4, pp. 125-130.

[38] Ford, K and Hall, C, D., 1997, "Flexible Spacecraft Reorientations Using Gimbaled Momentum Wheels," AAS/AIAA Astrodynamics Specialist Conference, Sun Valley, Idaho, Paper No. 97-723. 
[39] Oh, H, S., Vadali, S, R., 1991, "Feedback Control and Steering Laws for spacecraft Using Single Gimbal Control Moment of Gyros," AIAA J.

Astronautical Sciences, 39(2), pp.183-203. 


\section{VITA}

Junyoung Park was born in Daegu, South Korea. He grew up in Daegu until his high school year. During his undergraduate year, he served in the military. After he received his Bachelor of Science degree in mechanical engineering from KyungHee University in March 2001, he began his graduate studies in the United States in August 2001. He obtained his Master of Science from the University of Southern California, Los Angeles, and then transferred to Texas A\&M University for the Ph.D. program in mechanical engineering. Mr. Park worked with Dr.Palazzolo in the Vibration Control and Electromechanics Lab since June 2004 until successfully completing his qualifying exams and required course work. During most of this period, he focused on Dynamics, Vibration and Control problems with specific emphasis on magnetically suspended flywheel energy storage systems for satellite and locomotive projects, which were sponsored by NASA and the Texas Transportation of Institute. He has three precious children, Sahn, Sun and Seol, and a lovely wife, Hyeyoung Lee.

Junyoung Park may be contacted at the following address:

Vibration Control and Electromechanics Laboratory

Department of Mechanical Engineering, MS 3123

Texas A\&M University

College Station, Texas, 77843-3123

$979-845-4580$ 